\title{
ALGEBRAIC $K$-THEORY OF RINGS FROM A TOPOLOGICAL VIEWPOINT
}

\author{
Dominique Arlettaz
}

\begin{abstract}
Because of its strong interaction with almost every part of pure mathematics, algebraic $K$-theory has had a spectacular development since its origin in the late fifties. The objective of this paper is to provide the basic definitions of the algebraic $K$-theory of rings and an overview of the main classical theorems. Since the algebraic $K$-groups of a ring $R$ are the homotopy groups of a topological space associated with the general linear group over $R$, it is obvious that many general results follow from arguments from homotopy theory. This paper is essentially devoted to some of them: it explains in particular how methods from stable homotopy theory, group cohomology and Postnikov theory can be used in algebraic $K$-theory.
\end{abstract}

\section{TABle of Contents}

0. Introduction 4

1. The origins of algebraic $K$-theory 5

2. The functors $K_{1}$ and $K_{2} \quad 8$

3. Quillen's higher $K$-groups 16

4. The product structure in algebraic $K$-theory and the $K$-theory spectrum

5. The algebraic $K$-theory of finite fields 30

6. The Hurewicz homomorphism in algebraic $K$-theory 37

7. The Postnikov invariants in algebraic $K$-theory 46

8. The algebraic $K$-theory of number fields and rings of integers 57

9. The algebraic $K$-theory of the ring of integers $\mathbb{Z} \quad 63$

$\begin{array}{ll}\text { 10. Further developments } & 77\end{array}$

$\begin{array}{ll}\text { References } & 78\end{array}$ 


\section{Introduction}

Algebraic $K$-theory is a relatively new mathematical domain which grew up at the end of the fifties on some work by A. Grothendieck on the algebraization of category theory (see Section 1). The category of finitely generated projective modules over a ring $R$ was actually in the center of the preoccupations of the first $K$-theorists because of its relationships with linear groups which play a crucial role in almost all subjects in mathematics. Later, J. Milnor and D. Quillen introduced a very general notion of algebraic $K$-groups $K_{i}(R)$ of any ring $R$ which exhibits some properties of the linear groups over $R$ (see Sections 2 and 3 ). Thus, in some sense, algebraic $K$-theory is a generalization of linear algebra over rings!

The abelian groups $K_{i}(R)$ are homotopy groups of a space which is canonically associated with the general linear group $G L(R)$, i.e., with the group of invertible matrices, over the ring $R$. Therefore, several methods from homotopy theory produce interesting results in algebraic $K$-theory of rings. The objective of the present paper is to describe some of them. This will give us the opportunity to introduce the definition of the groups $K_{i}(R)$ for all integers $i \geq 0$ (in Sections 1, 2 and 3), to explore their structure (in Section 4) and to present classical results (in Sections 5 and 8 ). Moreover, the second part of the paper (Sections 6, $7,8$ and 9$)$ is devoted to more recent results.

Of course, this is far from being a complete list of topological arguments used in algebraic $K$-theory and many other methods also provide very nice results. On the other hand, if, instead of looking at the category of finitely generated projective modules over a ring, we apply the same ideas to other categories, we also get interesting and important developments of algebraic $K$-theory in various directions in mathematics. If the reader wants to get a better and wider understanding of algebraic $K$-theory, he should consult the standard books on this subject (see for example $[\mathbf{1 9}],[\mathbf{2 7}],[\mathbf{2 9}],[\mathbf{5 8}],[\mathbf{6 5}],[\mathbf{7 8}],[\mathbf{8 3}],[\mathbf{9 0}]$; a historical note can be found in $[\mathbf{2 2}])$.

Since our goal is to show how algebraic topology (in particular homotopy theory) can be used in algebraic $K$-theory, we assume that the reader is familiar with the basic notions and results in algebraic topology, homotopy theory and homological algebra (classical references include $[2],[37],[47],[50],[51],[79],[89],[94],[100])$.

Throughout the paper, all maps between topological spaces are supposed to be continuous pointed maps and all rings are rings with units. 


\section{The origins of algebraic $K$-theory}

The very beginning of algebraic $K$-theory is certainly due to some general considerations made by A. Grothendieck. He was motivated by his work in algebraic geometry and introduced the first $K$-theoretical notion in terms of category theory. His idea was to associate to a category $\mathcal{C}$ an abelian group $K(\mathcal{C})$ defined as the free abelian group generated by the isomorphism classes of objects of $\mathcal{C}$ modulo certain relations. Since Grothendieck's mother tongue was German, he chose the letter $K$ for denoting this group of classes ( $K=$ Klassen) of objects of $\mathcal{C}$. This group $K(\mathcal{C})$ was the first algebraic $K$-group: it is now called the Grothendieck group of the category $\mathcal{C}$.

Let us explain in more details the definition of a Grothendieck group by looking at two classical examples. First, let $R$ be a ring and $\mathcal{P}(R)$ the category of finitely generated projective $R$-modules (see $[\mathbf{2 7}$, Sections VII.1 and IX.1], [65, Chapter 1], [78, Section 1.1], [83, Chapter 2], or $[90$, p. 1]).

Definition 1.1. For any ring $R$, the Grothendieck group $K_{0}(R)$ is the quotient of the free abelian group on isomorphism classes $[P]$ of finitely generated projective modules $P \in \mathcal{P}(R)$ by the subgroup generated by the elements of the form $[P \oplus Q]-[P]-[Q]$ for all $P, Q$ in $\mathcal{P}(R)$.

Thus, every element of $K_{0}(R)$ can be written as a difference $[P]-[Q]$ of two generators and one can easily check (see [65, Lemma 1.1], [83, p. 9, Proposition 2], or $[\mathbf{9 0}$, p. 1]) that two generators $[P]$ and $[Q]$ are equal in $K_{0}(R)$ if and only if there is a free $R$-module $R^{s}$ such that $P \oplus R^{s} \cong Q \oplus R^{s}$ (in that case, the $R$-modules $P$ and $Q$ are called stably isomorphic).

A homomorphism of rings $\varphi: R \rightarrow R^{\prime}$ induces a homomorphism of abelian groups

$$
\varphi_{*}: K_{0}(R) \longrightarrow K_{0}\left(R^{\prime}\right)
$$

which is defined as follows. If $P$ is a finitely generated projective $R$-module, there is an $R$-module $Q$ and a positive integer $n$ such that $P \oplus Q \cong$ $R^{n}$ and consequently, $\left(R^{\prime} \otimes_{R} P\right) \oplus\left(R^{\prime} \otimes_{R} Q\right) \cong R^{\prime} \otimes_{R} R^{n} \cong\left(R^{\prime}\right)^{n}$ : in other words $R^{\prime} \otimes_{R} P$ is a finitely generated projective $R^{\prime}$-module. Therefore, let us define for all $[P] \in K_{0}(R) \varphi_{*}([P])=\left[R^{\prime} \otimes_{R} P\right] \in K_{0}\left(R^{\prime}\right)$; $K_{0}(-)$ turns out to be a covariant functor from the category of rings to the category of abelian groups. 
The main problems in the study of the group $K_{0}(R)$ are the following two questions which express the difference between the classical linear algebra over a field and the algebraic $K$-theory which concerns any ring $R$ : is every finitely generated projective module over $R$ a free $R$-module? and is the number of elements in a basis of a free $R$-module an invariant of the module? If both questions would have a positive answer, then the group $K_{0}(R)$ would be infinite cyclic, generated by the class $[R]$ of the free $R$-module of rank 1 . This is of course the case if $R$ is a field, but also for other classes of rings.

Theorem 1.2. If $R$ is a field or a principal domain or a local ring, then $K_{0}(R) \cong \mathbb{Z}$, generated by the class of the free $R$-module of rank 1 .

Proof: See [65, Lemma 1.2], or [78, Sections 1.1 and 1.3].

The main interest of algebraic $K$-theory in dimension 0 is the investigation of projective modules which are not free. For instance, let us look at Dedekind domains, in particular at rings of algebraic integers in number fields.

Theorem 1.3. If $R$ is a Dedekind domain, then $K_{0}(R) \cong \mathbb{Z} \oplus C(R)$, where $C(R)$ denotes the class group of $R$.

Proof: See [65, Chapter 1], or [78, Section 1.4].

The second classical example of a Grothendieck group is given by the topological K-theory which was introduced by A. Grothendieck in 1957 (see [33]) and developed by M. F. Atiyah and F. Hirzebruch (see [20] and [19]). If $X$ is any compact Hausdorff topological space and $\mathbb{F}=\mathbb{R}$ or $\mathbb{C}$, let us denote by $\mathcal{V}(X)$ the category of $\mathbb{F}$-vector bundles over $X$.

Definition 1.4. For any compact Hausdorff topological space $X$, the Grothendieck group $K_{\mathbb{F}}^{0}(X)$ is the quotient of the free abelian group on isomorphism classes $[E]$ of $\mathbb{F}$-vector bundles $E \in \mathcal{V}(X)$ by the subgroup generated by the elements $[E \oplus G]-[E]-[G]$ for all $E, G$ in $\mathcal{V}(X)$, where $\oplus$ is written for the Whitney sum of vector bundles. The abelian group $K_{\mathbb{F}}^{0}(X)$ is called the topological $K$-theory of $X$ (see [19] or Part II of [51] for more details).

Again, every element of $K_{\mathbb{F}}^{0}(X)$ is of the form $[E]-[G]$ where $E$ and $G$ are two vector bundles and $[E]=[G]$ if and only if there is a trivial $\mathbb{F}$-vector bundle $L$ such that $E \oplus L \cong G \oplus L(E$ and $G$ are then called stably equivalent). If $f: X \rightarrow Y$ is a continuous map between two compact Hausdorff topological spaces, and if $E \stackrel{p}{\longrightarrow} Y$ is an $\mathbb{F}$-vector bundle over $Y$, then $f^{*}(E)=\{(x, e) \in X \times E \mid f(x)=p(e)\}$ together 
with $f^{*}(p): f^{*}(E) \rightarrow X$ given by $f^{*}(p)(x, e)=x$ defines an $\mathbb{F}$-vector bundle over $X$. Thus, the map $f$ induces a homomorphism of abelian groups

$$
f^{*}: K_{\mathbb{F}}^{0}(Y) \longrightarrow K_{\mathbb{F}}^{0}(X)
$$

and it turns out that $K_{\mathbb{F}}^{0}(-)$ is a contravariant functor from the category of compact Hausdorff topological spaces to the category of abelian groups.

Remark 1.5. It is not difficult to show that the group $K_{\mathbb{F}}^{0}(X)$ splits as

$$
K_{\mathbb{F}}^{0}(X) \cong \mathbb{Z} \oplus \widetilde{K}_{\mathbb{F}}^{0}(X),
$$

where $\widetilde{K}_{\mathbb{F}}^{0}(X)$, the reduced topological $K$-theory of $X$, is the kernel of the homomorphism $K_{\mathbb{F}}^{0}(X) \rightarrow \mathbb{Z}$ which associates to each vector bundle its rank.

For example, the calculation of $\widetilde{K}_{\mathbb{F}}^{0}(X)$ in the case where $X$ is an $n$-dimensional sphere $S^{n}$ is provided by the celebrated

Theorem 1.6 (Bott periodicity theorem).

(a) $\widetilde{K}_{\mathbb{C}}^{0}\left(S^{n}\right) \cong\left\{\begin{array}{l}\mathbb{Z}, \quad \text { if } n \text { is even, } \\ 0, \quad \text { if } n \text { is odd. }\end{array}\right.$

(b) $\widetilde{K}_{\mathbb{R}}^{0}\left(S^{n}\right) \cong \begin{cases}\mathbb{Z}, & \text { if } n \equiv 0 \bmod 8, \\ \mathbb{Z} / 2, & \text { if } n \equiv 1 \bmod 8, \\ \mathbb{Z} / 2, & \text { if } n \equiv 2 \bmod 8, \\ 0, & \text { if } n \equiv 3 \bmod 8, \\ \mathbb{Z}, & \text { if } n \equiv 4 \bmod 8, \\ 0, & \text { if } n \equiv 5 \bmod 8, \\ 0, & \text { if } n \equiv 6 \bmod 8, \\ 0, & \text { if } n \equiv 7 \bmod 8 .\end{cases}$

Proof: See [35] or [51, p. 109 and Chapter 10]; an alternative proof may be found in $[46]$.

In fact, there is a very strong relationship between topological and algebraic $K$-theory:

Theorem 1.7 (Swan). Let $X$ be any compact Hausdorff topological spa$c e, \mathbb{F}=\mathbb{R}$ or $\mathbb{C}$, and $R(X)$ the ring of continuous functions $X \rightarrow \mathbb{F}$. There is an isomorphism of abelian groups

$$
K_{\mathbb{F}}^{0}(X) \cong K_{0}(R(X)) .
$$


Proof: See [93] or [78, Theorem 1.6.3].

Remark 1.8. It is also possible to describe $K_{0}(R)$ in terms of idempotent matrices over $R$ (see for instance [ $\mathbf{7 8}$, Section 1.2]). This approach is the first sign of the central role played by linear groups in algebraic $K$-theory: it will become especially important for higher $K$-theoretical functors in the next sections.

\section{The functors $K_{1}$ and $K_{2}$}

One of the main objects of interest in linear algebra over a ring $R$ is the general linear group $G L_{n}(R)$ consisting of the multiplicative group of $n \times n$ invertible matrices with coefficients in $R$. In order to look at all invertible matrices of any size in the same group, observe that $G L_{n}(R)$ may be viewed as a subgroup of $G L_{n+1}(R)$ via the upper left inclusion $A \mapsto\left(\begin{array}{cc}A & 0 \\ 0 & 1\end{array}\right)$ and consider the direct limit

$$
G L(R)=\underset{n}{\lim _{\longrightarrow}} G L_{n}(R)=\bigcup_{n=1}^{\infty} G L_{n}(R)
$$

which is called the infinite general linear group. Algebraic $K$-theory is essentially the study of that group for any ring $R$. To begin with, let us investigate the commutator subgroup of $G L(R)$.

Definition 2.1. Let $R$ be any ring, $n$ a positive integer, $i$ and $j$ two integers with $1 \leq i, j \leq n, i \neq j$, and $\lambda$ an element of $R$; let us define the matrix $e_{i, j}^{\lambda}$ to be the $n \times n$ matrix with 1's on the diagonal, $\lambda$ in the $(i, j)$-slot and 0's elsewhere: such a matrix is called an elementary matrix in $G L_{n}(R)$. Let $E_{n}(R)$ denote the subgroup of $G L_{n}(R)$ generated by these matrices and let

$$
E(R)=\underset{n}{\varliminf_{n}} E_{n}(R)=\bigcup_{n=1}^{\infty} E_{n}(R),
$$

where the direct limit is taken via the above upper left inclusions. The group $E(R)$ is called the group of elementary matrices over $R$.

An easy calculation produces the next two lemmas (see [78, Lemma 2.1.2 and Corollary 2.1.3]). 
Lemma 2.2. The elementary matrices over any ring $R$ satisfy the following relations:

(a) $e_{i j}^{\lambda} e_{i j}^{\mu}=e_{i j}^{\lambda+\mu}$,

(b) The commutator $\left[e_{i j}^{\lambda}, e_{k l}^{\mu}\right]=e_{i j}^{\lambda} e_{k l}^{\mu}\left(e_{i j}^{\lambda}\right)^{-1}\left(e_{k l}^{\mu}\right)^{-1}$ satisfies

$$
\left[e_{i j}^{\lambda}, e_{k l}^{\mu}\right]= \begin{cases}1, & \text { if } j \neq k, i \neq l, \\ e_{i l}^{\lambda \mu}, & \text { if } j=k, i \neq l, \\ e_{k j}^{-\mu \lambda}, & \text { if } j \neq k, i=l,\end{cases}
$$

for all $\lambda$ and $\mu$ in $R$. (Notice that there is no simple formula for $\left[e_{i j}^{\lambda}, e_{k l}^{\mu}\right]$ if $j=k$ and $i=l$.)

\section{Lemma 2.3.}

(a) Any triangular matrix with 1's on the diagonal and coefficients in $R$ belongs to the group $E(R)$.

(b) For any matrix $A \in G L_{n}(R)$, the matrix $\left(\begin{array}{cc}A & 0 \\ 0 & A^{-1}\end{array}\right)$ is an element of $E_{2 n}(R)$.

The main property of the group of elementary matrices $E(R)$ is the following.

Lemma 2.4 (Whitehead lemma). For any ring $R$, the commutator subgroups $[G L(R), G L(R)]$ and $[E(R), E(R)]$ are given by

$$
[G L(R), G L(R)]=E(R) \quad \text { and } \quad[E(R), E(R)]=E(R) .
$$

Proof: Because of Lemma 2.2 (b) every generator $e_{i j}^{\lambda}$ of $E(R)$ can be written as the commutator $\left[e_{i j}^{\lambda}, e_{j l}^{1}\right]$. Thus, one gets $[E(R), E(R)]=$ $E(R)$ and the inclusion $E(R) \subset[G L(R), G L(R)]$. In order to prove that $[G L(R), G L(R)] \subset E(R)$, observe that for all matrices $A$ and $B$ in $G L(R)$, one has

$$
\left(\begin{array}{cc}
A B A^{-1} B^{-1} & 0 \\
0 & 1
\end{array}\right)=\left(\begin{array}{cc}
A B & 0 \\
0 & B^{-1} A^{-1}
\end{array}\right)\left(\begin{array}{cc}
A^{-1} & 0 \\
0 & A
\end{array}\right)\left(\begin{array}{cc}
B^{-1} & 0 \\
0 & B
\end{array}\right)
$$

and therefore this matrix belongs to $E_{2 n}(R)$ according to Lemma 2.3 (b).

Remark 2.5. Remember that a group $G$ is called perfect if $G=[G, G]$ or, in other words, if the abelianization $G_{\mathrm{ab}}$ of $G$ is trivial, or, in homological terminology, if $H_{1}(G ; \mathbb{Z})=0$ (see [37, Section II.3], or [50, Section VI.4]). Lemma 2.4 asserts that $E(R)$ is a perfect group for any ring $R$. 
The discovery of that relationship between $G L(R)$ and $E(R)$ was the first step towards the understanding of the linear groups over a ring $R$ : in 1962, it gave rise to the following definition (see [29, Chapter 1], [65, Chapter 3], or [78, Definition 2.1.5]).

Definition 2.6. For any ring $R$, let

$$
K_{1}(R)=G L(R) / E(R)=G L(R)_{\mathrm{ab}} .
$$

A ring homomorphism $f: R \rightarrow R^{\prime}$ induces obviously a homomorphism of abelian groups $f_{*}: K_{1}(R) \rightarrow K_{1}\left(R^{\prime}\right)$ and $K_{1}(-)$ is a covariant functor from the category of rings to the category of abelian groups.

Remember that the abelianization of any group $G$ is isomorphic to its first homology group with integral coefficients $H_{1}(G ; \mathbb{Z})$ (see $[\mathbf{3 7}$, Section II.3] or [50, Section VI.4]).

Corollary 2.7. $K_{1}(R) \cong H_{1}(G L(R) ; \mathbb{Z})$.

If $R$ is commutative, the determinant of square matrices is defined and we may consider the group $S L_{n}(R)$ of $n \times n$ invertible matrices with coefficients in $R$ and determinant +1 , and the infinite special linear group

$$
S L(R)=\underset{n}{\lim } S L_{n}(R)=\bigcup_{n=1}^{\infty} S L_{n}(R) .
$$

This provides the extension of groups

$$
1 \longrightarrow S L(R) \longrightarrow G L(R) \stackrel{\operatorname{det}}{\longrightarrow} R^{\times} \longrightarrow 1,
$$

where $R^{\times}=G L_{1}(R)$ is the group of invertible elements in $R$. Observe that $E(R)$ is clearly a subgroup of $S L(R)$ since any elementary matrix $e_{i, j}^{\lambda}$ has determinant +1 . By looking at the above extension and taking the quotient by $E(R)$, one gets the short exact sequence of abelian groups

$$
1 \longrightarrow S L(R) / E(R) \longrightarrow K_{1}(R) \longrightarrow R^{\times} \longrightarrow 1
$$

which splits since the composition of the inclusion of $R^{\times}=G L_{1}(R)=$ $G L_{1}(R) / E_{1}(R)$ into the group $G L(R) / E(R)=K_{1}(R)$ with the surjection $K_{1}(R) \rightarrow R^{\times}$is the identity. Therefore, we can introduce the functor $S K_{1}(-)$ and obtain the next theorem.

Definition 2.8. For any commutative ring $R$, let

$$
S K_{1}(R)=S L(R) / E(R) .
$$

Theorem 2.9. For any commutative ring $R, K_{1}(R) \cong R^{\times} \oplus S K_{1}(R)$. 
Consequently, it is sufficient to calculate $S K_{1}(R)$ in order to understand $K_{1}(R)$. Let us first mention the following vanishing results.

Theorem 2.10. If $R$ is a field or a commutative local ring or a commutative euclidean ring or the ring of integers in a number field, then $S K_{1}(R)=0$ and the determinant induces an isomorphism

$$
K_{1}(R) \cong R^{\times} .
$$

Proof: See [78, Sections 2.2 and 2.3].

Example 2.11. $K_{1}(\mathbb{Z}) \cong \mathbb{Z} / 2=\{1,-1\}, K_{1}(\mathbb{Z}[i]) \cong \mathbb{Z} / 4=\{1, i,-1,-i\}$, and $K_{1}(F[t]) \cong F^{\times}$for any field $F$.

However, $S K_{1}(R)$ does not vanish for all commutative rings $R$. Here is a result on $K_{1}$ for Dedekind domains.

Definition 2.12. Let $R$ be a commutative ring, and $a$ and $b$ two elements of $R$ such that $R a+R b=R$. If $c$ and $d$ are elements of $R$ such that $a d-b c=1$, then the class of $\left(\begin{array}{ll}a & b \\ c & d\end{array}\right)$ in $S K_{1}(R)$ does not depend on the choice of $c$ and $d$ (see [78, Theorem 2.3.6]): it is denoted by $\left[\begin{array}{l}b \\ a\end{array}\right]$ and called a Mennicke symbol.

If $R$ is a Dedekind domain, it is possible to prove that $K_{1}(R)$ is generated by the image of $G L_{2}(R)$ in $K_{1}(R)=G L(R) / E(R)$ (see [ $\mathbf{7 8}$, Theorem 2.3.5]). This implies the following result.

Theorem 2.13. If $R$ is a Dedekind domain, then the group $S K_{1}(R)$ consists of Mennicke symbols.

Remark 2.14. In that case, $S K_{1}(R)$ is in general non-trivial. However, if $R$ is a Dedekind domain such that $R / \mathfrak{m}$ is a finite field for each nontrivial maximal ideal $\mathfrak{m}$ of $R$, then $S K_{1}(R)$ is a torsion abelian group (see [78, Corollary 2.3.7]).

The next step in the study of the linear groups over a ring $R$ was made by J. Milnor and M. Kervaire in the late sixties when they investigated the universal central extension of the group $E(R)$ (see [65] and [54]).

Definition 2.15. Let $G$ be a group and $A$ an abelian group. A central extension of $G$ by $A$ is a group $H$ together with a surjective homomorphism $\varphi: H \rightarrow G$ such that the kernel of $\varphi$ is isomorphic to $A$ and contained in the center of $H$. A morphism from the central extension $\varphi: H \rightarrow G$ to the central extension $\varphi^{\prime}: H^{\prime} \rightarrow G$ is a group homomorphism $\psi: H \rightarrow H^{\prime}$ such that $\varphi=\varphi^{\prime} \psi$. A central extension $\varphi: H \rightarrow G$ of $G$ is universal if for any central extension $\varphi^{\prime}: H^{\prime} \rightarrow G$ there is a unique morphism of central extensions $\psi: H \rightarrow H^{\prime}$. 
Remark 2.16. This universal property implies clearly that any two universal central extensions of a group $G$ must be isomorphic.

The main result on the existence of universal central extensions is provided by the following theorem.

\section{Theorem 2.17.}

(a) A group $G$ has a universal central extension if and only if $G$ is a perfect group.

(b) In that case, a central extension $\varphi: H \rightarrow G$ of $G$ is universal if and only if $H$ is perfect and all central extensions of $H$ are trivial (i.e., of the form of the projection $A \times H \rightarrow H$ for some abelian group A).

Proof: See [65, Theorems 5.3 and 5.7].

Remark 2.18. In fact, if $G$ is a perfect group presented by $R \longmapsto F \rightarrow G$ (with $F$ a free group), the proof of Theorem 2.17 (a) explicitly constructs a universal central extension $\varphi:[F, F] /[R, F] \rightarrow G$ and it turns out that the kernel of $\varphi$ is $(R \cap[F, F]) /[R, F]$ (see [65, Corollary 5.8]). On the other hand, this corresponds to the Hopf formula for computing the second integral homology group of a group $G$ (see [37, Theorem II.5.3] or [50, Section VI.9]) and we immediately obtain the following consequence: if $\varphi: H \rightarrow G$ is the universal central extension of the perfect group $G$, then

$$
H_{2}(G ; \mathbb{Z}) \cong \operatorname{ker} \varphi
$$

Since the group of elementary matrices $E(R)$ is perfect for any ring $R$ according to Lemma 2.4 , it has a universal extension which can be described as follows.

Definition 2.19. Let $R$ be any ring and $n$ an integer $\geq 3$. The Steinberg group $S t_{n}(R)$ is the free group generated by the elements $x_{i j}^{\lambda}$ for $1 \leq i$, $j \leq n, i \neq j, \lambda \in R$, divided by the relations

(a) $x_{i j}^{\lambda} x_{i j}^{\mu}=x_{i j}^{\lambda+\mu}$,

(b) $\left[x_{i j}^{\lambda}, x_{k l}^{\mu}\right]= \begin{cases}1, & \text { if } j \neq k \text { and } i \neq l, \\ x_{i l}^{\lambda \mu}, & \text { if } j=k \text { and } i \neq l\end{cases}$

Remark 2.20. The relation (a) implies that $\left(x_{i j}^{\lambda}\right)^{-1}=x_{i j}^{-\lambda}$. It then follows from (b) that for $k \neq j, k \neq i$, one has $x_{k i}^{-\mu} x_{i j}^{\lambda} x_{k i}^{\mu} x_{i j}^{-\lambda}=x_{k j}^{-\mu \lambda}$ and consequently $x_{i j}^{\lambda} x_{k i}^{\mu} x_{i j}^{-\lambda} x_{k i}^{-\mu}=x_{k i}^{\mu} x_{k j}^{-\mu \lambda} x_{k i}^{-\mu}=x_{k j}^{-\mu \lambda}$ since $\left[x_{k i}^{\mu}, x_{k j}^{-\mu \lambda}\right]=1$ by (b). In other words, $\left[x_{i j}^{\lambda}, x_{k l}^{\mu}\right]=x_{k j}^{-\mu \lambda}$ if $j \neq k$ and $i=l$. 
Proposition 2.21. For any ring $R$ and any integer $n \geq 3$, the Steinberg group $S t_{n}(R)$ is a perfect group.

Proof: It is obvious that $\left[S t_{n}(R), S t_{n}(R)\right]=S t_{n}(R)$ since every generator $x_{i j}^{\lambda}$ is a commutator by the equality $x_{i j}^{\lambda}=\left[x_{i s}^{\lambda}, x_{s j}^{1}\right]$.

There is clearly a group homomorphism $S t_{n}(R) \rightarrow S t_{n+1}(R)$ sending each generator $x_{i j}^{\lambda}$ of $S t_{n}(R)$ to the corresponding generator $x_{i j}^{\lambda}$ of $S t_{n+1}(R)$ and it is therefore possible to define the infinite Steinberg group $S t(R)=\lim _{n} S t_{n}(R)$.

For any ring $R$ and any integer $n \geq 3$, there is a surjective homomorphism $\varphi: S t_{n}(R) \rightarrow E_{n}(R)$ defined on the generators by $\varphi\left(x_{i j}^{\lambda}\right)=e_{i j}^{\lambda}$ which induces a surjective homomorphism

$$
\varphi: S t(R) \rightarrow E(R) .
$$

The infinite Steinberg group $S t(R)$ plays an important role for the group $E(R)$ because of the following result.

Theorem 2.22. The kernel of $\varphi$ is the center of the group $S t(R)$ and $\varphi: S t(R) \rightarrow E(R)$ is the universal central extension of $E(R)$.

Proof: See [65, Theorem 5.10], or [78, Theorems 4.2.4 and 4.2.7].

This gives rise to the definition of the next $K$-theoretical functor.

Definition 2.23. For any ring $R$, let $K_{2}(R)$ be the kernel of $\varphi: S t(R) \rightarrow$ $E(R)$. Notice that $K_{2}(R)$ is an abelian group which is exactly the center of $S t(R)$.

A ring homomorphism $f: R \rightarrow R^{\prime}$ induces group homomorphisms $S t(R) \rightarrow S t\left(R^{\prime}\right)$ and $E(R) \rightarrow E\left(R^{\prime}\right)$ and consequently a homomorphism of abelian groups $f_{*}: K_{2}(R) \rightarrow K_{2}\left(R^{\prime}\right)$. Thus, $K_{2}(-)$ is a covariant functor from the category of rings to the category of abelian groups.

Remark 2.24. In fact, the relations occuring in the definition of the Steinberg group $S t(R)$ correspond to the obvious relations of the group of elementary matrices $E(R)$. However, $E(R)$ has in general more relations and the group $K_{2}(R)$ measures the non-obvious relations of $E(R)$. From that viewpoint, the knowledge of $K_{2}(R)$ is essential for the understanding of the structure of the group $E(R)$.

Remark 2.18 immediately implies the following homological interpretation of the functor $K_{2}(-)$.

Corollary 2.25. For any ring $R, K_{2}(R) \cong H_{2}(E(R) ; \mathbb{Z})$. 
Remark 2.26. The definitions of $K_{1}(R)$ and $K_{2}(R)$ show the existence of the exact sequence

$$
0 \longrightarrow K_{2}(R) \longrightarrow S t(R) \longrightarrow G L(R) \longrightarrow K_{1}(R) \longrightarrow 0,
$$

where the middle arrow is the composition of the homomorphism $\varphi: S t(R) \rightarrow E(R)$ with the inclusion $E(R) \hookrightarrow G L(R)$.

For the remainder of this section, let us assume that $R$ is a commutative ring. Consider the above homomorphism $\varphi: S t(R) \rightarrow E(R)$. If $x$ and $y$ belong to $E(R)$, one can choose elements $X$ and $Y$ in $S t(R)$ such that $\varphi(X)=x$ and $\varphi(Y)=y$. Of course, $X$ and $Y$ are not unique; however, the commutator $[X, Y]$ is uniquely determined by $x$ and $y$, because for any $a$ and $b$ in $\operatorname{ker} \varphi$ one has $[X a, Y b]=X a Y b a^{-1} X^{-1} b^{-1} Y^{-1}=$ $X Y X^{-1} Y^{-1}=[X, Y]$ since $a$ and $b$ are central. Consequently, we can look at the commutator $[X, Y] \in S t(R)$ and observe that $\varphi([X, Y])=1$ if $x$ and $y$ commute in $E(R)$. Thus, we choose $x=\left(\begin{array}{lll}u & 0 & 0 \\ 0 & u^{-1} & 0 \\ 0 & 0 & 1\end{array}\right)$ and $y=\left(\begin{array}{ccc}v & 0 & 0 \\ 0 & 1 & 0 \\ 0 & 0 & v^{-1}\end{array}\right)$ in $E_{3}(R)$ (see Lemma $2.3(\mathrm{~b})$ ), where $u$ and $v$ are invertible elements in $R$. Since $R$ is commutative, the elements $x$ and $y$ commute and we get an element $[X, Y]$ of $K_{2}(R)$.

Definition 2.27. Let $R$ be a commutative ring. For all $u$ and $v$ in $R^{\times}$, the Steinberg symbol of $u$ and $v$ is the element $\{u, v\}=[X, Y] \in K_{2}(R)$, where $X$ and $Y$ are chosen as above.

Lemma 2.28. For all $u, v$ and $w$ in $R^{\times}$, the Steinberg symbols have the following properties in $K_{2}(R)$ :

(a) $\{u, v\}=\{v, u\}^{-1}$,

(b) $\{u v, w\}=\{u, w\}\{v, w\}$,

(c) $\{u, v w\}=\{u, v\}\{u, w\}$,

(d) $\{u,-u\}=1$,

(e) $\{u, 1-u\}=1$, when $(1-u) \in R^{\times}$.

Proof: See [65, Chapter 9], or [78, Lemma 4.2.14 and Theorem 4.2.17].

Example 2.29. Let $R$ be the ring of integers $\mathbb{Z}$. Then $\mathbb{Z}^{\times}$has only two elements: 1 and -1 . By Lemma 2.28 , it is clear that $\{1,-1\}=$ $\{-1,1\}=\{1,1\}=1$ and that $\{-1,-1\}$ is at most of order 2. In fact, the group $K_{2}(\mathbb{Z})$ is generated by the Steinberg symbol $\{-1,-1\}$ and $K_{2}(\mathbb{Z}) \cong \mathbb{Z} / 2$ (see $[\mathbf{6 5}$, Chapter 10$\left.]\right)$. 
Many results have been obtained on $K_{2}(R)$ in the case where $R$ is a field. The first one asserts that $K_{2}$ of a field is generated by Steinberg symbols and that the above properties (a) and (d) in Lemma 2.28 follow from the other three.

Theorem 2.30 (Matsumoto). If $F$ is a field, then $K_{2}(F)$ is the free abelian group generated by the Steinberg symbols $\{u, v\}$, where $u$ and $v$ run over $F^{\times}$, divided by the relations $\{u v, w\}=\{u, w\}\{v, w\},\{u, v w\}=$ $\{u, v\}\{u, w\},\{u, 1-u\}=1$.

Proof: See [65, Theorem 11.1], or [78, Theorems 4.3.3 and 4.3.15].

If $F$ is a finite field, the situation is much simpler because of the following vanishing result.

Theorem 2.31. If $F$ is a finite field, then all Steinberg symbols in $K_{2}(F)$ are trivial.

Proof: See [65, Corollary 9.9], or [78, Corollary 4.2.18].

We then may deduce a direct consequence of Theorems 2.30 and 2.31.

Corollary 2.32. If $F$ is a finite field, then $K_{2}(F)=0$.

Theorem 2.30 suggests a possible algebraic generalization of the functor $K_{2}(-)$ to higher dimensions: the Milnor $K$-theory which is defined as follows (see $[64]$ ).

Definition 2.33. For any field $F$, the tensor algebra over $F^{\times}$is

$$
T_{*}\left(F^{\times}\right)=\bigoplus_{n=1}^{\infty} T_{n}\left(F^{\times}\right),
$$

where $F^{\times}$is considered as an abelian group and

$$
T_{n}\left(F^{\times}\right)=\underbrace{F^{\times} \otimes_{\mathbb{Z}} F^{\times} \otimes_{\mathbb{Z}} \cdots \otimes_{\mathbb{Z}} F^{\times}}_{n \text { copies }} .
$$

In this algebra, one can consider the ideal $I$ generated by all elements of the form $u \otimes(1-u)$ when $u$ belongs to $F^{\times}$. Then the Milnor K-theory of $F$ is defined by

$$
K_{*}^{M}(F)=T_{*}\left(F^{\times}\right) / I
$$


For each positive integer $n$, the elements of $K_{n}^{M}(F)$ are the symbols $\left\{u_{1}, u_{2}, \ldots, u_{n}\right\}$, with the $u_{i}$ 's in $F^{\times}$satisfying the following rules (additively written):

(a) $\{u,-u\}=0$,

(b) $\{u, 1-u\}=0$,

(c) $\{u, v\}=-\{v, u\}$,

and $K_{*}^{M}(F)$ has an obvious multiplicative structure given by the juxtaposition of symbols.

A lot of work has been done in the study and calculation of Milnor $K$-theory. However, we shall not discuss it here since the purpose of this paper is to study another generalization of $K_{1}$ and $K_{2}$, based on topological considerations: Quillen's higher $K$-theory.

\section{Quillen's higher $K$-groups}

The main ingredient of the notions introduced in Section 2 is the investigation of the linear groups $G L(R), E(R)$ and $S t(R)$. In order to generalize them and to define higher $K$-theoretical functors, the idea presented by D. Quillen in 1970 (see [70]) consists in trying to construct a topological space corresponding in a suitable way to the group $G L(R)$ and studying its homotopical properties. Let us first discuss a very general question on the relationships between topology and group theory.

It is well known that one can associate with any topological space $X$ its fundamental group $\pi_{1}(X)$ and with any discrete group $G$ its classifying space $B G$ which is an Eilenberg-MacLane space $K(G, 1)$. This means that the homotopy groups of $B G$ are all trivial except for $\pi_{1}(B G) \cong G$ and implies that the homology of the group $G$ and the singular homology of the space $B G$ are isomorphic: $H_{*}(G ; A) \cong H_{*}(B G ; A)$ for all coefficients $A$ (see [37, Proposition II.4.1]). Thus, the correspondence $G \rightarrow B G$ and $X \rightarrow \pi_{1}(X)$ fulfills $\pi_{1}(B G) \cong G$, but it is in general not true that the classifying space of the fundamental group of a space $X$ is homotopy equivalent to $X$ : in other words, a topological space $X$ is in general not a $K(G, 1)$ for some group $G$. Therefore, we are forced to introduce a new way to go from group theory to topology. This was essentially done by D. Quillen when he introduced the plus construction (see [70], [59, Section 1.1], [3, Section 3.2], [29, Chapter 5], [48], [78, Section 5.2], or [90, Chapter 2]). 
Theorem 3.1. Let $X$ be a connected $C W$-complex, $P$ a perfect normal subgroup of its fundamental group $\pi_{1}(X)$. There exists a $C W$-complex $X_{P}^{+}$, obtained from $X$ by attaching 2-cells and 3-cells, such that the inclusion $i: X \hookrightarrow X_{P}^{+}$satisfies the following properties:

(a) the induced homomorphism $i_{*}: \pi_{1}(X) \rightarrow \pi_{1}\left(X_{P}^{+}\right)$is exactly the quotient map $\pi_{1}(X) \rightarrow \pi_{1}(X) / P$,

(b) $i$ induces an isomorphism $i_{*}: H_{*}(X ; A) \stackrel{\cong}{\longrightarrow} H_{*}\left(X_{P}^{+} ; A\right)$ for any local coefficient system $A$ on $X_{P}^{+}$,

(c) the space $X_{P}^{+}$is universal in the following sense: if $Y$ is any $C W$-complex and $f: X \rightarrow Y$ any map such that the induced homomorphism $f_{*}: \pi_{1}(X) \rightarrow \pi_{1}(Y)$ fulfils $f_{*}(P)=0$, then there $i s$ a unique map $f^{+}: X_{P}^{+} \rightarrow Y$ such that $f^{+} i=f$. In particular, $X_{P}^{+}$ is unique up to homotopy equivalence.

Proof: The idea of the proof of this theorem is the following. We first attach 2-cells to the $\mathrm{CW}$-complex $X$ in order to kill the subgroup $P$ of $\pi_{1}(X)$; then, we build $X_{P}^{+}$by attaching 3 -cells to the space we just obtained because $X_{P}^{+}$must have the same homology as the original space $X$. Observe that this creates a lot of new elements in the homotopy groups of $X_{P}^{+}$in dimensions $\geq 2$. For details, see [59, Section 1.1], [29, Chapter 5], or [78, Section 5.2].

The universal property of the plus construction implies the following assertion.

Corollary 3.2. Let $X$ and $X^{\prime}$ be two connected $C W$-complexes, $P$ and $P^{\prime}$ two perfect normal subgroups of $\pi_{1}(X)$ and $\pi_{1}\left(X^{\prime}\right)$ respectively and $f: X \rightarrow X^{\prime}$ a map such that $f_{*}(P) \subset P^{\prime}$. Then there is a map $f^{+}: X_{P}^{+} \rightarrow$ $X_{P^{\prime}}^{+}$(unique up to homotopy) making the following diagram commutative:

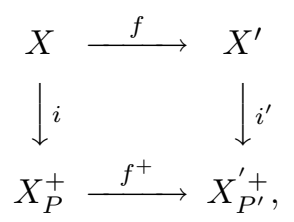

and it is easy to check that the plus construction is functorial.

Let us also mention the following important property. 
Lemma 3.3. If $\widehat{X}$ is the covering space of $X$ associated with the perfect normal subgroup $P$ of $\pi_{1}(X)$, then there is a homotopy equivalence

$$
(\widehat{X})_{P}^{+} \simeq \widetilde{X_{P}^{+}}
$$

between $(\widehat{X})_{P}^{+}$and the universal cover $\widetilde{X_{P}^{+}}$of $X_{P}^{+}$.

Proof: See [59, Proposition 1.1.4] for more details.

Remark 3.4. If $P$ is the maximal perfect normal subgroup of $\pi_{1}(X)$, it is usual to write $X^{+}$for $X_{P}^{+}$.

Let us come back to the question of the correspondence between group theory and topology. If $G$ is a group and $P$ a perfect normal subgroup of $G$, it is indeed a very good idea to look at the space $B G_{P}^{+}$since the celebrated Kan-Thurston theorem asserts that every topological space is of that form (see [52], [61], [28] and [49]).

Theorem 3.5 (Kan-Thurston). For every connected $C W$-complex $X$ there exists a group $G_{X}$ and a map $t_{X}: B G_{X}=K\left(G_{X}, 1\right) \rightarrow X$ which is natural with respect to $X$ and has the following properties:

(a) the homomorphism $\left(t_{X}\right)_{*}: \pi_{1}\left(B G_{X}\right) \cong G_{X} \rightarrow \pi_{1}(X)$ induced by $t_{X}$ is surjective,

(b) the map $t_{X}$ induces an isomorphism $\left(t_{X}\right)_{*}: H_{*}\left(B G_{X} ; A\right) \stackrel{\cong}{\longrightarrow}$ $H_{*}(X ; A)$ for any local coefficient system $A$ on $X$.

This implies the following consequences.

Corollary 3.6. For every connected $C W$-complex $X$, the kernel $P_{X}$ of $\left(t_{X}\right)_{*}: G_{X} \rightarrow \pi_{1}(X)$ is perfect.

Proof: Let us look at the pull-back $Y$ of the diagram

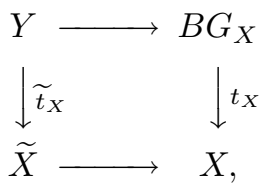

in which $\tilde{X}$ is the universal cover of $X$. Both horizontal maps have the same homotopy fiber $\pi_{1}(X)$ and $t_{X}$ induces an isomorphism on homology with any local coefficient system. Therefore, the comparison theorem for the Serre spectral sequences of both horizontal maps implies that $\left(\widetilde{t}_{X}\right)_{*}: H_{1}(Y ; \mathbb{Z}) \rightarrow H_{1}(\tilde{X} ; \mathbb{Z})$ is an isomorphism and that $H_{1}(Y ; \mathbb{Z})$ vanishes since $H_{1}(\widetilde{X} ; \mathbb{Z})=0$. The homotopy exact sequence of the fibration

$$
Y \longrightarrow B G_{X} \longrightarrow B \pi_{1}(X)
$$


shows that $\pi_{1}(Y) \cong P_{X}$. Consequently, $\left(P_{X}\right)_{a b} \cong \pi_{1}(Y)_{a b} \cong H_{1}(Y ; \mathbb{Z})=$ 0 , in other words, $P_{X}$ is a perfect group.

Theorem 3.7. For every connected $C W$-complex $X$, there exists a group $G_{X}$ together with a perfect normal subgroup $P_{X}$ such that one has a homotopy equivalence $\left(B G_{X}\right)_{P_{X}}^{+} \simeq X$.

Proof: For a connected CW-complex $X$, let us consider the group $G_{X}$, the map $t_{X}: B G_{X} \rightarrow X$ and the perfect group $P_{X}=\operatorname{ker}\left(\left(t_{X}\right)_{*}: G_{X} \rightarrow\right.$ $\left.\pi_{1}(X)\right)$ given by Theorem 3.5 and Corollary 3.6. Then, consider the plus construction $\left(B G_{X}\right)_{P_{X}}^{+}$and apply Theorem 3.1 (c): there is a map $t_{X}^{+}:\left(B G_{X}\right)_{P_{X}}^{+} \rightarrow X$ which induces an isomorphism on $\pi_{1}$ and on all homology groups. The generalized Whitehead theorem (see [48, Corollary 1.5], or $\left[\mathbf{2 9}\right.$, Proposition 4.15]) finally implies that $t_{X}^{+}$is a homotopy equivalence.

Definition 3.8 (see [49, Sections 1 and 2]). A topogenic group is a pair $(G, P)$, where $G$ is a group and $P$ a perfect normal subgroup of $G$. In particular, a perfect group $P$ can be viewed as a topogenic group because of the pair $(P, P)$. A morphism of topogenic groups $f:(G, P) \rightarrow\left(G^{\prime}, P^{\prime}\right)$ is a group homomorphism $f: G \rightarrow G^{\prime}$ such that $f(P) \subset P^{\prime}$. An equivalence of topogenic groups is a morphism $f:(G, P) \rightarrow\left(G^{\prime}, P^{\prime}\right)$ inducing an isomorphism $G / P \stackrel{\cong}{\longrightarrow} G^{\prime} / P^{\prime}$ and an isomorphism on homology $f_{*}: H_{*}(G ; A) \stackrel{\cong}{\longrightarrow} H_{*}\left(G^{\prime} ; A\right)$ for all coefficients $A$. Consequently, if two topogenic groups are equivalent, there is a map $B f^{+}: B G_{P}^{+} \rightarrow B G_{P^{\prime}}^{+}$ between the corresponding $\mathrm{CW}$-complexes which induces an isomorphism on the fundamental group and on all homology groups. Again, it follows from the generalized Whitehead theorem that $B f^{+}: B G_{P}^{+} \rightarrow$ $B G_{P^{\prime}}^{\prime+}$ is a homotopy equivalence.

Consequently, this establishes a very nice one-to-one correspondence between group theory and topology (see [28, Section 11], and [49, Section 2]):

$$
\begin{array}{ccc}
\begin{array}{c}
\text { equivalence classes } \\
\text { of topogenic groups }
\end{array} & \longleftrightarrow & \begin{array}{c}
\text { homotopy types } \\
\text { of CW-complexes }
\end{array} \\
(G, P) & \longrightarrow & B G_{P}^{+} \\
\left(G_{X}, P_{X}\right) & \longleftarrow & X .
\end{array}
$$

Remark 3.9. By Theorem 3.1 the space $B G_{P}^{+}$associated with the topogenic group $(G, P)$ satisfies the following properties:

(a) $\pi_{1}\left(B G_{P}^{+}\right) \cong G / P$,

(b) $H_{i}\left(B G_{P}^{+} ; A\right) \cong H_{i}(G ; A)$ for any coefficients $A$. 
Example 3.10. The perfect groups correspond to the simply connected CW-complexes, because for any perfect group $P$, the space $B P_{P}^{+}$associated with the topogenic group $(P, P)$ has trivial fundamental group $\pi_{1} B P_{P}^{+} \cong P / P=0$.

Example 3.11. Let $\Sigma_{\infty}=\lim _{n} \Sigma_{n}$ be the infinite symmetric group and $A_{\infty}=\lim _{n} A_{n}$ the infinite alternating group which is perfect. The Barratt-Priddy theorem [26] asserts that the topogenic group $\left(\Sigma_{\infty}, A_{\infty}\right)$ corresponds to $\left(B \Sigma_{\infty}\right)_{A_{\infty}}^{+}$which is homotopy equivalent to the connected component of the space $Q_{0} S^{0}=\varliminf_{n} \Omega^{n} S^{n}$ whose homotopy groups are the stable homotopy groups of spheres $\pi_{i}\left(Q_{0} S^{0}\right)=\varliminf_{n} \pi_{i}\left(\Omega^{n} S^{n}\right)=$ $\lim _{n} \pi_{i+n}\left(S^{n}\right), i \geq 0$.

In order to define a suitable generalization of the functor $K_{1}(-)$ and $K_{2}(-)$ for rings, let us consider again the infinite general linear group $G L(R)$ with coefficients in a ring $R$ and its perfect normal subgroup generated by elementary matrices $E(R)$ : we get the topogenic group $(G L(R), E(R))$. The higher algebraic $K$-theory of $R$ is the study of the corresponding topological space $B G L(R)_{E(R)}^{+}$(for simplicity, we shall write $B G L(R)^{+}$for $B G L(R)_{E(R)}^{+}$according to Remark 3.4).

Definition 3.12 (Quillen (see [70])). For any ring $R$ and any positive integer $i$, the $i$-th algebraic $K$-theory group of $R$ is

$$
K_{i}(R)=\pi_{i}\left(B G L(R)^{+}\right) .
$$

Let us check that this definition extends the definition of $K_{1}(R)$ and $K_{2}(R)$ given in Section 2. Remark 3.9 (a) shows that $\pi_{1}\left(B G L(R)^{+}\right) \cong$ $G L(R) / E(R)$ and this group is exactly $K_{1}(R)$ according to Definition 2.6.

Notice also that Lemma 3.3 shows that the universal cover of $B G L(R)^{+}$is the space $B E(R)^{+}$associated with the topogenic group $(E(R), E(R))$. Therefore, we get the following result.

Theorem 3.13. For any ring $R$ the space $B E(R)^{+}$is simply connected and for all integers $i \geq 2$,

$$
K_{i}(R) \cong \pi_{i}(B E(R))^{+} .
$$

In particular, the group $K_{2}(R)$ given by Definition 3.12 coincides with the group $\pi_{2}\left(B E(R)^{+}\right)$which is isomorphic to $H_{2}\left(B E(R)^{+} ; \mathbb{Z}\right) \cong$ $H_{2}(E(R) ; \mathbb{Z})$ (see Remark 3.9 (b)) because of the Hurewicz theorem. Corollary 2.25 then implies that it is isomorphic to $K_{2}(R)$ as defined in Definition 2.23. 
Of course, a ring homomorphism $f: R \rightarrow R^{\prime}$ induces a group homomorphism $G L(R) \rightarrow G L\left(R^{\prime}\right)$ whose restriction to $E(R)$ sends $E(R)$ into $E\left(R^{\prime}\right)$. Thus, Corollary 3.2 implies the existence of a map $B G L(R)^{+} \rightarrow$ $B G L\left(R^{\prime}\right)^{+}$and consequently of a homomorphism of abelian groups $f_{*}: K_{i}(R) \rightarrow K_{i}\left(R^{\prime}\right)$ for all $i \geq 1$. Moreover, one can check that $K_{n}(-)$ is a covariant functor from the category of rings to the category of abelian groups.

As we just observed, one can express the first two $K$-groups homologically (see Corollaries 2.7 and 2.25):

$$
\begin{aligned}
& K_{1}(R) \cong H_{1}(G L(R) ; \mathbb{Z}), \\
& K_{2}(R) \cong H_{2}(E(R) ; \mathbb{Z}) .
\end{aligned}
$$

In the same way, we can prove the following result.

Theorem 3.14. Let $S t(R)$ be the infinite Steinberg group over a ring $R$. Then

(a) the space $B S t(R)^{+}$is 2-connected,

(b) $K_{i}(R) \cong \pi_{i}\left(B S t(R)^{+}\right)$for all $i \geq 3$,

(c) $K_{3}(R) \cong H_{3}(S t(R) ; \mathbb{Z})$.

Proof: Let us consider the universal central extension

$$
0 \longrightarrow K_{2}(R) \longrightarrow S t(R) \stackrel{\varphi}{\longrightarrow} E(R) \longrightarrow 1
$$

and the associated fibration of classifying spaces

$$
B K_{2}(R) \longrightarrow B S t(R) \stackrel{B \varphi}{\longrightarrow} B E(R) .
$$

Since $E(R)$ and $S t(R)$ are perfect, one can perform the + construction to both spaces $B S t(R)$ and $B E(R)$. If one denotes by $F$ the homotopy fiber of the induced map

$$
B S t(R)^{+} \stackrel{B \varphi^{+}}{\longrightarrow} B E(R)^{+}
$$

one has the following commutative diagram where both rows are fibrations:

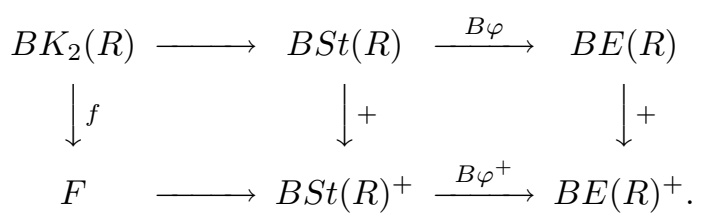

Since $K_{2}(R)$ is the center of $S t(R)$ by Theorem 2.22, the action of $\pi_{1}(B E(R))$ on the homology of $B K_{2}(R)$ is trivial. The same holds for the second fibration since $B E(R)^{+}$is simply connected. The two right vertical arrows induce isomorphisms on integral homology by Theorem 3.1. 
Therefore, the comparison theorem for spectral sequences implies that $f_{*}: H_{*}\left(B K_{2}(R) ; \mathbb{Z}\right) \rightarrow H_{*}(F ; \mathbb{Z})$ is an isomorphism. Since $S t(R)$ is perfect, the space $B S t(R)^{+}$is also simply connected. On the other hand, it is known that $H_{2}\left(B S t(R)^{+} ; \mathbb{Z}\right) \cong H_{2}(S t(R) ; \mathbb{Z})=0$ according to [54]. Consequently, the Hurewicz theorem shows that $B S t(R)^{+}$is actually 2 -connected. Now, let us look at the homotopy exact sequence

$$
\underbrace{\pi_{2}\left(B S t(R)^{+}\right)}_{=0} \longrightarrow \underbrace{\pi_{2}\left(B E(R)^{+}\right)}_{\cong K_{2}(R)} \stackrel{B \varphi_{*}^{+}}{\longrightarrow} \pi_{1}(F) \longrightarrow 0
$$

of the fibration

$$
F \longrightarrow B S t(R)^{+} \longrightarrow B E(R)^{+} .
$$

Since $\pi_{1}(F) \cong K_{2}(R)$, it is an abelian group. Consequently, the Hurewicz homomorphism $\pi_{1}(F) \rightarrow H_{1}(F ; \mathbb{Z})$ is an isomorphism. Then, consider the commutative diagram

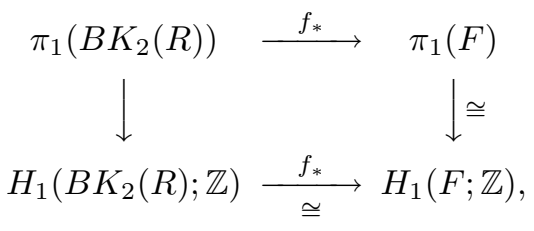

where the vertical arrows are Hurewicz homomorphisms. The left vertical arrow is an isomorphism since $B K_{2}(R)$ is an Eilenberg-MacLane space $K\left(K_{2}(R), 1\right)$ with $K_{2}(R)$ abelian. Thus, $f_{*}: \pi_{1}\left(B K_{2}(R)\right) \rightarrow \pi_{1}(F)$ is an isomorphism and $f: B K_{2}(R) \rightarrow F$ is a homotopy equivalence because of the generalized Whitehead theorem (see [48, Corollary 1.5], or [29, Proposition 4.15]). In other words, one obtains the following fibration (which can also be deduced from a more general topological argument, see [29, Theorem 6.4], [59, Théorème 1.3.5], or [96, Lemma 3.1]):

$$
B K_{2}(R) \longrightarrow B S t(R)^{+} \longrightarrow B E(R)^{+} \text {. }
$$

The homotopy exact sequence of that fibration shows that

$$
\pi_{i}\left(B S t(R)^{+}\right) \underset{B \varphi_{*}^{+}}{\stackrel{\cong}{\longrightarrow}} \pi_{i}\left(B E(R)^{+}\right) \cong K_{i}(R)
$$

for all $i \geq 3$ and that $B S t(R)^{+}$is the 2-connected cover of $B G L(R)^{+}$. Finally, it follows from the Hurewicz theorem that

$$
K_{3}(R) \cong \pi_{3}\left(B S t(R)^{+}\right) \cong H_{3}\left(B S t(R)^{+} ; \mathbb{Z}\right) \cong H_{3}(S t(R) ; \mathbb{Z}) .
$$

Remark 3.15. This homological interpretation of the groups $K_{i}(R)$ for $i=1,2,3$, suggests the following generalization. Let us denote by $B G L(R)^{+}(m)$ the $m$-connected cover of the space $B G L(R)^{+}$for 
$m \geq 0 ;$ more precisely, $B G L(R)^{+}(m)$ is $m$-connected and there is a map $B G L(R)^{+}(m) \rightarrow B G L(R)^{+}$inducing an isomorphism on $\pi_{i}$ for $i \geq m+1$. For instance, $B G L(R)^{+}(1)=B E(R)^{+}$and $B G L(R)^{+}(2)=$ $B S t(R)^{+}$. According to the Kan-Thurston theorem (see Theorem 3.5), there exists a perfect group $G_{m}(R)$ for each positive integer $m$ such that $B G_{m}(R)^{+} \simeq B G L(R)^{+}(m)$. Consequently,

$$
K_{i}(R) \cong \pi_{i}\left(B G L(R)^{+}(m)\right) \cong \pi_{i}\left(B G_{m}(R)^{+}\right)
$$

for $i \geq m+1$ and

$$
\begin{aligned}
K_{m+1}(R) \cong \pi_{m+1}\left(B G_{m}(R)^{+}\right) & \cong H_{m+1}\left(B G_{m}(R)^{+} ; \mathbb{Z}\right) \\
& \cong H_{m+1}\left(G_{m}(R) ; \mathbb{Z}\right)
\end{aligned}
$$

since $B G_{m}(R)^{+}$is $m$-connected. Thus, there exists a list of groups $G_{0}(R)=G L(R), G_{1}(R)=E(R), G_{2}(R)=S t(R), G_{3}(R), G_{4}(R), \ldots$ whose homology represents the $K$-groups of $R$. Unfortunately, we do not have any explicit description of the groups $G_{m}(R)$ for $m \geq 3$.

Remark 3.16. D. Quillen also gave another equivalent definition of the higher $K$-groups. Let $R$ be a ring and consider again the category $\mathcal{P}(R)$ of finitely generated projective $R$-modules. He constructed a new category $Q \mathcal{P}(R)$ and its classifying space $B Q \mathcal{P}(R)$. Furthermore, he defined

$$
K_{i}(R)=\pi_{i+1}(B Q \mathcal{P}(R))
$$

for $i \geq 0$. In fact, it turns out that the loop space $\Omega B Q \mathcal{P}(R)$ of $B Q \mathcal{P}(R)$ satisfies

$$
\Omega B Q \mathcal{P}(R) \simeq K_{0}(R) \times B G L(R)^{+}
$$

for any ring $R$ (see [72, Sections 1 and 2], or [74, Theorem 1], for the details of that construction). Of course, Quillen proved that both definitions of the $K$-groups coincide.

In the present paper we want to concentrate our attention on the first definition of the $K$-groups (see Definition 3.12). The higher algebraic $K$-theory of a ring $R$ is really the study of the space $B G L(R)^{+}$whose homotopy type is determined by its homotopy groups $K_{i}(R)$ and by its Postnikov $k$-invariants (see Section 7 ). The space $B G L(R)^{+}$has actually many other interesting properties. The remainder of the paper is devoted to the investigation of some of them. 


\section{The product structure in algebraic $K$-theory and the $K$-theory spectrum}

The goal of this section is to show that the algebraic $K$-theory space $B G L(R)^{+}$of any ring $R$ has actually a very rich structure. Let us start by considering a ring $R$ and the homomorphism

$$
\oplus: G L(R) \times G L(R) \longrightarrow G L(R)
$$

given by

$$
(\alpha \oplus \beta)_{i j}= \begin{cases}\alpha_{k l}, & \text { if } i=2 k-1 \text { and } j=2 l-1, \\ \beta_{k l}, & \text { if } i=2 k \text { and } j=2 l, \\ 0, & \text { otherwise, }\end{cases}
$$

for $\alpha, \beta \in G L(R)$. Since there is a homotopy equivalence $B G L(R)^{+} \times$ $B G L(R)^{+} \simeq B(G L(R) \times G L(R))^{+}$(see [59, Proposition 1.1.4]), we can define the map

$$
\mu: B G L(R)^{+} \times B G L(R)^{+} \simeq B(G L(R) \times G L(R))^{+} \stackrel{\oplus^{+}}{\longrightarrow} B G L(R)^{+}
$$

which endows $B G L(R)^{+}$with the following structure.

Proposition 4.1. For any ring $R$, the space $B G L(R)^{+}$, together with the map $\mu$, is a commutative $H$-group.

Proof: See [59, Théorème 1.2.6].

Now, let $R$ and $R^{\prime}$ be two rings and let us denote by $R \otimes R^{\prime}$ the tensor product $R \otimes_{\mathbb{Z}} R^{\prime}$ over $\mathbb{Z}$. The tensor product of matrices induces a homomorphism

$$
G L_{m}(R) \times G L_{n}\left(R^{\prime}\right) \longrightarrow G L_{m n}\left(R \otimes R^{\prime}\right)
$$

and a map

$$
\widetilde{\eta}_{m, n}^{R, R^{\prime}}: B G L_{m}(R)^{+} \times B G L_{n}\left(R^{\prime}\right)^{+} \longrightarrow B G L_{m n}\left(R \otimes R^{\prime}\right)^{+} .
$$

By composing this map with the map induced by the upper left inclusion $G L_{m n}\left(R \otimes R^{\prime}\right) \hookrightarrow G L\left(R \otimes R^{\prime}\right)$ we get a map

$$
\eta_{m, n}^{R, R^{\prime}}: B G L_{m}(R)^{+} \times B G L_{n}\left(R^{\prime}\right)^{+} \longrightarrow B G L\left(R \otimes R^{\prime}\right)^{+} .
$$

Then, let us define the map

$$
\gamma_{m, n}^{R, R^{\prime}}: B G L_{m}(R)^{+} \times B G L_{n}\left(R^{\prime}\right)^{+} \longrightarrow B G L\left(R \otimes R^{\prime}\right)^{+}
$$

by $\gamma_{m, n}^{R, R^{\prime}}(x, y)=\eta_{m, n}^{R, R^{\prime}}(x, y)-\eta_{m, n}^{R, R^{\prime}}\left(x_{0}, y\right)-\eta_{m, n}^{R, R^{\prime}}\left(x, y_{0}\right)$, where $x_{0}$ and $y_{0}$ are the base points of $B G L_{m}(R)^{+}$and $B G L_{n}\left(R^{\prime}\right)^{+}$respectively, and where "-" is the subtraction in the sense of the $H$-space structure of 
the space $B G L\left(R \otimes R^{\prime}\right)^{+}$. Since the maps $\gamma_{m, n}^{R, R^{\prime}}$ are compatible (up to homotopy) with the stabilizations $i_{m, n}^{R, R^{\prime}}: B G L_{m}(R)^{+} \times B G L_{n}\left(R^{\prime}\right)^{+} \rightarrow$ $B G L_{m+1}(R)^{+} \times B G L_{n+1}\left(R^{\prime}\right)^{+}$induced by upper left inclusions, i.e., $\gamma_{m, n}^{R, R^{\prime}} \simeq \gamma_{m+1, n+1}^{R, R^{\prime}} i_{m, n}^{R, R^{\prime}},($ see [59, Lemme 2.1.3]), we get a map

$$
\gamma^{R, R^{\prime}}: B G L(R)^{+} \times B G L\left(R^{\prime}\right)^{+} \longrightarrow B G L\left(R \otimes R^{\prime}\right)^{+},
$$

which is unique up to weak homotopy (see [59, Lemme 2.1.6 and Remarque 2.1.9]). By definition, this map $\gamma^{R, R^{\prime}}$ is homotopic to the trivial map on the wedge $B G L(R)^{+} \vee B G L\left(R^{\prime}\right)^{+}$. Consequently, it finally induces a map

$$
\widehat{\gamma}^{R, R^{\prime}}: B G L(R)^{+} \wedge B G L\left(R^{\prime}\right)^{+} \longrightarrow B G L\left(R \otimes R^{\prime}\right)^{+} .
$$

It turns out that this map $\widehat{\gamma}^{R, R^{\prime}}$ is natural in $R$ and $R^{\prime}$, bilinear, associative and commutative, up to weak homotopy (see [59, Proposition 2.1.8]). It enables us to give the following definition (see [59, Définition 2.1.10]; an alternative definition can be found in Chapter 13 of $[29])$.

Definition 4.2 (Loday). For all rings $R$ and $R^{\prime}$, and for all integers $i, j \geq 1$, the product map

$$
\begin{aligned}
\star: K_{i}(R) \times K_{j}\left(R^{\prime}\right)= & \pi_{i}\left(B G L(R)^{+}\right) \times \pi_{j}\left(B G L\left(R^{\prime}\right)^{+}\right) \\
& \longrightarrow \pi_{i+j}\left(B G L\left(R \otimes R^{\prime}\right)^{+}\right)=K_{i+j}\left(R \otimes R^{\prime}\right),
\end{aligned}
$$

is defined as follows: if $x \in K_{i}(R)$ and $y \in K_{j}\left(R^{\prime}\right)$ are represented by $\alpha: S^{i} \rightarrow B G L(R)^{+}$and $\beta: S^{j} \rightarrow B G L\left(R^{\prime}\right)^{+}$respectively, then

$$
\begin{aligned}
& x \star y=\left[S^{i+j} \simeq S^{i} \wedge S^{j} \stackrel{\alpha \wedge \beta}{\longrightarrow} B G L(R)^{+} \wedge B G L\left(R^{\prime}\right)^{+}\right. \\
&\left.\stackrel{\widehat{\gamma}^{R, R^{\prime}}}{\longrightarrow} B G L\left(R \otimes R^{\prime}\right)^{+}\right] .
\end{aligned}
$$

One can then immediately deduce the following properties (see [59, Théorème 2.1.11]).

Proposition 4.3. The product map $\star: K_{i}(R) \times K_{j}\left(R^{\prime}\right) \rightarrow K_{i+j}\left(R \otimes R^{\prime}\right)$ is natural in $R$ and $R^{\prime}$, bilinear and associative for all $i, j \geq 1$.

Remark 4.4. Because of that proposition, we can consider the above product on the tensor product $K_{i}(R) \otimes K_{j}\left(R^{\prime}\right)$ and we shall also denote it by the symbol $\star$ :

$$
\star: K_{i}(R) \otimes K_{j}\left(R^{\prime}\right) \longrightarrow K_{i+j}\left(R \otimes R^{\prime}\right) .
$$


Now, let us look at the special case where $R^{\prime}=R$. If $R$ is a commutative ring, the ring homomorphism $\nabla: R \otimes R \rightarrow R$ given by $\nabla(a \otimes b)=a b$ induces a ring structure on $K_{*}(R)$.

Definition 4.5. If $R$ is a commutative ring, then there is a product map (also denoted by $\star$ )

$$
\star: K_{i}(R) \otimes K_{j}(R) \stackrel{\star}{\longrightarrow} K_{i+j}(R \otimes R) \stackrel{\nabla_{*}}{\longrightarrow} K_{i+j}(R)
$$

for all $i, j \geq 1$.

This product satisfies:

Proposition 4.6. If $R$ is a commutative ring, then for all $x \in K_{i}(R)$ and $y \in K_{j}(R)$ with $i, j \geq 1$, one has $x \star y=(-1)^{i j} y \star x$.

Proof: Let again $x \in K_{i}(R)$ and $y \in K_{j}(R)$ be represented by $\alpha: S^{i} \rightarrow$ $B G L(R)^{+}$and $\beta: S^{j} \rightarrow B G L(R)^{+}$respectively. Let $t: R \otimes R \rightarrow R \otimes R$ denote the homomorphism given by $t(a \otimes b)=b \otimes a$ and $s: S^{i} \wedge S^{j} \rightarrow$ $S^{j} \wedge S^{i}$ the homeomorphism which exchanges the factors. Since $R$ is commutative, $\nabla t=\nabla$ and we get the commutative diagram

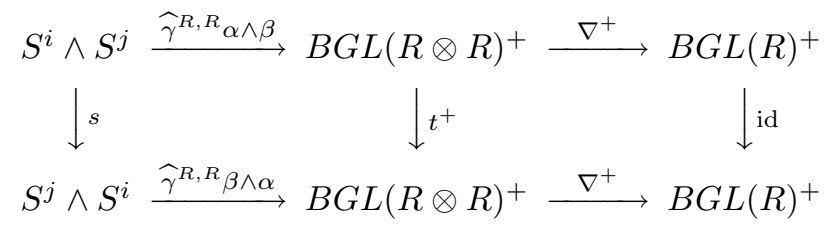

which provides the result since the homotopy class of $s$ is $(-1)^{i j} \in$ $\pi_{i+j}\left(S^{i+j}\right) \cong \mathbb{Z}$.

The remainder of this section is devoted to further investigation of the $H$-space structure of the space $B G L(R)^{+}$(see $[\mathbf{5 9}$, Sections 1.4 and $2.3]$ ). Let us first consider the ring of integers $R=\mathbb{Z}$. Its cone $C \mathbb{Z}$ is the set of all infinite matrices with integral coefficients having only a finite number of non-trivial elements on each row and on each column. This set turns out to be a ring by the usual addition and multiplication of matrices. Let $J \mathbb{Z}$ be the ideal of $C \mathbb{Z}$ which consists of all matrices having only finitely many non-trivial coefficients. Finally, let us define the suspension of $\mathbb{Z}$ to be the quotient $\operatorname{ring} \Sigma \mathbb{Z}=C \mathbb{Z} / J \mathbb{Z}$.

Definition 4.7. For any ring $R$, the suspension of $R$ is the ring

$$
\Sigma R=\Sigma \mathbb{Z} \otimes_{\mathbb{Z}} R \text {. }
$$


Let

$$
\tau=\left(\begin{array}{ccccc}
0 & 0 & 0 & 0 & \cdots \\
1 & 0 & 0 & 0 & \cdots \\
0 & 1 & 0 & 0 & \cdots \\
0 & 0 & 1 & 0 & \cdots \\
. & . & . & . & \cdots \\
. & . & . & . & \cdots
\end{array}\right) \in \Sigma \mathbb{Z}
$$

This element is invertible since $\tau \tau^{t}=1$ in $\Sigma \mathbb{Z}$ and consequently $\tau \in$ $G L_{1}(\Sigma \mathbb{Z})$. Let $[P]$ be a generator of the group $K_{0}(R)$, where $P$ is a finitely generated projective $R$-module. There is an $R$-module $Q$ such that $P \oplus Q \cong R^{n}$ for some $n$ and an $R$-module homomorphism $R^{n} \rightarrow R^{n}$ which is the identity on $P$ and trivial on $Q$. Let us call $p$ the $n \times n$ matrix with coefficients in $R$ corresponding to that homomorphism. By using the tensor product of matrices, one can construct the element $\tau \otimes p+$ $1 \otimes(1-p)$, which is an invertible $n \times n$ matrix with coefficients in $\Sigma R$, in other words, which belongs to $G L(\Sigma R)$. This produces a homomorphism

$$
\theta: K_{0}(R) \rightarrow K_{1}(\Sigma R)
$$

which sends $[P]$ to the class of $\tau \otimes p+1 \otimes(1-p)$ in $K_{1}(\Sigma R)=$ $G L(\Sigma R) / E(\Sigma R)$.

Proposition 4.8. The homomorphism $\theta: K_{0}(R) \rightarrow K_{1}(\Sigma R)$ is an isomomorphism.

This fact is proved in [53] and can be generalized. Let $\sigma: \mathbb{Z} \rightarrow$ $G L_{1}(\Sigma \mathbb{Z})$ be the group homomorphism given by $\sigma(1)=\tau$. It induces a map $\sigma^{+}: S^{1} \simeq B \mathbb{Z}^{+} \rightarrow B G L(\Sigma \mathbb{Z})^{+}$and we write $\widetilde{\varepsilon}_{R}$ for the composition

$$
\widetilde{\varepsilon}_{R}: S^{1} \wedge B G L(R)^{+} \stackrel{\sigma^{+} \wedge \mathrm{id}}{\longrightarrow} B G L(\Sigma \mathbb{Z})^{+} \wedge B G L(R)^{+} \stackrel{\widehat{\gamma}^{\Sigma \mathbb{Z}, R}}{\longrightarrow} B G L(\Sigma R)^{+} .
$$

For any generator $[P]$ of $K_{0}(R)$, let us choose a representative $\zeta_{P}: S^{1} \rightarrow$ $B G L(\Sigma R)^{+}$of the element $\theta([P]) \in K_{1}(\Sigma R)=\pi_{1}\left(B G L(\Sigma R)^{+}\right)$. This defines a map

$$
\varepsilon_{R}^{\prime}: S^{1} \wedge\left(K_{0}(R) \times B G L(R)^{+}\right) \longrightarrow K_{0}(\Sigma R) \times B G L(\Sigma R)^{+}
$$

given by $\varepsilon_{R}^{\prime}(t \wedge([P], x))=\left(0, \zeta_{P}(t)+\widetilde{\varepsilon}_{R}(t \wedge x)\right)$. Its adjoint is

$$
\varepsilon_{R}: K_{0}(R) \times B G L(R)^{+} \longrightarrow \Omega\left(K_{0}(\Sigma R) \times B G L(\Sigma R)^{+}\right) .
$$

The point is that the homotopy type of the space $B G L(\Sigma R)^{+}$depends very strongly on the homotopy type of $B G L(R)^{+}$because of the following result. 
Theorem 4.9. The map $\varepsilon_{R}$ is a natural homotopy equivalence:

$$
\varepsilon_{R}: K_{0}(R) \times B G L(R)^{+} \stackrel{\simeq}{\longrightarrow} \Omega\left(K_{0}(\Sigma R) \times B G L(\Sigma R)^{+}\right) .
$$

Proof: See [59, Théorème 1.4.9 and Théorème 2.3.5]; see also [96, Section 3].

Theorem 4.9 immediately implies the following consequence.

Corollary 4.10. For any ring $R$ and for any integer $i \geq 0$, there is an isomorphism

$$
K_{i}(R) \cong K_{i+1}(\Sigma R)
$$

Proof: By definition,

$$
\begin{aligned}
K_{i}(R)=\pi_{i}\left(K_{0}(R) \times B G L(R)^{+}\right) & \cong \pi_{i+1}\left(K_{0}(\Sigma R) \times B G L(\Sigma R)^{+}\right) \\
& \cong \pi_{i+1}\left(B G L(\Sigma R)^{+}\right)=K_{i+1}(\Sigma R)
\end{aligned}
$$

for any integer $i \geq 0$.

If one takes the 0-connected cover of both sides of the equivalence provided by Theorem 4.9 and applies Theorem 3.13, one gets:

Corollary 4.11. There is a natural homotopy equivalence

$$
B G L(R)^{+} \simeq \Omega B E(\Sigma R)^{+} .
$$

Remark 4.12. Of course, Theorem 4.9 shows that for any $\operatorname{ring} R$, the space $K_{0}(R) \times B G L(R)^{+}$is an infinite loop space since $K_{0}(R) \times B G L(R)^{+} \simeq$ $\Omega\left(K_{0}(\Sigma R) \times B G L(\Sigma R)^{+}\right) \simeq \Omega^{2}\left(K_{0}\left(\Sigma^{2} R\right) \times B G L\left(\Sigma^{2} R\right)^{+}\right) \simeq \cdots$. This enables us to define an $\Omega$-spectrum whose 0 -th space is the space $K_{0}(R) \times$ $B G L(R)^{+}$.

Definition 4.13. For any $\operatorname{ring} R$, the $K$-theory spectrum of $R$ is the $\Omega$-spectrum $K_{R}$ whose $n$-th space is $\left(K_{R}\right)_{n}=K_{0}\left(\Sigma^{n} R\right) \times B G L\left(\Sigma^{n} R\right)^{+}$ for all $n \geq 0$.

We shall also use the 0 -connected cover $X_{R}$ of the $K$-theory spectrum $K_{R}$ of a ring $R$.

Definition 4.14. For any ring $R$, the 0 -connected $K$-theory spectrum of $R$ is the $\Omega$-spectrum $X_{R}$ whose $n$-th space is $\left(X_{R}\right)_{n}=B G L\left(\Sigma^{n} R\right)^{+}(n)$ for all $n \geq 0$. Here, $X(n)$ is written for the $n$-th connected cover of a CW-complex $X$, i.e., the homotopy fiber of the $n$-th Postnikov section $X \rightarrow X[n]$ of $X$ (see Section 7): this means that $X(n)$ is $n$-connected and that $\pi_{i}(X(n)) \cong \pi_{i}(X)$ for all $i \geq n$. 
Of course, since these spectra are $\Omega$-spectra, their homotopy groups are

$$
\begin{aligned}
\pi_{i}\left(K_{R}\right) & =\underset{n}{\lim } \pi_{i+n}\left(\left(K_{R}\right)_{n}\right) \\
& =\underset{n}{\lim } \pi_{i+n}\left(K_{0}\left(\Sigma^{n} R\right) \times B G L\left(\Sigma^{n} R\right)^{+}\right) \cong \underset{n}{\lim _{\longrightarrow}} K_{i+n}\left(\Sigma^{n} R\right)
\end{aligned}
$$

for any $i \in \mathbb{Z}$; in particular, they are in general non-trivial if $i<0$. On the other hand, for $X_{R}$,

$$
\pi_{i}\left(X_{R}\right)=\underset{n}{\lim } \pi_{i+n}\left(\left(X_{R}\right)_{n}\right)=\underset{n}{\lim } \pi_{i+n}\left(B G L\left(\Sigma^{n} R\right)^{+}(n)\right)
$$

and we may conclude that $\pi_{i}\left(X_{R}\right)=0$ if $i \leq 0$ since $B G L\left(\Sigma^{n} R\right)^{+}(n)$ is $n$-connected and that

$$
\pi_{i}\left(X_{R}\right) \cong \underset{n}{\lim } \pi_{i+n}\left(B G L\left(\Sigma^{n} R\right)^{+}\right) \cong \underset{n}{\lim } K_{i+n}\left(\Sigma^{n} R\right)
$$

if $i \geq 1$. Therefore, Corollary 4.10 implies the following result for $i \geq 0$.

\section{Theorem 4.15.}

(a) For any integer $i \geq 0, K_{i}(R) \cong \pi_{i}\left(K_{R}\right)$.

(b) For any integer $i \geq 1, K_{i}(R) \cong \pi_{i}\left(X_{R}\right)$.

Remark 4.16. There are many other constructions of the $K$-theory spectrum of a ring $R$ : see for instance [29, Chapter 11], [45], or [96, Section 3].

Remark 4.17. Since the $K$-groups of a ring $R$ in positive dimensions are the homotopy groups of its 0 -connected $K$-theory spectrum $X_{R}$ (or of $K_{R}$ ), they are very strongly related to the homology groups of $X_{R}$ via the stable Hurewicz homomorphism, as we shall see in Section 6. Therefore, it would be extremely useful to be able to compute

$$
H_{i}\left(X_{R} ; \mathbb{Z}\right)=\underset{n}{\lim } H_{i+n}\left(\left(X_{R}\right)_{n} ; \mathbb{Z}\right)=\underset{n}{\lim } H_{i+n}\left(B G L\left(\Sigma^{n} R\right)^{+}(n) ; \mathbb{Z}\right) .
$$

In a recent paper [63] which generalizes MacLane's $Q$-construction for computing the stable homology of Eilenberg-Maclane spaces [60], R. McCarthy obtains an explicit chain complex whose homology is the homology of $X_{R}$. This promising idea should provide more information on $H_{i}\left(X_{R} ; \mathbb{Z}\right)$ and consequently on the algebraic $K$-groups $K_{i}(R)$.

Let us conclude this section by explaining that the product structure in the $K$-theory of rings may also be expressed in terms of $K$-theory spectra. 
Definition 4.18. Let us consider two rings $R$ and $R^{\prime}$, together with their associated 0 -connected spectra $X_{R}$ and $X_{R^{\prime}}$, and let $S$ denote the sphere spectrum. The external product

$$
\wedge: \pi_{i}\left(X_{R}\right) \otimes \pi_{j}\left(X_{R^{\prime}}\right) \longrightarrow \pi_{i+j}\left(X_{R} \wedge X_{R^{\prime}}\right)
$$

is defined as follows. If $x \in \pi_{i}\left(X_{R}\right)$ and $y \in \pi_{j}\left(X_{R^{\prime}}\right)$ are represented by maps of spectra $\alpha: S \rightarrow X_{R}$ of degree $i$ and $\beta: S \rightarrow X_{R^{\prime}}$ of degree $j$ respectively, $x \wedge y$ is then the class in $\pi_{i+j}\left(X_{R} \wedge X_{R^{\prime}}\right)$ represented by the map $\alpha \wedge \beta: S \simeq S \wedge S \rightarrow X_{R} \wedge X_{R^{\prime}}$ of degree $i+j$ (see [94, p. 270]).

Definition 4.19. The map

$$
\widehat{\gamma}^{R, R^{\prime}}: B G L(R)^{+} \wedge B G L\left(R^{\prime}\right)^{+} \longrightarrow B G L\left(R \otimes R^{\prime}\right)^{+}
$$

which was the key ingredient in Definition 4.2 extends of course to a map $\widehat{\gamma}^{\Sigma^{n} R, \Sigma^{m} R^{\prime}}: B G L\left(\Sigma^{n} R\right)^{+} \wedge B G L\left(\Sigma^{m} R^{\prime}\right)^{+} \longrightarrow B G L\left(\Sigma^{n+m}\left(R \otimes R^{\prime}\right)\right)^{+}$ for all $n$ and $m$ and consequently to a pairing of spectra

$$
\bar{\gamma}^{R, R^{\prime}}: X_{R} \wedge X_{R^{\prime}} \longrightarrow X_{R \otimes R^{\prime}}
$$

which we call the Loday pairing (see [59, Proposition 2.4.2]).

Therefore, the definition of the $K$-theoretical product introduced in Definition 4.2 and Remark 4.4 can be formulated as follows.

Corollary 4.20. For all rings $R$ and $R^{\prime}$ and for all positive integers $i$ and $j$, the $K$-theoretical product is given by

$$
\begin{aligned}
\star: K_{i}(R) \otimes K_{j}\left(R^{\prime}\right) \cong \pi_{i}\left(X_{R}\right) \otimes \pi_{j}\left(X_{R^{\prime}}\right) \stackrel{\wedge}{\longrightarrow} \pi_{i+j}\left(X_{R} \wedge X_{R^{\prime}}\right) \\
\stackrel{\left(\bar{\gamma}^{R, R^{\prime}}\right)_{*}}{\longrightarrow} \pi_{i+j}\left(X_{R \otimes R^{\prime}}\right) \cong K_{i+j}\left(R \otimes R^{\prime}\right) .
\end{aligned}
$$

Moreover, if $R$ is commutative, the ring structure of $K_{*}(R)$ is given by

$$
\begin{aligned}
\star: K_{i}(R) \otimes K_{j}(R) & \cong \pi_{i}\left(X_{R}\right) \otimes \pi_{j}\left(X_{R}\right) \stackrel{\wedge}{\longrightarrow} \pi_{i+j}\left(X_{R} \wedge X_{R}\right) \\
& \stackrel{\left(\bar{\gamma}^{R, R}\right)_{*}}{\longrightarrow} \pi_{i+j}\left(X_{R \otimes R}\right) \cong K_{i+j}(R \otimes R) \stackrel{\nabla_{*}}{\longrightarrow} K_{i+j}(R)
\end{aligned}
$$

for all $i, j \geq 1$.

\section{The algebraic $K$-theory of finite fields}

When D. Quillen introduced the higher algebraic $K$-groups, one of his great achievements was to completely compute them for finite fields (see $[\mathbf{7 1}])$. Let $p$ be a prime, $q$ a power of $p$ and let $\mathbb{F}_{q}$ denote the field with $q$ elements. Since $\mathbb{F}_{q}$ is a field, it is known by Theorem 1.2 that

$$
K_{0}\left(\mathbb{F}_{q}\right) \cong \mathbb{Z}
$$


In order to calculate $K_{i}\left(\mathbb{F}_{q}\right)$ for any positive integer $i$, Quillen's brilliant idea was to construct a topological model for the space $B G L\left(\mathbb{F}_{q}\right)^{+}$using well known spaces. He considered the classifying space $B U$ of the infinite unitary group $U$ and the Adams operation $\Psi^{q}: B U \rightarrow B U$. Remember that for $i \geq 1 \pi_{i}(B U)=0$ if $i$ is odd and $\pi_{i}(B U) \cong \mathbb{Z}$ if $i$ is even (by Bott periodicity, see [35] or Chapter 10 of [51]), and that the homomorphism $\Psi_{*}^{q}: \pi_{2 j}(B U) \rightarrow \pi_{2 j}(B U)$ induced by $\Psi^{q}$ is multiplication by $q^{j}$ (see [1, Corollary 5.2]).

Definition 5.1. For any integer $q \geq 2$, let $F \Psi^{q}$ be the pull-back of the diagram

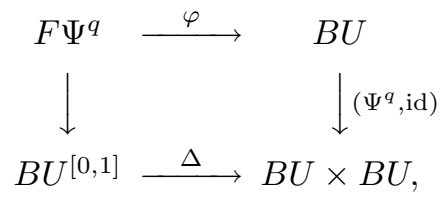

where $B U^{[0,1]}$ is the path space of $B U$ and $\Delta$ the map sending a path in $B U$ to its endpoints. A point of $F \Psi^{q}$ is a pair $(x, u)$, where $x$ is a point of $B U$ and $u$ a path in $B U$ joining $\Psi^{q}(x)$ to $x$. In other words, $F \Psi^{q}$ is the homotopy theoretical fixpoint set of $\Psi^{q}$. According to Lemma 1 of [71], it turns out that if $d: B U \times B U \rightarrow B U$ is the map defined by $d(x, y)=x-y$, then $F \Psi^{q}$ is the homotopy fiber of the composition

$$
\Psi^{q}-1: B U \stackrel{\left(\Psi^{q}, \mathrm{id}\right)}{\longrightarrow} B U \times B U \stackrel{d}{\longrightarrow} B U .
$$

Proposition 5.2. For any integer $q \geq 2$, the space $F \Psi^{q}$ is simple and its homotopy groups are

$$
\pi_{i}\left(F \Psi^{q}\right) \cong \begin{cases}0, & \text { if } i \text { is an even integer } \geq 2, \\ \mathbb{Z} /\left(q^{j}-1\right), & \text { if } i \text { is an odd integer } \\ \quad \text { of the form } i=2 j-1 \text { with } j \geq 1 .\end{cases}
$$

Proof: Let us consider the fibration

$$
F \Psi^{q} \stackrel{\varphi}{\longrightarrow} B U \stackrel{\Psi^{q}-1}{\longrightarrow} B U
$$

Since the action of $\pi_{1}\left(F \Psi^{q}\right)$ on the higher homotopy groups $\pi_{i}\left(F \Psi^{q}\right)$ comes from the action of $\pi_{1}(B U)$ on $\pi_{i}\left(F \Psi^{q}\right)$, it is trivial because $B U$ is simply connected. The calculation of $\pi_{i}\left(F \Psi^{q}\right)$ directly follows from the 
homotopy exact sequence of the above fibration

$$
\begin{aligned}
& \cdots \longrightarrow \underbrace{\pi_{2 j+1}(B U)}_{=0} \longrightarrow \pi_{2 j}\left(F \Psi^{q}\right) \longrightarrow \underbrace{\pi_{2 j}(B U)}_{\cong \mathbb{Z}} \\
& \stackrel{q^{j}-1}{\longrightarrow} \underbrace{\pi_{2 j}(B U)}_{\cong \mathbb{Z}} \longrightarrow \pi_{2 j-1}\left(F \Psi^{q}\right) \longrightarrow \underbrace{\pi_{2 j-1}(B U)}_{=0} \longrightarrow \cdots
\end{aligned}
$$

for any positive integer $j$.

From now on, let $p$ be a prime, $q$ a power of $p$ and fix a prime $l$ such that $l \neq p$. Quillen's main argument is based on the calculation of the cohomology of the space $F \Psi^{q}$ and of the infinite general linear group $G L\left(\mathbb{F}_{q}\right)$. Let us start by defining some classes in the cohomology of $F \Psi^{q}$. It is well known that

$$
H^{*}(B U ; \mathbb{Z}) \cong \mathbb{Z}\left[\widehat{c}_{1}, \widehat{c}_{2}, \widehat{c}_{3}, \ldots\right]
$$

and

$$
H^{*}(B U ; \mathbb{Z} / l) \cong \mathbb{Z} / l\left[\bar{c}_{1}, \bar{c}_{2}, \bar{c}_{3}, \ldots\right]
$$

where the $\widehat{c}_{i}$ 's and the $\bar{c}_{i}$ 's are the integral universal Chern classes, respectively the $\bmod l$ universal Chern classes, of degree $2 i$ (see [66, Chapter 14]).

Definition 5.3. For any positive integer $i$, the $i$-th integral Chern class of $F \Psi^{q}$ is

$$
\widetilde{c}_{i}=\varphi^{*}\left(\widehat{c}_{i}\right) \in H^{2 i}\left(F \Psi^{q} ; \mathbb{Z}\right)
$$

and the $i$-th $\bmod l$ Chern class of $F \Psi^{q}$ is

$$
c_{i}=\varphi^{*}\left(\bar{c}_{i}\right) \in H^{2 i}\left(F \Psi^{q} ; \mathbb{Z} / l\right),
$$

where $\varphi^{*}: H^{*}(B U ; A) \rightarrow H^{*}\left(F \Psi^{q} ; A\right)$ is the homomorphism induced by $\varphi$, for $A=\mathbb{Z}$ and $A=\mathbb{Z} / l$ respectively.

The diagram occuring in Definition 5.1 induces the following commutative diagram for any abelian group $A$ in which the columns are exact 
sequences of pairs:

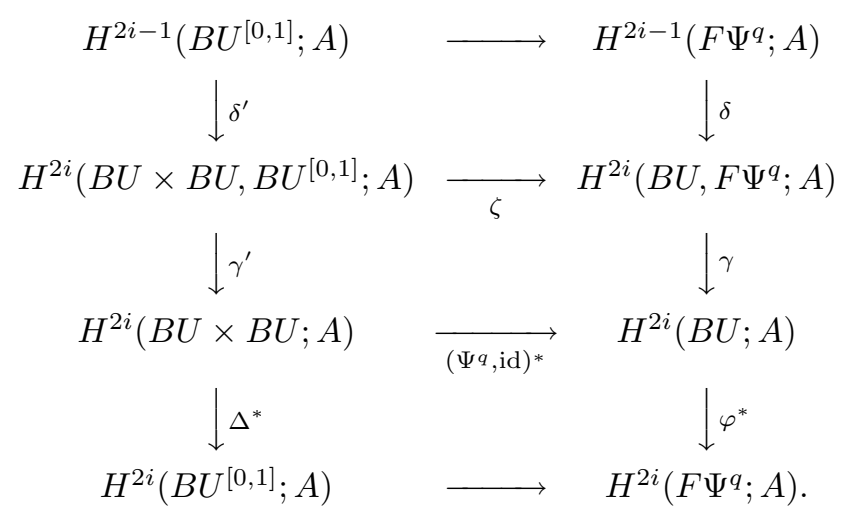

In this diagram, $\gamma^{\prime}$ and $\delta$ are injective because $H^{2 i-1}\left(B U^{[0,1]} ; A\right)=$ $H^{2 i-1}(B U ; A)=0$. Let us first take $A=\mathbb{Z}$. Since $\Delta: B U^{[0,1]} \rightarrow B U \times$ $B U$ is homotopy equivalent to the diagonal map $B U \rightarrow B U \times B U$ sending a point $x$ to $(x, x)$, the induced homomorphism $\Delta^{*}: H^{2 i}(B U \times B U ; \mathbb{Z}) \rightarrow$ $H^{2 i}\left(B U^{[0,1]} ; \mathbb{Z}\right)$ satisfies $\Delta^{*}\left(\widehat{c}_{i} \otimes 1-1 \otimes \widehat{c}_{i}\right)=0$. Consequently, there is a unique element $z \in H^{2 i}\left(B U \times B U, B U^{[0,1]} ; \mathbb{Z}\right)$ such that $\gamma^{\prime}(z)=$ $\widehat{c}_{i} \otimes 1-1 \otimes \widehat{c}_{i}$. Thus, the commutativity of the diagram shows that $\zeta(z) \in H^{2 i}\left(B U, F \Psi^{q} ; \mathbb{Z}\right)$ has the property that $\gamma \zeta(z)=\left(q^{i}-1\right) \widehat{c}_{i} \in$ $H^{2 i}(B U ; \mathbb{Z})$. Now, consider both coefficients $A=\mathbb{Z}$ and $A=\mathbb{Z} /\left(q^{i}-1\right)$, and the diagram

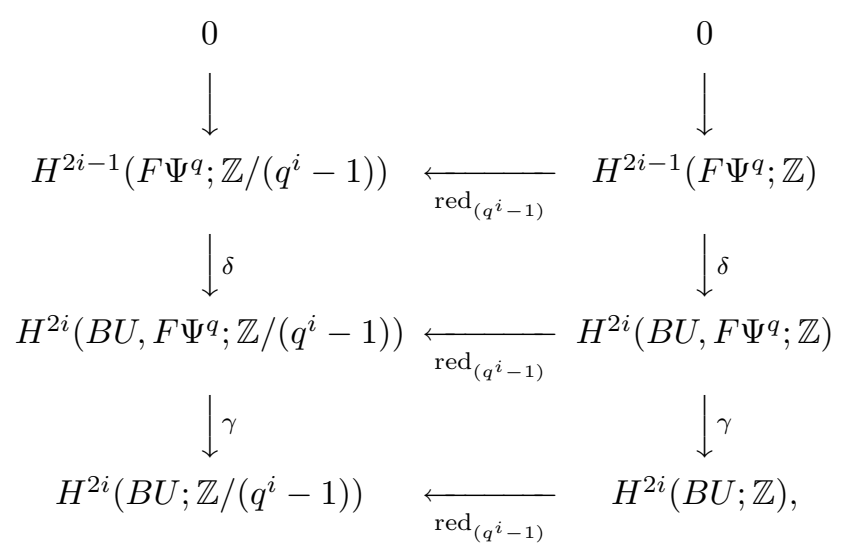

where the horizontal homomorphisms are induced by the reduction $\bmod \left(q^{i}-1\right)$. It follows from the commutativity of the diagram that $\gamma \operatorname{red}_{\left(q^{i}-1\right)} \zeta(z)=\operatorname{red}_{\left(q^{i}-1\right)}\left(\left(q^{i}-1\right) \widehat{c}_{i}\right)=0$. 
Definition 5.4. For any positive integer $i$, there is a unique element

$$
\widetilde{e}_{i} \in H^{2 i-1}\left(F \Psi^{q} ; \mathbb{Z} /\left(q^{i}-1\right)\right)
$$

such that $\delta\left(\widetilde{e}_{i}\right)=\operatorname{red}_{\left(q^{i}-1\right)} \zeta(z)$. This element is related to the integral Chern class $\widetilde{c}_{i} \in H^{2 i}\left(F \Psi^{q} ; \mathbb{Z}\right)$ by the formula

$$
\beta_{\left(q^{i}-1\right)}\left(\widetilde{e}_{i}\right)=\widetilde{c}_{i},
$$

where $\beta_{\left(q^{i}-1\right)}$ is the Bockstein homomorphism $H^{2 i-1}\left(F \Psi^{q} ; \mathbb{Z} /\left(q^{i}-1\right)\right) \rightarrow$ $H^{2 i}\left(F \Psi^{q} ; \mathbb{Z}\right)$ (see Lemmas 3 and 5 in $[\mathbf{7 1}]$ ).

Definition 5.5. Let $r$ be the smallest positive integer such that $q^{r} \equiv$ $1 \bmod l$. Then, we define for any integer $j \geq 1$

$$
e_{j r} \in H^{2 j r-1}\left(F \Psi^{q} ; \mathbb{Z} / l\right)
$$

as the image of $\widetilde{e}_{j r}$ under the homomorphism

$$
H^{2 j r-1}\left(F \Psi^{q} ; \mathbb{Z} /\left(q^{j r}-1\right)\right) \rightarrow H^{2 j r-1}\left(F \Psi^{q} ; \mathbb{Z} / l\right)
$$

induced by the obvious surjection $\mathbb{Z} /\left(q^{j r}-1\right) \rightarrow \mathbb{Z} / l$.

By using the Eilenberg-Moore spectral sequence of the fibration

$$
F \Psi^{q} \stackrel{\varphi}{\longrightarrow} B U \stackrel{\Psi^{q}-1}{\longrightarrow} B U,
$$

D. Quillen was able to calculate the cohomology of $F \Psi^{q}$ with coefficients in $\mathbb{Z} / l$.

\section{Theorem 5.6.}

(a) The monomials $c_{r}^{\alpha_{1}} c_{2 r}^{\alpha_{2}} c_{3 r}^{\alpha_{3}} \cdots e_{r}^{\beta_{1}} e_{2 r}^{\beta_{2}} e_{3 r}^{\beta_{3}} \cdots$, with $\alpha_{j} \geq 0$ and $\beta_{j}=0$ or 1 , form an additive basis for $H^{*}\left(F \Psi^{q} ; \mathbb{Z} / l\right)$.

(b) If $l$ is an odd prime or if $l=2$ and $q \equiv 1 \bmod 4$, then $e_{j r}^{2}=0$ for all $j \geq 1$ and there is an algebra isomorphism

$$
H^{*}\left(F \Psi^{q} ; \mathbb{Z} / l\right) \cong \mathbb{Z} / l\left[c_{r}, c_{2 r}, c_{3 r}, \ldots\right] \otimes \Lambda_{\mathbb{Z} / l}\left(e_{r}, e_{2 r}, e_{3 r}, \ldots\right) .
$$

(c) If $l=2$ and $q \equiv 3 \bmod 4$, one has $r=1$ and the relations

$$
e_{j}^{2}=c_{2 j-1}+\sum_{k=1}^{j-1} c_{k} c_{2 j-k-1}
$$

and there is an algebra isomorphism

$$
H^{*}\left(F \Psi^{q} ; \mathbb{Z} / 2\right) \cong \mathbb{Z} / 2\left[c_{2}, c_{4}, c_{6}, \ldots, e_{1}, e_{2}, e_{3}, \ldots\right] .
$$

Proof: See [71, Theorem 1] and [44, Section IV.8]. 
The next ingredient in Quillen's argument is the notion of the Brauer lift (see [71, Section 7]). Let $G$ be a finite group and $\rho: G \rightarrow G L_{n}\left(\mathbb{F}_{q}\right)$ a representation of $G$ over the field $\mathbb{F}_{q}$ with $q$ elements. Let us denote by $\bar{\rho}$ the representation $\bar{\rho}=\rho \otimes_{\mathbb{F}_{q}} \overline{\mathbb{F}}_{q}$ of $G$ over the algebraic closure $\overline{\mathbb{F}}_{q}$ of $\mathbb{F}_{q}$. We can look at the complex valued function on $G$ defined by $\chi_{\bar{\rho}}(g)=$ $\sum \iota\left(\lambda_{k}(g)\right)$ for $g \in G$, where $\iota$ is an embedding $\overline{\mathbb{F}}_{q}^{*} \hookrightarrow \mathbb{C}^{*}$ and $\left\{\lambda_{k}(g)\right\}$ is the set of eigenvalues of $\bar{\rho}(g)$. It turns out that $\chi_{\bar{\rho}}$ is the character of a unique virtual complex representation $\widetilde{\rho}$ of $G$; therefore, $\chi_{\bar{\rho}}$ belongs to the complex representation $\operatorname{ring} R(G)=R_{\mathbb{C}}(G)$ of $G$ and we get a homomorphism $R_{\mathbb{F}_{q}}(G) \rightarrow R(G)$ which maps the class of the character of $\rho$ to $\chi_{\bar{\rho}}$. In fact, $\widetilde{\rho}$ is stable under the Adams operation $\Psi^{q}$ (see $\left[\mathbf{7 1}\right.$, Section 7]) and the previous homomorphism is actually $R_{\mathbb{F}_{q}}(G) \rightarrow$ $R(G)^{\Psi^{q}}$. If we compose it with the classifying map $R(G) \rightarrow[B G, B U]$ sending a complex representation to the corresponding homotopy class of maps between classifying spaces, we obtain the homomorphism

$$
\tau: R_{\mathbb{F}_{q}}(G) \rightarrow[B G, B U]^{\Psi^{q}} .
$$

On the other hand, observe again the fibration $F \Psi^{q} \stackrel{\varphi}{\longrightarrow} B U \stackrel{\Psi^{q}-1}{\longrightarrow} B U$ and remember that a point of $F \Psi^{q}$ is a pair $(x, u)$, where $x$ is a point of $B U$ and $u$ a path joining $\Psi^{q}(x)$ to $x$ : this implies that for any $Y$, a map $Y \rightarrow F \Psi^{q}$ can be identified with a pair consisting of a map $f: Y \rightarrow B U$ together with a homotopy joining $\Psi^{q} f$ to $f$. Consequently, $\varphi$ induces a homomorphism

$$
\varphi_{*}:\left[Y, F \Psi^{q}\right] \longrightarrow[Y, B U]^{\Psi^{q}}
$$

which is clearly surjective. By looking at the fibration

$$
\Omega B U \simeq U \longrightarrow F \Psi^{q} \stackrel{\varphi}{\longrightarrow} B U
$$

obtained by looping the base space of the above fibration, one gets that $\varphi_{*}$ is an isomorphism if $[Y, U]=0$. Therefore, if $[B G, U]=0$, the above homomorphism $\tau$ can be viewed as a homomorphism

$$
\tau: R_{\mathbb{F}_{q}}(G) \rightarrow\left[B G, F \Psi^{q}\right] .
$$

This is the case for $G=G L_{n}\left(\mathbb{F}_{q}\right)$ and for the direct limit $G=G L\left(\mathbb{F}_{q}\right)=$ $\lim _{n} G L_{n}\left(\mathbb{F}^{q}\right)$ according to Lemma 14 of $[\mathbf{7 1}]$.

Definition 5.7. Let $G=G L_{n}\left(\mathbb{F}_{q}\right)$ and $\rho=\mathrm{id}: G L_{n}\left(\mathbb{F}_{q}\right) \rightarrow G L_{n}\left(\mathbb{F}_{q}\right)$. The Brauer lift is the homotopy class of maps $b_{n}=\tau(\mathrm{id}) \in\left[B G L_{n}\left(\mathbb{F}_{q}\right), F \Psi^{q}\right]$. By passing to the direct limit $G L\left(\mathbb{F}_{q}\right)=\lim _{n} G L_{n}\left(\mathbb{F}_{q}\right)$, one obtains a homotopy class of maps

$$
b=\tau(\mathrm{id}) \in\left[B G L\left(\mathbb{F}_{q}\right), F \Psi^{q}\right] .
$$


For simplicity, we shall also denote by $b \in\left[B G L\left(\mathbb{F}_{q}\right), B U\right]$ the composition of this last homotopy class of maps with the inclusion $\varphi: F \Psi^{q} \rightarrow B U$ and call it the Brauer lift.

This enables Quillen to prove his main result.

Theorem 5.8 (Quillen). For any prime power $q$, there is a homotopy equivalence

$$
B G L\left(\mathbb{F}_{q}\right)^{+} \simeq F \Psi^{q} .
$$

Proof: (See [71, Theorems 2, 3, 4, 5, 6 and 7] for the details.) Let $p$ be a prime, $q$ a power of $p$ and $r$ be as in Definition 5.5. The argument is based on the investigation of the map

$$
b^{+}: B G L\left(\mathbb{F}_{q}\right)^{+} \longrightarrow F \Psi^{q}
$$

induced by the the Brauer lift $b: B G L\left(\mathbb{F}_{q}\right) \longrightarrow F \Psi^{q}$ (notice that $\left(F \Psi^{q}\right)^{+} \simeq$ $F \Psi^{q}$ since $\pi_{1}\left(F \Psi^{q}\right)$ is abelian by Proposition 5.2 and contains therefore no non-trivial perfect normal subgroup). Because of Theorem 5.6, one can also compute the $\bmod l$ homology of $F \Psi^{q}$ for any prime $l \neq p$. On the other hand, using techniques from homology theory of finite groups, it is possible to calculate $H_{*}\left(G L_{n}\left(\mathbb{F}_{q}\right) ; \mathbb{Z} / l\right)$ for all positive integers $n$ and consequently $H_{*}\left(G L\left(\mathbb{F}_{q}\right) ; \mathbb{Z} / l\right) \cong \varliminf_{n} H_{*}\left(G L_{n}\left(\mathbb{F}_{q}\right) ; \mathbb{Z} / l\right)$, and to prove that the homomorphism $\left(b^{+}\right)_{*}: H_{*}\left(B G L\left(\mathbb{F}_{q}\right)^{+} ; \mathbb{Z} / l\right) \cong H_{*}\left(G L\left(\mathbb{F}_{q}\right) ; \mathbb{Z} / l\right) \longrightarrow$ $H_{*}\left(F \Psi^{q} ; \mathbb{Z} / l\right)$ induced by $b$ is an isomorphism. The next thing to do is to prove the vanishing of $H_{i}\left(G L\left(\mathbb{F}_{q}\right) ; \mathbb{Z} / p\right) \cong H_{i}\left(B G L\left(\mathbb{F}_{q}\right)^{+} ; \mathbb{Z} / p\right)$ for all $i \geq 1$. Then, apply the generalized Whitehead theorem (see [48, Corollary 1.5], or $\left[\mathbf{2 9}\right.$, Proposition 4.15]) to the map $b^{+}: B G L\left(\mathbb{F}_{q}\right)^{+} \longrightarrow F \Psi^{q}$ : since both spaces are simple according to Propositions 4.1 and 5.2, we can conclude that $b^{+}$is a homotopy equivalence if we can show that $b$ induces an isomorphism

$$
\left(b^{+}\right)_{*}: H_{*}\left(B G L\left(\mathbb{F}_{q}\right)^{+} ; \mathbb{Z}\right) \stackrel{\cong}{\longrightarrow} H_{*}\left(F \Psi^{q} ; \mathbb{Z}\right) .
$$

This holds if $b^{+}$induces an isomorphism on homology with coefficients in $\mathbb{Q}$, in $\mathbb{Z} / p$ and in $\mathbb{Z} / l$ for all primes $l \neq p$. This is already done for coefficients in $\mathbb{Z} / l$. It is easy to check that $H_{i}\left(B G L\left(\mathbb{F}_{q}\right)^{+} ; \mathbb{Q}\right) \cong H_{i}\left(G L\left(\mathbb{F}_{q}\right) ; \mathbb{Q}\right)=$ 0 for all $i \geq 1$ because $H_{i}\left(G L\left(\mathbb{F}_{q}\right) ; \mathbb{Q}\right) \cong \varliminf_{n} H_{i}\left(G L_{n}\left(\mathbb{F}_{q}\right) ; \mathbb{Q}\right)=0$ since $G L_{n}\left(\mathbb{F}_{q}\right)$ is a finite group. On the other hand, we know from Proposition 5.2 that the homotopy groups of $F \Psi^{q}$ are torsion groups which are $p$-torsion free. Thus, by Serre class theory (see [82, Chapitre I]), all integral homology groups of $F \Psi^{q}$ are also torsion groups which are $p$-torsion free: in other words, $H_{i}\left(F \Psi^{q} ; \mathbb{Q}\right)=0$ and $H_{i}\left(F \Psi^{q} ; \mathbb{Z} / p\right)=0$ for all $i \geq 1$. Thus, $b^{+}: B G L\left(\mathbb{F}_{q}\right)^{+} \rightarrow F \Psi^{q}$ is a homotopy equivalence and we get the statement of the theorem. 
This result is important because it provides a convenient topological model $F \Psi^{q}$ for the algebraic $K$-theory space $B G L\left(\mathbb{F}_{q}\right)^{+}$. In particular, an immediate consequence of it is the calculation of the algebraic $K$-groups of all finite fields: Proposition 5.2 and Theorem 5.8 imply the following result (see [71, Theorem 8]).

Corollary 5.9. For any prime power $q$, the algebraic $K$-theory of the finite field $\mathbb{F}_{q}$ is given by

$$
K_{2 i}\left(\mathbb{F}_{q}\right)=0 \quad \text { and } \quad K_{2 i-1}\left(\mathbb{F}_{q}\right) \cong \mathbb{Z} /\left(q^{i}-1\right)
$$

for all integers $i \geq 1$.

This result was the first determination of $K$-groups and initiated in some sense the research in the algebraic $K$-theory of rings.

\section{The Hurewicz homomorphism in algebraic $K$-theory}

The computation of the algebraic $K$-groups of finite fields was the first impressive $K$-theoretical result. It turns out that it is actually difficult to perform many other computations. However, this is not a surprise because the algebraic $K$-groups are homotopy groups and it is never easy to compute homotopy groups! On the other hand, there are many sophisticated techniques for the computation of the homology of groups. Notice for instance that Quillen's result on the $K$-groups of finite fields is actually based on homological calculations. Therefore, it is useful to investigate the relationships between the algebraic $K$-theory of a ring $R$ and the homology of its infinite linear groups $G L(R), E(R)$, or of its infinite Steinberg group $S t(R)$. They are exhibited by the Hurewicz homomorphisms

$$
\begin{aligned}
h_{i}: K_{i}(R)= & \pi_{i}\left(B G L(R)^{+}\right) \\
& \longrightarrow H_{i}\left(B G L(R)^{+} ; \mathbb{Z}\right) \cong H_{i}(G L(R) ; \mathbb{Z}), \quad \text { for } i \geq 1, \\
h_{i}: K_{i}(R) \cong & \pi_{i}\left(B E(R)^{+}\right) \\
& \longrightarrow H_{i}\left(B E(R)^{+} ; \mathbb{Z}\right) \cong H_{i}(E(R) ; \mathbb{Z}), \quad \text { for } i \geq 2, \\
h_{i}: K_{i}(R) \cong & \pi_{i}\left(B S t(R)^{+}\right) \\
& \longrightarrow H_{i}\left(B S t(R)^{+} ; \mathbb{Z}\right) \cong H_{i}(S t(R) ; \mathbb{Z}), \quad \text { for } i \geq 3 .
\end{aligned}
$$

Of course, since $B G L(R)^{+}, B E(R)^{+}$and $B S t(R)^{+}$are connected, simply connected and 2-connected respectively (see Theorems 3.13 and 3.14), the classical Hurewicz theorem (see [100, Theorem IV.7.1]) implies the following result. 
Theorem 6.1. For any ring $R$,

(a) $K_{1}(R) \cong H_{1}(G L(R) ; \mathbb{Z})$,

(b) $K_{2}(R) \cong H_{2}(E(R) ; \mathbb{Z})$ and $h_{3}: K_{3}(R) \rightarrow H_{3}(E(R) ; \mathbb{Z})$ is surjective,

(c) $K_{3}(R) \cong H_{3}(\operatorname{St}(R) ; \mathbb{Z})$ and $h_{4}: K_{4}(R) \rightarrow H_{4}(\operatorname{St}(R) ; \mathbb{Z})$ is surjective.

The general objective of this section is to approximate the size of the kernel and of the cokernel of $h_{i}$ in higher dimensions. We will proceed from different points of view (see also [7], [9], [12] and [14]).

Let us start by using stable homotopy theory (see also [14, Sections 1 and 2]). For any spectrum $X$, the stable Hurewicz homomorphism is a homomorphism

$$
\bar{h}_{i}: \pi_{i}(X) \longrightarrow H_{i}(X ; \mathbb{Z}),
$$

defined for all integers $i$, which fits into the long stable Whitehead exact sequence. This sequence can be defined as follows. Consider the sphere spectrum $S$. It is $(-1)$-connected with $\pi_{0}(S) \cong \mathbb{Z}$ and if we kill all its homotopy groups in positive dimensions, we get a map of spectra $\alpha_{0}: S \rightarrow H(\mathbb{Z})$ inducing an isomorphism on $\pi_{0}$, where $H(\mathbb{Z})$ is the Eilenberg-Maclane spectrum having all homotopy groups trivial except $\pi_{0}(H(\mathbb{Z})) \cong \mathbb{Z}$. The map $\alpha_{0}$ is actually the 0 -th Postnikov section of $S$ (see Section 7). The stable Hurewicz homomorphism is the homomorphism

$$
\bar{h}_{i}: \pi_{i}(X) \cong \pi_{i}(X \wedge S) \longrightarrow \pi_{i}(X \wedge H(\mathbb{Z})) \cong H_{i}(X ; \mathbb{Z})
$$

induced by the map of spectra id $\wedge \alpha_{0}: X \wedge S \rightarrow X \wedge H(\mathbb{Z})$, where id is the identity : $X \rightarrow X$. Let us write $S(0)$ for the homotopy fiber of $\alpha_{0}$ : in other words, $S(0)$ is the 0-connected cover of $S$. By taking the smash product of $X$ with the cofibration $S(0) \stackrel{\gamma_{0}}{\longrightarrow} S \stackrel{\alpha_{0}}{\longrightarrow} S[0]=H(\mathbb{Z})$, we obtain the cofibration of spectra

$$
X \wedge S(0) \stackrel{\text { id } \wedge \gamma_{0}}{\longrightarrow} X \wedge S \simeq X \stackrel{\text { id } \wedge \alpha_{0}}{\longrightarrow} X \wedge H(\mathbb{Z}) .
$$

Definition 6.2. The long stable Whitehead exact sequence of a spectrum $X$ is the homotopy exact sequence of the above cofibration:

$$
\begin{aligned}
\cdots \longrightarrow \pi_{i}(X \wedge S(0)) \stackrel{\bar{\chi}_{i}}{\longrightarrow} \pi_{i}(X) \stackrel{\bar{h}_{i}}{\longrightarrow} H_{i}(X ; \mathbb{Z}) & \\
& \stackrel{\bar{\nu}_{i}}{\longrightarrow} \pi_{i-1}(X \wedge S(0)) \longrightarrow \cdots .
\end{aligned}
$$

Here $i$ is any integer, $\bar{\chi}_{i}$ is induced by $\left(\mathrm{id} \wedge \gamma_{0}\right), \bar{h}_{i}$ is the stable Hurewicz homomorphism and $\bar{\nu}_{i}$ is the connecting homomorphism. The groups 
$\pi_{i}(X \wedge S(0))$ are usually denoted by $\Gamma_{i}(X)$ : that definition coincides actually with the homotopy groups of the homotopy fiber of the DoldThom map (see [39]) and it was recently proved in [81, Corollary 3.9], that they are isomorphic to the groups introduced in the original paper [102] by J. H. C. Whitehead.

Now, let us assume that the spectrum $X$ is $(b-1)$-connected for some integer $b$. The advantage of this approach is that one can compute the groups $\Gamma_{i}(X)$ by using the Atiyah-Hirzebruch spectral sequence for $S(0)$-homology (see [2, Section III.7]):

$$
E_{s, t}^{2} \cong H_{s}\left(X ; \pi_{t}(S(0))\right) \Longrightarrow \Gamma_{s+t}(X) .
$$

Notice that $E_{s, t}^{2}=0$ if $s \leq b-1$ or if $t \leq 0$. This reproves the Hurewicz theorem because

$$
\Gamma_{i}(X)=0 \text { for } i \leq b
$$

and consequently $\bar{h}_{i}$ is an isomorphism for $i \leq b$ and an epimorphism for $i=b+1$.

Remark 6.3. For $i=b+1$, we get

$$
\Gamma_{b+1}(X) \cong E_{b, 1}^{2} \cong \pi_{b}(X) \otimes \pi_{1}(S) \cong \pi_{b}(X) \otimes \mathbb{Z} / 2
$$

for any $(b-1)$-connected spectrum $X$ (this was already known by J. H. C. Whitehead for any $(b-1)$-connected spectrum or for any $(b-1)$-connected space with $b \geq 3$, see [102, p. 81], or [101]). Thus, the homomorphism $\bar{\chi}_{b+1}$ is actually a homomorphism from $\pi_{b}(X) \otimes \pi_{1}(S)$ to $\pi_{b+1}(X)$. Consider the commutative diagram

$$
\begin{array}{rlc}
H_{b}(X ; \mathbb{Z}) \otimes H_{1}(S(0) ; \mathbb{Z}) & \stackrel{\wedge}{\cong} & \left.H_{b+1}(X \wedge S(0) ; \mathbb{Z})\right) \\
\uparrow \cong & & \uparrow \cong \\
\pi_{b}(X) \otimes \pi_{1}(S(0)) & \stackrel{\wedge}{\longrightarrow} \pi_{b+1}(X \wedge S(0))=\Gamma_{b+1}(X) \\
\cong \downarrow(\mathrm{id})_{*} \otimes\left(\gamma_{0}\right)_{*} & & \downarrow \bar{\chi}_{b+1} \\
\pi_{b}(X) \otimes \pi_{1}(S) & \stackrel{\wedge}{\longrightarrow} & \pi_{b+1}(X \wedge S) \cong \pi_{b+1}(X),
\end{array}
$$

in which $\wedge$ is the external product (see Definition 4.18 or [94, p. 270]). The top horizontal homomorphism is an isomorphism by Künneth formula and the two top vertical arrows, which are Hurewicz homomorphisms, are isomorphisms since $X$ is $(b-1)$-connected, $S(0)$ is 0 -connected and $X \wedge S(0)$ is $b$-connected. Consequently, the external product in the middle of the diagram is an isomorphism. The homomorphism $(\mathrm{id})_{*} \otimes\left(\gamma_{0}\right)_{*}$ is an isomorphism because $\left(\gamma_{0}\right)_{*}: \pi_{1}(S(0)) \stackrel{\cong}{\longrightarrow} \pi_{1}(S)$. 
Therefore, $\bar{\chi}_{b+1}$ may be identified with the external product $\pi_{b}(X) \otimes \pi_{1}(S) \stackrel{\wedge}{\longrightarrow} \pi_{b+1}(X)$. Thus, we proved the following result.

Proposition 6.4. For any $(b-1)$-connected spectrum $X$, the sequence

$$
\begin{aligned}
\cdots \longrightarrow \Gamma_{b+2}(X) \stackrel{\bar{\chi}_{b+2}}{\longrightarrow} \pi_{b+2}(X) \stackrel{\bar{h}_{b+2}}{\longrightarrow} H_{b+2}(X ; \mathbb{Z}) \stackrel{\bar{\nu}_{b+2}}{\longrightarrow} \pi_{b}(X) \otimes \pi_{1}(S) \\
\stackrel{\wedge}{\longrightarrow} \pi_{b+1}(X) \stackrel{\bar{h}_{b+1}}{\longrightarrow} H_{b+1}(X ; \mathbb{Z}) \longrightarrow 0
\end{aligned}
$$

is exact. Observe in particular that $2\left(\operatorname{ker} \bar{h}_{b+1}\right)=0$ and $2\left(\operatorname{coker} \bar{h}_{b+2}\right)=$ 0 .

Our first goal is to show that the spectral sequence

$$
E_{s, t}^{2} \cong H_{s}\left(X ; \pi_{t}(S(0))\right) \Longrightarrow \Gamma_{s+t}(X)
$$

provides a generalization of that result for the exponent of all Gamma groups of $X$.

Definition 6.5. For any positive integer $j$, let $e_{j}$ be the exponent of the $j$-th homotopy group $\pi_{j}(S)$ of the sphere spectrum $S$. For any positive integer $i$, let $\bar{e}_{i}$ denote the product $\bar{e}_{i}=e_{1} e_{2} e_{3} \cdots e_{i}$. Notice that a prime $p$ divides $\bar{e}_{i}$ if and only if $p \leq \frac{i+3}{2}$ according to Serre's theorem on the stable homotopy groups of spheres (see [82, Section IV.6, Proposition 11]).

Now, if you look at the $E^{2}$-term $E_{s, t}^{2} \cong H_{s}\left(X ; \pi_{t} S(0)\right)$ of the AtiyahHirzebruch spectral sequence for a $(b-1)$-connected spectrum $X$, it is obvious that the product of the exponents of the groups $E_{s, t}^{2}$, for $s+t=i$ with $t \geq 1$ and $s \geq b$, kills the Gamma group $\Gamma_{i}(X)$. Because

$$
e_{t} E_{s, t}^{2}=0 \quad \text { for any } \quad t \geq 1
$$

by Definition 6.5 , since $\pi_{t}(S(0)) \cong \pi_{t}(S)$ when $t \geq 1$, we conclude that the exponent of $\Gamma_{i}(X)$ divides the product $e_{1} e_{2} e_{3} \cdots e_{i-b}$. This immediately implies the following result which was also proved by a different argument in [81, Theorem 4.3], and in [12, Theorem 4.1].

Theorem 6.6. Let $X$ be $a(b-1)$-connected spectrum. Then

$$
\bar{e}_{i-b} \Gamma_{i}(X)=0
$$

for all integers $i \geq b+1$ and the stable Hurewicz homomorphism $\bar{h}_{i}$ : $\pi_{i}(X) \rightarrow H_{i}(X ; \mathbb{Z})$ satisfies:

(a) $\bar{e}_{i-b}\left(\operatorname{ker} \bar{h}_{i}\right)=0$ for all integers $i \geq b+1$,

(b) $\bar{e}_{i-b-1}\left(\right.$ coker $\left.\bar{h}_{i}\right)=0$ for all integers $i \geq b+2$. 
Of course, we want to apply this theorem to the $K$-theory spectrum. Let us consider again the 0 -connected $K$-theory spectrum $X_{R}$ of any ring $R$ (see Definition 4.14) and let us kill its first homotopy group: we get the 1 -connected $K$-theory spectrum $X_{R}(1)$. The above argument enables us to study the stable Hurewicz homomorphism $\bar{h}_{i}: K_{i}(R) \cong$ $\pi_{i}\left(X_{R}(1)\right) \rightarrow H_{i}\left(X_{R}(1) ; \mathbb{Z}\right)$ which is an isomorphism if $i=2$. Theorem 6.6 holds here with $b=2$.

Corollary 6.7. For any ring $R$, the stable Hurewicz homomorphism $\bar{h}_{i}$ : $K_{i}(R) \rightarrow H_{i}\left(X_{R}(1) ; \mathbb{Z}\right)$ satisfies:

(a) $\bar{e}_{i-2}\left(\operatorname{ker} \bar{h}_{i}\right)=0$ for all integers $i \geq 3$,

(b) $\bar{e}_{i-3}\left(\right.$ coker $\left.\bar{h}_{i}\right)=0$ for all integers $i \geq 4$.

In particular, the exponent of the kernel, respectively of the cokernel, of $\bar{h}_{i}$ is only divisible by primes $p \leq \frac{i+1}{2}$, respectively by primes $p \leq \frac{i}{2}$. This can be formulated in another way.

Definition 6.8. For any ring $R$, for any abelian group $A$ and for any positive integer $i$, the $i$-th algebraic $K$-group of $R$ with coefficients in $A$ is the $i$-th homotopy group of $B G L(R)^{+}$or $X_{R}$ with coefficients in $A$ (see $[\mathbf{3 6}]$ and $[\mathbf{6 9}])$ :

$$
K_{i}(R ; A)=\pi_{i}\left(B G L(R)^{+} ; A\right) \cong \pi_{i}\left(X_{R} ; A\right) .
$$

In particular, if $\mathbb{Z}_{(p)}$ denotes the ring of integers localized at $p$, then $K_{i}\left(R ; \mathbb{Z}_{(p)}\right) \cong K_{i}(R) \otimes \mathbb{Z}_{(p)}$ (see [36, Theorem 1.8], or [69, Proposition 1.4]) and Corollary 6.7 shows (see also [12, Corollary 5.1]):

Corollary 6.9. For any ring $R$ and any integer $i \geq 2$,

$$
K_{i}\left(R ; \mathbb{Z}_{(p)}\right) \cong H_{i}\left(X_{R}(1) ; \mathbb{Z}_{(p)}\right)
$$

for all prime numbers $p \geq \frac{i}{2}+1$.

On the other hand, we can also deduce from the above considerations some information on the unstable Hurewicz homomorphism

$$
h_{i}: K_{i}(R) \cong \pi_{i}\left(B E(R)^{+}\right) \longrightarrow H_{i}\left(B E(R)^{+} ; \mathbb{Z}\right) \cong H_{i}(E(R) ; \mathbb{Z})
$$

for $i \geq 2$. Since $B E(R)^{+}$is the 0 -th space of the $\Omega$-spectrum $X_{R}(1)$, we can look at the following commutative diagram for all integers $i \geq 2$ :

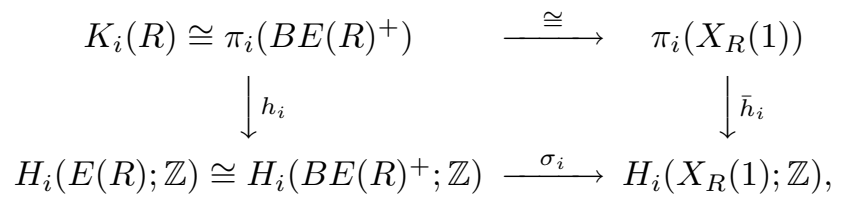


where $\sigma_{i}$ is the iterated homology suspension (see [100, Section VII.6 and Chapter VIII]). In order to state the next result, define $\widetilde{h}_{i}: K_{i}(R) \rightarrow$ $H_{i}(E(R) ; \mathbb{Z}) /\left(\operatorname{ker} \sigma_{i}\right)$ as the composition of $h_{i}: K_{i}(R) \rightarrow H_{i}\left(B E(R)^{+} ; \mathbb{Z}\right) \cong$ $H_{i}(E(R) ; \mathbb{Z})$ with the quotient map $H_{i}(E(R) ; \mathbb{Z}) \rightarrow H_{i}(E(R) ; \mathbb{Z}) /\left(\operatorname{ker} \sigma_{i}\right)$.

Corollary 6.10. For any ring $R$, the unstable Hurewicz homomorphism $h_{i}: K_{i}(R) \rightarrow H_{i}(E(R) ; \mathbb{Z})$ satisfies:

(a) $\bar{e}_{i-2}\left(\operatorname{ker} h_{i}\right)=0$ for all integers $i \geq 3$,

(b) $\bar{e}_{i-3}\left(\right.$ coker $\left.\widetilde{h}_{i}\right)=0$ for all integers $i \geq 4$,

(c) for all integers $i \geq 4$ and for any integral homology class $x \in$ $H_{i}(E(R) ; \mathbb{Z})$, there exists an element $y$ in the image of $h_{i}: K_{i}(R) \rightarrow$ $H_{i}(E(R) ; \mathbb{Z})$ and an element $z$ in the kernel of the iterated homology suspension $\sigma_{i}: H_{i}(E(R) ; \mathbb{Z}) \rightarrow H_{i}\left(X_{R}(1) ; \mathbb{Z}\right)$ such that $\bar{e}_{i-3} x=$ $y+z$.

Proof: (See also [12, Corollary 5.2].) Because of the commutativity of the above diagram, Corollary 6.7 (a) implies assertion (a) since $\operatorname{ker} h_{i}$ is contained in $\operatorname{ker} \bar{h}_{i}$. Assertions (b) and (c) follow from Corollary $6.7(\mathrm{~b})$.

If one works with coefficients in $\mathbb{Z}_{(p)}$, where $p$ is a prime $\geq \frac{i}{2}+1$, the composition $\sigma_{i} h_{i}$ is an isomorphism according to Corollary 6.9 and one gets immediately:

Corollary 6.11. For any ring $R$ and any integer $i \geq 2$, the unstable Hurewicz homomorphism $h_{i}: K_{i}\left(R ; \mathbb{Z}_{(p)}\right) \rightarrow H_{i}\left(E(R) ; \mathbb{Z}_{(p)}\right)$ is a split injection for all prime numbers $p \geq \frac{i}{2}+1$.

Our second approach of the understanding of the Hurewicz homomorphism is based on the study of the relationships between its kernel and products in algebraic $K$-theory of the form

$$
\star: K_{i}(R) \otimes K_{j}(\mathbb{Z}) \longrightarrow K_{i+j}(R \otimes \mathbb{Z}) \cong K_{i+j}(R)
$$

which have been defined in Definition 4.2 and Corollary 4.20.

Theorem 6.12. For any ring $R$ and any integer $i \geq 2$, the image of the product homomorphism

$$
\star: K_{i}(R) \otimes K_{1}(\mathbb{Z}) \longrightarrow K_{i+1}(R)
$$

is contained in the kernel of the unstable Hurewicz homomorphism

$$
h_{i+1}: K_{i+1}(R) \longrightarrow H_{i+1}(G L(R) ; \mathbb{Z}) \text {. }
$$


Proof: Let us denote by $K_{\mathbb{Z}}(-1)$ the $(-1)$-connected $K$-theory spectrum of $\mathbb{Z}$, the 0 -th space of which is $B G L(\mathbb{Z})^{+} \times K_{0}(\mathbb{Z})$ : it is a ring spectrum with unit $\eta: S \rightarrow K_{\mathbb{Z}}(-1)$ whose 0 -connected cover $S(0) \rightarrow$ $X_{\mathbb{Z}}$ is the map of spectra induced by the map of infinite loop spaces $\left(B \Sigma_{\infty}\right)^{+} \rightarrow B G L(\mathbb{Z})^{+}$which comes from the obvious inclusion of the infinite symmetric group $\Sigma_{\infty}$ into $G L(\mathbb{Z})$. This map $\eta$ induces an isomorphism $\eta_{*}: \pi_{1}(S) \stackrel{\cong}{\longrightarrow} \pi_{1}\left(K_{\mathbb{Z}}(-1)\right) \cong K_{1}(\mathbb{Z})$ and the image of $\eta_{*}: \pi_{j}(S) \rightarrow$ $K_{j}(\mathbb{Z})$ for $j \geq 2$ is described in $[67]$ and $[75]$. Let $R$ be any ring and for $i \geq 2$, let us write $X_{R}(i-1)$ for the $(i-1)$-connected cover of the 0-connected $K$-theory spectrum $X_{R}$. It is obvious that $\pi_{j}\left(X_{R}(i-1)\right) \cong K_{j}(R)$ for $j \geq i$. By Definition 6.2 and Proposition 6.4, there is an exact sequence

$$
\begin{aligned}
K_{i+2}(R) \stackrel{\bar{h}_{i+2}}{\longrightarrow} H_{i+2}\left(X_{R}(i-1) ; \mathbb{Z}\right) \stackrel{\bar{\nu}_{i+2}}{\longrightarrow} K_{i}(R) \otimes \pi_{1}(S) \\
\stackrel{\wedge}{\longrightarrow} K_{i+1}(R) \stackrel{\bar{h}_{i+1}}{\longrightarrow} H_{i+1}\left(X_{R}(i-1) ; \mathbb{Z}\right) \longrightarrow 0
\end{aligned}
$$

The diagram

$$
\begin{array}{ccc}
K_{i}(R) \otimes \pi_{1}(S) \stackrel{\wedge}{\longrightarrow} & K_{i+1}(R) \\
\operatorname{id} \otimes \eta_{*} \downarrow \cong & & \downarrow= \\
K_{i}(R) \otimes K_{1}(\mathbb{Z}) & \star & K_{i+1}(R),
\end{array}
$$

which commutes since $K_{R}(-1)$ is a $K_{\mathbb{Z}}(-1)$-module, shows that the above exact sequence is actually

$$
\begin{aligned}
K_{i+2}(R) \stackrel{\bar{h}_{i+2}}{\longrightarrow} H_{i+2}\left(X_{R}(i-1) ; \mathbb{Z}\right) \stackrel{\bar{\nu}_{i+2}}{\longrightarrow} K_{i}(R) \otimes K_{1}(\mathbb{Z}) \\
\stackrel{\star}{\longrightarrow} K_{i+1}(R) \stackrel{\bar{h}_{i+1}}{\longrightarrow} H_{i+1}\left(X_{R}(i-1) ; \mathbb{Z}\right) \longrightarrow 0 .
\end{aligned}
$$

Now, let us write $B G L(R)^{+}(i-1)$ for the $(i-1)$-connected cover of the infinite loop space $B G L(R)^{+}$and consider the commutative diagram

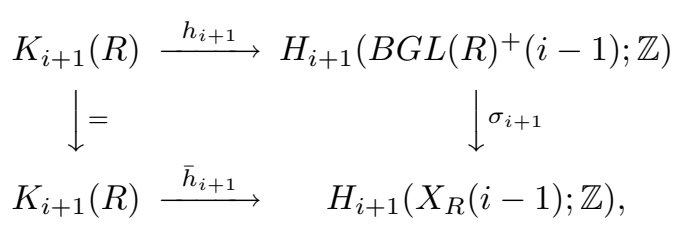

where the iterated homology suspension $\sigma_{i+1}$ is an isomorphism since $i \geq 2$ (see [100, Corollary VII.6.5]). Thus, the composition

$$
K_{i}(R) \otimes K_{1}(\mathbb{Z}) \stackrel{\star}{\longrightarrow} K_{i+1}(R) \stackrel{h_{i+1}}{\longrightarrow} H_{i+1}\left(B G L(R)^{+}(i-1) ; \mathbb{Z}\right)
$$


is trivial and the assertion immediately follows if one composes $h_{i+1}$ with the homomorphism

$$
H_{i+1}\left(B G L(R)^{+}(i-1) ; \mathbb{Z}\right) \rightarrow H_{i+1}\left(B G L(R)^{+} ; \mathbb{Z}\right) \cong H_{i+1}(G L(R) ; \mathbb{Z})
$$

induced by the obvious map $B G L(R)^{+}(i-1) \rightarrow B G L(R)^{+}$.

By an analogous argument, it is possible to generalize this result as follows.

Theorem 6.13. If $R$ is any ring, and if $i$ and $j$ are two integers such that $i-1 \geq j \geq 1$, then the composition

$$
K_{i}(R) \otimes K_{j}(\mathbb{Z}) \stackrel{\star}{\longrightarrow} K_{i+j}(R) \stackrel{h_{i+j}}{\longrightarrow} H_{i+j}(G L(R) ; \mathbb{Z})
$$

is trivial on all elements of the form $x \otimes y$ with $x \in K_{i}(R)$ and $y$ belonging to the image of $\eta_{*}: \pi_{j}(S) \rightarrow K_{j}(\mathbb{Z})$.

Proof: See [14, Proposition 3.1].

Remark 6.14. The assertions of Theorems 6.12 and 6.13 still hold if one replaces the infinite general linear group $G L(R)$ by the group of elementary matrices $E(R)$ or, if one assumes that $i \geq 3$, by the infinite Steinberg group $S t(R)$ (see [14, Proposition 3.1 and Theorem 3.2]).

In low dimensions, we are able to be more precise by providing exactness results. For instance, let us describe the unstable Hurewicz homomorphism in dimension 3 .

Theorem 6.15. For any ring $R$ there is a natural exact sequence

$$
K_{2}(R) \otimes K_{1}(\mathbb{Z}) \stackrel{\star}{\longrightarrow} K_{3}(R) \stackrel{h_{3}}{\longrightarrow} H_{3}(E(R) ; \mathbb{Z}) \longrightarrow 0 .
$$

Proof: (See [14, Theorem 4.1].) Let us consider the 1-connected infinite loop space $B E(R)^{+}$and kill all its homotopy groups above dimension 3 . We get its third Postnikov section (see also Section 7) $B E(R)^{+}[3]$ which has only two non-trivial homotopy groups $\pi_{2}\left(B E(R)^{+}[3]\right) \cong K_{2}(R)$ and $\pi_{3}\left(B E(R)^{+}[3]\right) \cong K_{3}(R)$. Therefore, $B E(R)^{+}[3]$ fits into the fibration of spaces

$$
K\left(K_{3}(R), 3\right) \longrightarrow B E(R)^{+}[3] \longrightarrow K\left(K_{2}(R), 2\right),
$$

in which the base space and the fiber are Eilenberg-MacLane spaces. Similarly, look at the third Postnikov section $X_{R}(1)[3]$ of the 1-connected cover $X_{R}(1)$ of $X_{R}$ and at the cofibration of spectra

$$
\Sigma^{3} H\left(K_{3}(R)\right) \longrightarrow X_{R}(1)[3] \longrightarrow \Sigma^{2} H\left(K_{2}(R)\right),
$$

in which the base and the fiber are Eilenberg-MacLane spectra. 
This induces the following commutative diagram where both columns are homology exact sequences:

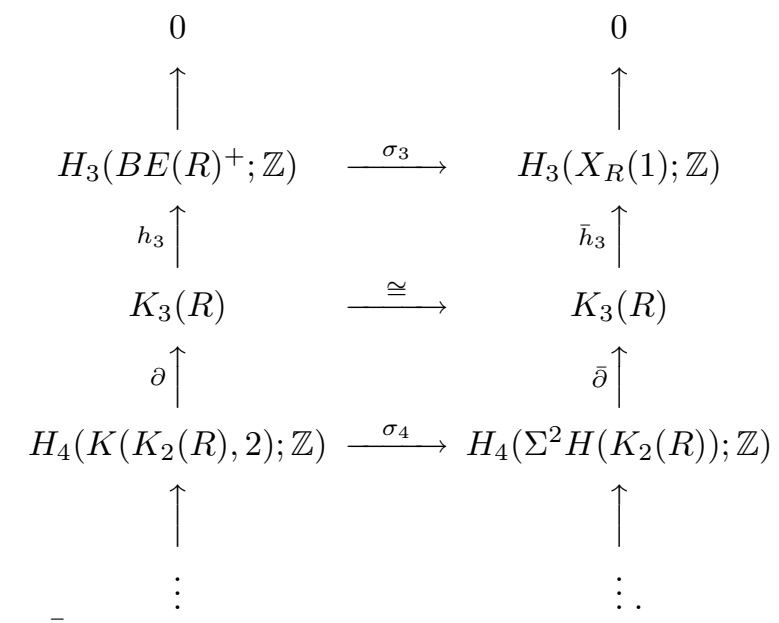

Here $\partial$ and $\bar{\partial}$ are connecting homomorphisms and the three horizontal arrows are iterated homology suspensions. Because of the long stable Whitehead exact sequence (see Definition 6.2 and Proposition 6.4), it turns out easily that

$$
\begin{aligned}
H_{4}\left(\Sigma^{2} H\left(K_{2}(R)\right) ; \mathbb{Z}\right) & \cong \pi_{3}\left(\Sigma^{2} H\left(K_{2}(R)\right) \wedge S(0)\right) \\
& \cong K_{2}(R) \otimes \pi_{1} S \cong K_{2}(R) \otimes K_{1}(\mathbb{Z})
\end{aligned}
$$

and it is again possible to check that $\bar{\partial}$ is the product $\star: K_{2}(R) \otimes$ $K_{1}(\mathbb{Z}) \rightarrow K_{3}(R)$ (see Proposition 2.2 of $[\mathbf{1 4}]$ ). Since $\sigma_{4}$ is surjective (see [100, Corollary VII.6.5]) one can deduce that the image of $\partial$ is actually equal to the image of $\bar{\partial}$, i.e., to the product $K_{2}(R) \star K_{1}(\mathbb{Z})$.

A similar argument provides the next theorem on the unstable Hurewicz homomorphism relating the algebraic $K$-theory of ring $R$ to the homology of its infinite Steinberg group in dimensions 4 and 5 .

Theorem 6.16. For any ring $R$ there is a natural exact sequence

$$
\begin{aligned}
K_{5}(R) \stackrel{h_{5}}{\longrightarrow} H_{5}(S t(R) ; \mathbb{Z}) & \longrightarrow K_{3}(R) \otimes K_{1}(\mathbb{Z}) \\
\stackrel{\star}{\longrightarrow} & K_{4}(R) \stackrel{h_{4}}{\longrightarrow} H_{4}(S t(R) ; \mathbb{Z}) \rightarrow 0
\end{aligned}
$$

and the kernel of $h_{5}$ fits into the natural exact sequence

$$
0 \longrightarrow K_{4}(R) \otimes K_{1}(\mathbb{Z}) \stackrel{\star}{\longrightarrow} \operatorname{ker} h_{5} \longrightarrow Q(R) \longrightarrow 0,
$$

where $Q(R)$ is a quotient of the subgroup of elements of order dividing 2 in $K_{3}(R)$. 
Proof: See [14, Theorem 4.3].

The last point of view from which we want to study the Hurewicz homomorphism is based on the Postnikov decomposition of $\mathrm{CW}$-complexes. This is the subject of the next section.

\section{The Postnikov invariants in algebraic $K$-theory}

The Postnikov invariants of a connected simple CW-complex $X$ are cohomology classes which provide the necessary information for the reconstruction of $X$, up to a weak homotopy equivalence, from its homotopy groups. Let $\alpha_{i}: X \rightarrow X[i]$ denote the $i$-th Postnikov section of $X$ for any positive integer $i$ : $X[i]$ is the CW-complex obtained from $X$ by killing the homotopy groups of $X$ in dimensions $>i$, more precisely by adjoining cells of dimensions $\geq i+2$ such that $\pi_{j}(X[i])=0$ for $j>i$ and $\left(\alpha_{i}\right)_{*}: \pi_{j}(X) \rightarrow \pi_{j}(X[i])$ is an isomorphism for $j \leq i$. Thus, we may view $X[i]$ as the $i$-th homotopical approximation of $X$. The Postnikov $k$-invariants of $X$ are cohomology classes

$$
k^{i+1}(X) \in H^{i+1}\left(X[i-1] ; \pi_{i}(X)\right),
$$

for $i \geq 2$, which are defined as follows (see for instance $[\mathbf{1 0 0}$, Section IX.2]).

Definition 7.1. Let $X$ be a simple CW-complex, $i$ an integer $\geq 2$, and let $\kappa^{i+1}$ denote the composition

$H_{i+1}(X[i-1], X[i] ; \mathbb{Z}) \stackrel{\left(\tilde{h}_{i+1}\right)^{-1}}{\longrightarrow} \pi_{i+1}(X[i-1], X[i]) \stackrel{\partial}{\longrightarrow} \pi_{i}(X[i]) \cong \pi_{i}(X)$,

where $\tilde{h}_{i+1}$ is the Hurewicz isomorphism for the $i$-connected pair $(X[i-1], X[i])$ and $\partial$ the connecting homomorphism (which is actually an isomorphism) of the homotopy exact sequence of that pair. Consider the isomorphism

$\lambda: \operatorname{Hom}\left(H_{i+1}(X[i-1], X[i] ; \mathbb{Z}), \pi_{i}(X)\right) \stackrel{\cong}{\longrightarrow} H^{i+1}\left(X[i-1], X[i] ; \pi_{i}(X)\right)$ given by the universal coefficient theorem and the homomorphism

$$
\nu: H^{i+1}\left(X[i-1], X[i] ; \pi_{i}(X)\right) \rightarrow H^{i+1}\left(X[i-1] ; \pi_{i}(X)\right)
$$

induced by the inclusion of pairs $(X[i-1], *) \hookrightarrow(X[i-1], X[i])$. The $k$-invariant $k^{i+1}(X)$ is defined by

$$
k^{i+1}(X)=\nu \lambda\left(\kappa^{i+1}\right) \in H^{i+1}\left(X[i-1] ; \pi_{i}(X)\right) .
$$


The main property of these invariants is that $X[i]$ is the homotopy fiber of the map $X[i-1] \rightarrow K\left(\pi_{i}(X), i+1\right)$ corresponding to the cohomology class $k^{i+1}(X) \in H^{i+1}\left(X[i-1] ; \pi_{i}(X)\right)$, for $i \geq 2$. In other words, there is a commutative diagram of fibrations

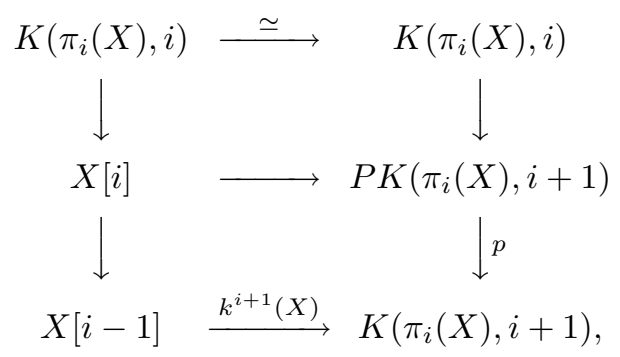

in which the right column is the path fibration over the EilenbergMacLane space $K\left(\pi_{i}(X), i+1\right)$ and the bottom square is a homotopy pull-back. Consequently, the knowledge of $X[i-1], \pi_{i}(X)$ and $k^{i+1}(X)$ enables us to construct the next homotopical approximation $X[i]$ of $X$.

Remark 7.2. From that point of view, the understanding of the (weak) homotopy type of the $K$-theory space $B G L(R)^{+}$of a ring $R$ depends on the knowledge of the $K$-groups $K_{i}(R)=\pi_{i}\left(B G L(R)^{+}\right)$and of the $k$-invariants $k^{i+1}\left(B G L(R)^{+}\right)$. In the remainder of this section and in Section 9 , we shall give some results on the $k$-invariants of $K$-theory spaces, especially on the (additive) order of the $k$-invariants $k^{i+1}\left(B G L(R)^{+}\right)$ considered as elements of the group $H^{i+1}\left(B G L(R)^{+}[i-1] ; K_{i}(R)\right)$.

In order to understand the role of the $k$-invariants of a simple $\mathrm{CW}$-complex, let us first mention the following obvious fact.

Lemma 7.3. If $k^{i+1}(X)=0$ in $H^{i+1}\left(X[i-1] ; \pi_{i}(X)\right)$, then

$$
X[i] \simeq X[i-1] \times K\left(\pi_{i}(X), i\right)
$$

and the Hurewicz homomorphism $h_{i}: \pi_{i}(X) \rightarrow H_{i}(X ; \mathbb{Z})$ is split injective.

Proof: Since the diagram occuring in Definition 7.1 is a pull-back, the vanishing of $k^{i+1}(X)$ implies that

$$
\begin{aligned}
X[i] & =\left\{(x, y) \in X[i-1] \times P K\left(\pi_{i}(X), i+1\right) \mid p(y)=*\right\} \\
& \simeq X[i-1] \times(\text { fiber of } p) \simeq X[i-1] \times K\left(\pi_{i}(X), i\right) .
\end{aligned}
$$


By definition of $\alpha_{i}: X \rightarrow X[i]$, the induced homomorphism $\left(\alpha_{i}\right)_{*}$ : $H_{j}(X ; \mathbb{Z}) \rightarrow H_{j}(X[i] ; \mathbb{Z})$ is an isomorphism for $j \leq i$ by the Whitehead theorem (see [100, Theorem IV.7.13]). Thus, the Künneth formula gives

$$
H_{i}(X ; \mathbb{Z}) \cong H_{i}(X[i] ; \mathbb{Z}) \cong H_{i}(X[i-1] ; \mathbb{Z}) \oplus \pi_{i}(X) .
$$

One of the crucial properties of the $k$-invariants is the following lemma which follows almost directly from Definition 7.1 (see [100, Section IX.5, Example 3]).

Lemma 7.4. If $X$ is a loop space $X \simeq \Omega Y$, then the $k$-invariants of $X$ and $Y$ are related by the formula $\sigma^{*}\left(k^{i+2}(Y)\right)=k^{i+1}(X)$, where $\sigma^{*}: H^{i+2}\left(Y[i] ; \pi_{i}(X)\right) \rightarrow H^{i+1}\left(X[i-1] ; \pi_{i}(X)\right)$ is the cohomology suspension.

Our first result is a vanishing theorem (see Theorem 7.6 below) based on the following remark on the cohomology suspension for EilenbergMacLane spaces.

Proposition 7.5. For any abelian groups $G$ and $M$, the double cohomology suspension

$$
\left(\sigma^{*}\right)^{2}: H^{5}(K(G, 3) ; M) \longrightarrow H^{4}(K(G, 2) ; M) \longrightarrow H^{3}(K(G, 1) ; M)
$$

is trivial.

Proof: For any abelian group $G$, it is known that $H_{4}(K(G, 3) ; \mathbb{Z})=0$ and it follows easily from Remark 6.3 that $H_{5}(K(G, 3) ; \mathbb{Z}) \cong \Gamma_{4}(K(G, 3)) \cong$ $G \otimes \pi_{1}(S)=G \otimes \mathbb{Z} / 2 \cong G / 2 G$ (see also [100, Theorems V.7.8 and XII.3.20]). Thus, the universal coefficient theorem provides an isomorphism

$$
H^{5}(K(G, 3) ; M) \cong \operatorname{Hom}(G / 2 G, M) .
$$

For any element $u \in H^{5}(K(G, 3) ; M)$ let us write $\widehat{u}$ for the corresponding element in $\operatorname{Hom}(G / 2 G, M)$. For example, if one takes any abelian group $G$ and $M=G / 2 G$, the element $\widehat{S q^{2}}$ corresponding to the Steenrod square $S q^{2}$ viewed as a cohomology operation belonging to $H^{5}(K(G, 3) ; G / 2 G)$ turns out to be the identity id $\in \operatorname{Hom}(G / 2 G, G / 2 G)$. Now, for any cohomology class $u \in H^{5}(K(G, 3) ; M)$, it is clear that $\widehat{u}=\widehat{u}_{\sharp}(\mathrm{id})=\widehat{u}_{\sharp}\left(\widehat{S q^{2}}\right)$, where $\widehat{u}_{\sharp}: \operatorname{Hom}(G / 2 G, G / 2 G) \rightarrow \operatorname{Hom}(G / 2 G, M)$ is induced by $\widehat{u} \in \operatorname{Hom}(G / 2 G, M)$. Consequently, $u=\widehat{u}_{*}\left(S q^{2}\right)$, where 
$\widehat{u}_{*}: H^{5}(K(G, 3) ; G / 2 G) \rightarrow H^{5}(K(G, 3) ; M)$ is the homomorphism induced by $\widehat{u}$. Finally, let us consider the commutative diagram

$$
\begin{array}{ccc}
H^{5}(K(G, 3) ; G / 2 G) & \stackrel{\widehat{u}_{*}}{\longrightarrow} H^{5}(K(G, 3) ; M) \\
\downarrow\left(\sigma^{*}\right)^{2} & & \downarrow\left(\sigma^{*}\right)^{2} \\
H^{3}(K(G, 1) ; G / 2 G) \stackrel{\widehat{u}_{*}}{\longrightarrow} & H^{3}(K(G, 1) ; M) .
\end{array}
$$

Because it is well known that the cohomology operation $\left(\sigma^{*}\right)^{2}\left(S q^{2}\right)$ is trivial in $H^{3}(K(G, 1) ; G / 2 G)$, we may deduce that

$$
\left(\sigma^{*}\right)^{2}(u)=\left(\sigma^{*}\right)^{2}\left(\widehat{u}_{*}\left(S q^{2}\right)\right)=\widehat{u}_{*}\left(\sigma^{*}\right)^{2}\left(S q^{2}\right)=0 .
$$

Theorem 7.6. The first $k$-invariant $k^{3}(X) \in H^{3}\left(K\left(\pi_{1}(X), 1\right) ; \pi_{2}(X)\right)$ of any connected double loop space $X$ is trivial.

Proof: Consider any connected double loop space $X \simeq \Omega^{2} Y$. We may assume that $Y$ is 2-connected and consequently that $Y[3] \simeq K\left(\pi_{3}(Y), 3\right) \simeq$ $K\left(\pi_{1}(X), 3\right)$. According to Lemma $7.4, k^{3}(X)=\left(\sigma^{*}\right)^{2}\left(k^{5}(Y)\right)$, where $\left(\sigma^{*}\right)^{2}$ is the double cohomology suspension

$$
\begin{aligned}
\left(\sigma^{*}\right)^{2}: H^{5}(Y[3] ; & \left.\pi_{2}(X)\right) \cong H^{5}\left(K\left(\pi_{1}(X), 3\right) ; \pi_{2}(X)\right) \\
& \longrightarrow H^{3}\left(X[1] ; \pi_{2}(X)\right) \cong H^{3}\left(K\left(\pi_{1}(X), 1\right) ; \pi_{2}(X)\right) .
\end{aligned}
$$

Therefore, the assertion is a direct consequence of Proposition 7.5. See [8] for another proof.

Corollary 7.7. For any connected double loop space $X$,

$$
H_{2}(X ; \mathbb{Z}) \cong \pi_{2}(X) \oplus \Lambda^{2}\left(\pi_{1}(X)\right)
$$

where $\Lambda^{2}$ denotes the exterior square.

Proof: Since $k^{3}(X)=0$ in $H^{3}\left(K\left(\pi_{1}(X), 1\right) ; \pi_{2}(X)\right)$ by the previous theorem, the second Postnikov section $X[2]$ of $X$ is a product of EilenbergMacLane spaces according to Lemma 7.3:

$$
X[2] \simeq K\left(\pi_{1}(X), 1\right) \times K\left(\pi_{2}(X), 2\right) .
$$

Thus,

$$
H_{2}(X ; \mathbb{Z}) \cong H_{2}\left(K\left(\pi_{1}(X), 1\right) ; \mathbb{Z}\right) \oplus H_{2}\left(K\left(\pi_{2}(X), 2\right) ; \mathbb{Z}\right) .
$$

The second summand is isomorphic to $\pi_{2}(X)$ by the Hurewicz theorem and the fact that $X$ is an $H$-space implies that $\pi_{1}(X)$ is abelian and consequently that $H_{2}\left(K\left(\pi_{1}(X), 1\right) ; \mathbb{Z}\right) \cong \Lambda^{2}\left(\pi_{1}(X)\right)$ (see $[\mathbf{3 7}$, Theorem V.6.4]). 
A direct application of that result to the infinite loop space $B G L(R)^{+}$ (see Remark 4.12) provides the following splitting (see also [11, Section 3], for the discussion of the naturality of that splitting).

Theorem 7.8. For any ring $R$,

$$
H_{2}(G L(R) ; \mathbb{Z}) \cong K_{2}(R) \oplus \Lambda^{2}\left(K_{1}(R)\right) .
$$

Remark 7.9. This statement is quite obvious when the ring $R$ is commutative with $S K_{1}(R)=0$. In that case, $E(R)=S L(R), B S L(R)^{+} \simeq$ $B \widetilde{G L(R)^{+}}, K_{1}(R)=R^{\times}$(see Theorem 2.9 and Lemma 3.3), and there is a fibration of infinite loop spaces $B S L(R)^{+} \rightarrow B G L(R)^{+} \rightarrow K\left(R^{\times}, 1\right)$ which has a splitting induced by the inclusion $R^{\times}=G L_{1}(R) \hookrightarrow G L(R)$. Therefore, $B G L(R)^{+} \simeq B S L(R)^{+} \times K\left(R^{\times}, 1\right)$ and one gets the assertion. However, in the general case, the above topological argument involving $k^{3}\left(B G L(R)^{+}\right)$is necessary.

This kind of nice consequences can be generalized when the $k$-invariant $k^{i+1}(X)$ is a cohomology class which is not trivial, but of finite order in the group $H^{i+1}\left(X[i-1] ; \pi_{i}(X)\right)$.

Proposition 7.10. Let $X$ be a connected simple $C W$-complex, $i$ an integer $\geq 2$ and $\rho$ a positive integer. The following assertions are equivalent:

(a) $\rho k^{i+1}(X)=0$ in $H^{i+1}\left(X[i-1] ; \pi_{i}(X)\right)$.

(b) There is a map

$$
f_{i}: X \longrightarrow K\left(\pi_{i}(X), i\right)
$$

such that the induced homomorphism $\left(f_{i}\right)_{*}: \pi_{i}(X) \rightarrow \pi_{i}(X)$ is multiplication by $\rho$.

(c) There is a homomorphism $\theta_{i}: H_{i}(X ; \mathbb{Z}) \rightarrow \pi_{i}(X)$ such that the composition

$$
\pi_{i}(X) \stackrel{h_{i}}{\longrightarrow} H_{i}(X ; \mathbb{Z}) \stackrel{\theta_{i}}{\longrightarrow} \pi_{i}(X)
$$

is multiplication by $\rho$.

Proof: (See also Section 1 of [16].) If (a) holds, the composition

$$
X[i-1] \stackrel{k^{i+1}(X)}{\longrightarrow} K\left(\pi_{i}(X), i+1\right) \stackrel{\rho(\mathrm{id})}{\longrightarrow} K\left(\pi_{i}(X), i+1\right)
$$

(where id is written for the identity $K\left(\pi_{i}(X), i+1\right) \rightarrow K\left(\pi_{i}(X), i+1\right)$ ) is trivial since it corresponds to the cohomology class $\rho k^{i+1}(X)=0$. 
Therefore, we have the following commutative diagram

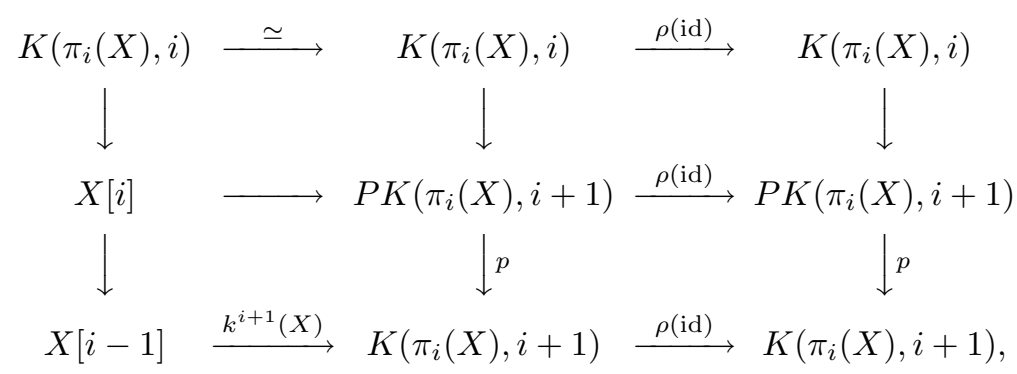

where all columns are fibrations and in which the bottom left square is a pull-back by definition of the $k$-invariant $k^{i+1}(X)$. Let $E$ be the pull-back of $\left(\rho k^{i+1}(X), p\right)$. Since the bottom composition in the above diagram is nullhomotopic, $E$ is a product $E \simeq X[i-1] \times K\left(\pi_{i}(X), i\right)$. Since $E$ is a pull-back, there is a map $\varphi: X[i] \rightarrow E$ inducing an isomorphism on $\pi_{j}$ for $j \leq i-1$ and multiplication by $\rho$ on $\pi_{i}$. Thus, we can define

$$
f_{i}: X \stackrel{\alpha_{i}}{\longrightarrow} X[i] \stackrel{\varphi}{\longrightarrow} E \simeq X[i-1] \times K\left(\pi_{i}(X), i\right) \longrightarrow K\left(\pi_{i}(X), i\right),
$$

where the last map is the projection onto the second factor. This map induces multiplication by $\rho$ on the only interesting homotopy group $\pi_{i}$ :

$$
\left(f_{i}\right)_{*}: \pi_{i}(X) \stackrel{\cdot \rho}{\longrightarrow} \pi_{i}(X) .
$$

Assertion (c) follows from (b) because of the commutativity of the diagram

$$
\begin{array}{ccc}
\pi_{i}(X) & \stackrel{\left(f_{i}\right)_{*}}{\longrightarrow} & \pi_{i}(X) \\
& & \downarrow \cong \\
h_{i} & & \\
H_{i}(X ; \mathbb{Z}) \stackrel{\left(f_{i}\right)_{*}}{\longrightarrow} & H_{i}\left(K\left(\pi_{i}(X), i\right) ; \mathbb{Z}\right) \cong \pi_{i}(X)
\end{array}
$$

induced by the map $f_{i}$, where both vertical arrows are Hurewicz homomorphisms: we call $\theta_{i}$ the bottom horizontal homomorphism $\left(f_{i}\right)_{*}$ in that diagram.

In order to prove that (a) follows from (c), let us look at the commutative diagram

$$
\begin{aligned}
& \pi_{i+1}(X[i-1], X[i]) \stackrel{\tilde{h}_{i+1}}{\cong} H_{i+1}(X[i-1], X[i] ; \mathbb{Z}) \\
& \cong \downarrow \partial \quad \downarrow \widetilde{\partial} \\
& \pi_{i}(X) \cong \pi_{i}(X[i]) \stackrel{h_{i}}{\longrightarrow} H_{i}(X[i] ; \mathbb{Z}) \cong H_{i}(X ; \mathbb{Z}),
\end{aligned}
$$


in which the horizontal arrows are Hurewicz homomorphisms and the vertical arrows are connecting homomorphisms. If $\theta_{i}: H_{i}(X ; \mathbb{Z}) \rightarrow \pi_{i}(X)$ exists as in (c), we deduce that

$$
\theta_{i} \widetilde{\partial}=\theta_{i} h_{i} \partial\left(\tilde{h}_{i+1}\right)^{-1}=\rho \partial\left(\tilde{h}_{i+1}\right)^{-1}=\rho \kappa^{i+1},
$$

where $\kappa^{i+1}$ is the element introduced in Definition 7.1. Thus, the image of $\rho \kappa^{i+1}$ under the isomorphism

$\lambda: \operatorname{Hom}\left(H_{i+1}(X[i-1], X[i] ; \mathbb{Z}), \pi_{i}(X)\right) \stackrel{\cong}{\longrightarrow} H^{i+1}\left(X[i-1], X[i] ; \pi_{i}(X)\right)$ belongs to the image of the connecting homomorphism

$$
\delta: H^{i}\left(X[i] ; \pi_{i}(X)\right) \rightarrow H^{i+1}\left(X[i-1], X[i] ; \pi_{i}(X)\right) .
$$

The exactness of the cohomology sequence

$$
\begin{aligned}
H^{i}\left(X[i] ; \pi_{i}(X)\right) \stackrel{\delta}{\longrightarrow} H^{i+1}(X[i-1], X[i] & \left.; \pi_{i}(X)\right) \\
& \stackrel{\nu}{\longrightarrow} H^{i+1}\left(X[i-1] ; \pi_{i}(X)\right)
\end{aligned}
$$

of the pair $(X[i-1], X[i])$ finally implies that $\rho k^{i+1}=\rho \nu \lambda\left(\kappa^{i+1}\right)=$ $\nu \lambda\left(\rho \kappa^{i+1}\right)=0$.

Because of these equivalences, it is really important to prove finiteness results for the order of the $k$-invariants in algebraic $K$-theory. For that purpose, we first need to recall that $\mathrm{H}$. Cartan computed the homology of Eilenberg-MacLane spaces in [38]; in particular, according to his calculation (see [38, Théorème 2]), the stable homotopy groups of Eilenberg-MacLane spaces have a quite small exponent. This can be formulated as follows.

Definition 7.11. Let $L_{1}:=1$, and for $k \geq 2$ let $L_{k}$ denote the product of all primes $p$ for which there exists a sequence of non-negative integers $\left(a_{1}, a_{2}, a_{3}, \ldots\right)$ satisfying:

(a) $a_{1} \equiv 0 \bmod (2 p-2), a_{i} \equiv 0$ or $1 \bmod (2 p-2)$ for $i \geq 2$,

(b) $a_{i} \geq p a_{i+1}$ for $i \geq 1$,

(c) $\sum_{i=1}^{\infty} a_{i}=k$.

For example, $L_{2}=2, L_{3}=2, L_{4}=6, L_{5}=6, L_{6}=2, L_{7}=2$, $L_{8}=30, \ldots$. Observe that $L_{k}$ divides the product of all primes $p \leq \frac{k}{2}+1$.

Lemma 7.12. For any abelian group $G$ and any pair of integers $i$ and $m$ with $2 \leq m<i<2 m$, one has $L_{i-m} H_{i}(K(G, m) ; \mathbb{Z})=0$.

Proof: This follows directly from Cartan's determination of the stable homology of Eilenberg-MacLane spaces given by Théorème 2 of [38].

This implies the following consequence. 
Corollary 7.13. Let $X$ be $a(b-1)$-connected $C W$-complex (with $b \geq 2$ ) such that there exists an integer $t \geq b$ with the property that $\pi_{i}(X)=0$ for $i>t$ (in other words, such that $X=X[t]$ ). Then

$$
L_{i-b} L_{i-b-1} L_{i-b-2} \cdots L_{i-t} H_{i}(X ; \mathbb{Z})=0
$$

if $t<i<2 b$.

Proof: Let $i$ be an integer such that $t<i<2 b$. If $t=b$, then $X$ is an Eilenberg-MacLane space $X=K\left(\pi_{b}(X), b\right)$ and the result is given by Lemma 7.12. Now, let us suppose $t>b$. For any integer $k$ with $1 \leq k \leq t-b$, let us consider the fibration

$$
K\left(\pi_{b+k}(X), b+k\right) \longrightarrow X[b+k] \longrightarrow X[b+k-1],
$$

whose Serre spectral sequence provides the exact sequence

$$
H_{i}\left(K\left(\pi_{b+k}(X), b+k\right) ; \mathbb{Z}\right) \longrightarrow H_{i}(X[b+k] ; \mathbb{Z}) \longrightarrow H_{i}(X[b+k-1] ; \mathbb{Z}),
$$

since $i<2 b$. Observe that for $k=1, X[b+k-1]=X[b]=K\left(\pi_{b}(X), b\right)$ and consequently that $L_{i-b} H_{i}(X[b] ; \mathbb{Z})=0$ according to Lemma 7.12. For the same reason, $L_{i-b-k} H_{i}\left(K\left(\pi_{b+k}(X), b+k\right) ; \mathbb{Z}\right)=0$ for $1 \leq k \leq t-b$. We then conclude by induction that $L_{i-b} L_{i-b-1} L_{i-b-2} \cdots L_{i-t} H_{i}(X[t] ; \mathbb{Z})=$ 0 and get the assertion because $X[t]=X$ by hypothesis.

Definition 7.14. Let $R_{j}:=1$ for $j \leq 1$ and $R_{j}:=\prod_{k=2}^{j} L_{k}$ for $j \geq 2$. For example, $R_{2}=2, R_{3}=4, R_{4}=24, R_{5}=144, R_{6}=288 R_{7}=576$, $R_{8}=17280, \ldots$. It turns out that a prime number $p$ divides $R_{j}$ if and only if $p \leq \frac{j}{2}+1$.

This definition enables us to describe universal bounds for the order of the $k$-invariants of iterated loop spaces (see $[\mathbf{6}]$ and Section 1 of $[\mathbf{1 0}]$ ). Let us emphasize the fact that the next result holds without any finiteness condition on the space we are looking at.

Theorem 7.15. If $X$ is $a(b-1)$-connected $r$-fold loop space (with $b \geq 1$, $r \geq 0)$, i.e., $X \simeq \Omega^{r} Y$ for some $(b+r-1)$-connected $C W$-complex $Y$, then

$$
R_{i-b+1} k^{i+1}(X)=0 \quad \text { in } \quad H^{i+1}\left(X[i-1] ; \pi_{i}(X)\right)
$$

for all integers $i$ such that $2 \leq i \leq r+2 b-2$.

Proof: Since $X$ is $(b-1)$-connected, it is clear that $k^{i+1}(X)=0$ for $2 \leq i \leq b$. Thus, we may assume that $b+1 \leq i \leq r+2 b-2$, in particular that $r+b \geq 3$. It follows from the homotopy equivalence $X \simeq \Omega^{r} Y$ that 
$\pi_{i}(X) \cong \pi_{i+r}(Y)$ and from Lemma 7.4 that the iterated cohomology suspension

$$
\left(\sigma^{*}\right)^{r}: H^{i+r+1}\left(Y[i+r-1] ; \pi_{i}(X)\right) \longrightarrow H^{i+1}\left(X[i-1] ; \pi_{i}(X)\right)
$$

satisfies

$$
\left(\sigma^{*}\right)^{r}\left(k^{i+r+1}(Y)\right)=k^{i+1}(X) .
$$

Since we may assume that $Y$ is $(b+r-1)$-connected, we deduce from Corollary 7.13 that

$$
L_{j-b-r} L_{j-b-r-1} L_{j-b-r-2} \cdots L_{j-i-r+1} H_{j}(Y[i+r-1] ; \mathbb{Z})=0
$$

for $i+r-1<j<2 b+2 r$, in particular for $j=i+r$ and $j=i+r+1$ :

$$
\begin{gathered}
\underbrace{L_{i-b} L_{i-b-1} L_{i-b-2} \cdots L_{1}}_{=R_{i-b}} H_{i+r}(Y[i+r-1] ; \mathbb{Z})=0, \\
\underbrace{L_{i-b+1} L_{i-b} L_{i-b-1} \cdots L_{2}}_{=R_{i-b+1}} H_{i+r+1}(Y[i+r-1] ; \mathbb{Z})=0 .
\end{gathered}
$$

Therefore, the universal coefficient theorem shows that the exponent of the group $H^{i+r+1}\left(Y[i+r-1] ; \pi_{i}(X)\right)$ is bounded by $\operatorname{lcm}\left(R_{i-b}, R_{i-b+1}\right)=$ $R_{i-b+1}$. Thus, $R_{i-b+1} k^{i+r+1}(Y)=0$ and

$$
\begin{aligned}
R_{i-b+1} k^{i+1}(X) & =R_{i-b+1}\left(\sigma^{*}\right)^{r}\left(k^{i+r+1}(Y)\right) \\
& =\left(\sigma^{*}\right)^{r}\left(R_{i-b+1} k^{i+r+1}(Y)\right)=0 .
\end{aligned}
$$

Corollary 7.16. For any $(b-1)$-connected infinite loop space $X$ (with $b \geq 1)$

$$
R_{i-b+1} k^{i+1}(X)=0 \quad \text { in } \quad H^{i+1}\left(X[i-1] ; \pi_{i}(X)\right)
$$

for all integers $i \geq 2$.

In the case of the $K$-theory spaces, we get the following result.

Theorem 7.17. For any ring $R$,

(a) $R_{i} k^{i+1}\left(B G L(R)^{+}\right)=0$ for all $i \geq 2$,

(b) $R_{i-1} k^{i+1}\left(B E(R)^{+}\right)=0$ for all $i \geq 3$,

(c) $R_{i-2} k^{i+1}\left(B S t(R)^{+}\right)=0$ for all $i \geq 4$.

Proof: This follows from Corollary 7.16, because $B G L(R)^{+}, B E(R)^{+}$ and $B S t(R)^{+}$are infinite loop spaces which are connected, simply connected and 2-connected respectively. 
Let us look at immediate consequences of this theorem for the Hurewicz homomorphism relating the $K$-groups of any ring $R$ to the homology of the infinite general linear group over $R$, respectively of the infinite special linear group and of the infinite Steinberg group. Remember that this homomorphism is an isomorphism in the first non-trivial dimension (see Theorem 6.1). Our next result approximates the exponent of the kernel of the Hurewicz homomorphism in all dimensions (see also [9]).

Remark 7.18. In [87, Proposition 3], C. Soulé has shown that the kernel of $h_{i}: K_{i}(R) \rightarrow H_{i}(E(R) ; \mathbb{Z})$ is a torsion group that involves only prime numbers $p$ satisfying $p \leq \frac{i+1}{2}$, but his argument does not imply that this kernel has finite exponent.

Corollary 7.19. Let $R$ be any ring.

(a) For any $i \geq 2$, the Hurewicz homomorphism

satisfies $R_{i}\left(\operatorname{ker} h_{i}\right)=0$.

$$
h_{i}: K_{i}(R) \rightarrow H_{i}(G L(R) ; \mathbb{Z})
$$

(b) For any $i \geq 3$, the Hurewicz homomorphism

$$
h_{i}: K_{i}(R) \rightarrow H_{i}(E(R) ; \mathbb{Z})
$$

satisfies $R_{i-1}\left(\operatorname{ker} h_{i}\right)=0$.

(c) For any $i \geq 4$, the Hurewicz homomorphism

$$
h_{i}: K_{i}(R) \rightarrow H_{i}(S t(R) ; \mathbb{Z})
$$

satisfies $R_{i-2}\left(\operatorname{ker} h_{i}\right)=0$.

Proof: Let us start with the 0-connected infinite loop space $B G L(R)^{+}$. Because of Proposition 7.10 and of Corollary 7.16, there is a homomorphism $\theta_{i}: H_{i}(G L(R) ; \mathbb{Z}) \cong H_{i}\left(B G L(R)^{+} ; \mathbb{Z}\right) \rightarrow K_{i}(R)$ such that the composition

$$
K_{i}(R) \stackrel{h_{i}}{\longrightarrow} H_{i}(G L(R) ; \mathbb{Z}) \stackrel{\theta_{i}}{\longrightarrow} K_{i}(R)
$$

is multiplication by $R_{i}$. If $x$ belongs to the kernel of $h_{i}$, then $R_{i} x=$ $\theta_{i} h_{i}(X)=0$. The same argument works for $B E(R)^{+}$and $B S t(R)^{+}$.

Remark 7.20. Of course, the assertion (a) is less interesting than the other ones since it can be improved: for instance, in the case where $i=2$, we know from Theorem 7.8 that $h_{2}: K_{2}(R) \rightarrow H_{2}(G L(R) ; \mathbb{Z})$ is split injective for any ring $R$.

Example 7.21. Corollary 7.19 shows that $h_{3}: K_{3}(R) \rightarrow H_{3}(E(R) ; \mathbb{Z})$ fulfills

$$
2\left(\operatorname{ker} h_{3}\right)=0
$$


for any ring $R$. This was first observed by A. A. Suslin in [91, Proof of Proposition 4.5] (no details are given there). Later, C. H. Sah has also established that 2 ker $h_{3}=0$ for any ring $A$ (see [80, Proposition 2.5]), but unfortunately, there is a gap in his proof (see [9, Remark 1.9]).

Corollary 7.16 also provides another proof of Corollary 6.11.

Corollary 7.22. Let $R$ be any ring.

(a) For any integer $i \geq 1$, the Hurewicz homomorphism

$$
h_{i}: K_{i}\left(R ; \mathbb{Z}_{(p)}\right) \rightarrow H_{i}\left(G L(R) ; \mathbb{Z}_{(p)}\right)
$$

is a split injection for all primes $p \geq \frac{i+3}{2}$.

(b) For any integer $i \geq 2$, the Hurewicz homomorphism

$$
h_{i}: K_{i}\left(R ; \mathbb{Z}_{(p)}\right) \rightarrow H_{i}\left(E(R) ; \mathbb{Z}_{(p)}\right)
$$

is a split injection for all primes $p \geq \frac{i+2}{2}$.

(c) For any integer $i \geq 3$, the Hurewicz homomorphism

$$
h_{i}: K_{i}\left(R ; \mathbb{Z}_{(p)}\right) \rightarrow H_{i}\left(S t(R) ; \mathbb{Z}_{(p)}\right)
$$

is a split injection for all primes $p \geq \frac{i+1}{2}$.

Proof: Let us look again at the composition

$$
K_{i}(R) \stackrel{h_{i}}{\longrightarrow} H_{i}(G L(R) ; \mathbb{Z}) \stackrel{\theta_{i}}{\longrightarrow} K_{i}(R)
$$

which is multiplication by $R_{i}$. Since $R_{i}$ is only divisible by primes $p \leq \frac{i+2}{2}$, the composition

$$
K_{i}\left(R ; \mathbb{Z}_{(p)}\right) \stackrel{h_{i}}{\longrightarrow} H_{i}\left(G L(R) ; \mathbb{Z}_{(p)}\right) \stackrel{\theta_{i}}{\longrightarrow} K_{i}\left(R ; \mathbb{Z}_{(p)}\right)
$$

is an isomorphism and $h_{i}$ is a split injection when $p \geq \frac{i+3}{2}$. The proof is analogous for the simply connected infinite loop space $B E(R)^{+}$(with $p$ dividing $R_{i-1}$ if and only if $p \leq \frac{i+1}{2}$ ) and for the 2-connected infinite loop space $B S t(R)^{+}$(with $p$ dividing $R_{i-2}$ if and only if $p \leq \frac{i}{2}$ ).

Let us conclude this section by mentioning a result on the homotopy type of the $K$-theory space of algebraically closed fields (see also [9, Theorem 2.4]).

Theorem 7.23. Let $F$ be an algebraically closed field and $i$ any positive even integer. Then,

(a) the Postnikov $k$-invariant $k^{i+1}\left(B S L(F)^{+}\right)$is trivial in $H^{i+1}\left(B S L(F)^{+}[i-1] ; K_{i}(F)\right)$,

(b) the Hurewicz homomorphism $h_{i}: K_{i}(F) \rightarrow H_{i}(S L(F) ; \mathbb{Z})$ is split injective. 
Proof: Since $B S L(F)^{+}$is a simply connected infinite loop space, we may consider an $(i-1)$-connected space $Y$ with $B S L(F)^{+} \simeq \Omega^{i-2} Y$. By Lemma 7.4, the $k$-invariant $k^{i+1}\left(B S L(F)^{+}\right)$is then the image of $k^{2 i-1}(Y)$ under the $(i-2)$-fold iterated cohomology suspension

$$
\left(\sigma^{*}\right)^{i-2}: H^{2 i-1}\left(Y[2 i-3] ; K_{i}(F)\right) \rightarrow H^{i+1}\left(B S L(F)^{+}[i-1] ; K_{i}(F)\right) .
$$

Now, look at the universal coefficient theorem

$$
\begin{aligned}
& H^{2 i-1}\left(Y[2 i-3] ; K_{i}(F)\right) \cong \operatorname{Hom}(\left.H_{2 i-1}(Y[2 i-3] ; \mathbb{Z}), K_{i}(F)\right) \\
& \oplus \operatorname{Ext}\left(H_{2 i-2}(Y[2 i-3] ; \mathbb{Z}), K_{i}(F)\right),
\end{aligned}
$$

and observe that the group $\operatorname{Ext}\left(H_{2 i-2}(Y[2 i-3] ; \mathbb{Z}), K_{i}(F)\right)$ vanishes because A. A. Suslin proved in Section 2 of $[\mathbf{9 2}]$ that $K_{i}(F)$ is divisible for algebraically closed fields. Moreover, he also obtained in [92, Section 2], that $K_{i}(F)$ is torsion-free if $i$ is an even integer: this and the fact that $H_{2 i-1}(Y[2 i-3] ; \mathbb{Z})$ is a torsion group (see Corollary 7.13) imply that $\operatorname{Hom}\left(H_{2 i-1}(Y[2 i-3] ; \mathbb{Z}), K_{i}(F)\right)$ is trivial. Consequently, $k^{i+1}\left(B S L(F)^{+}\right)=\left(\sigma^{*}\right)^{i-2}\left(k^{2 i-1}(Y)\right)$ vanishes because $k^{2 i-1}(Y) \in$ $H^{2 i-1}\left(Y[2 i-3] ; K_{i}(F)\right)=0$. Assertion (b) follows from Lemma 7.3.

\section{The algebraic $K$-theory of number fields and rings of integers}

In the remainder of the paper, let us concentrate our attention on a specific class of rings: we want to investigate the $K$-groups of number fields and rings of integers. This plays an important role because of the various interactions between algebraic $K$-theory and number theory. Let $F$ be a number field (i.e., a finite extension of the field of rationals $\mathbb{Q}$ ) and $O_{F}$ its ring of algebraic integers. D. Quillen obtained in 1973 the first result on the structure of the groups $K_{i}\left(O_{F}\right)$ (see [73, Theorem 1]).

Theorem 8.1 (Quillen). For any number field $F$ and for any integer $i \geq 0, K_{i}\left(O_{F}\right)$ is a finitely generated abelian group.

The corresponding result does not hold for the number field $F$ itself: the structure of the abelian groups $K_{i}(F)$ is much more complicated, and consequently much more interesting. Of course, the groups $K_{i}(F)$ and $K_{i}\left(O_{F}\right)$ are strongly related. In order to observe that relation, D. Quillen constructed in Sections 5 and 7 of [72] (see also [74, Theorem 4]) a fibration

$$
\prod_{\mathfrak{m}} B Q \mathcal{P}\left(O_{F} / \mathfrak{m}\right) \longrightarrow B Q \mathcal{P}\left(O_{F}\right) \longrightarrow B Q \mathcal{P}(F)
$$


where $\prod$ is the weak product (i.e., the direct limit of cartesian products with finitely many factors), where $\mathfrak{m}$ runs over the set of all maximal ideals of $O_{F}$ and where the last map is induced by the inclusion $O_{F} \hookrightarrow F$. Here, for any ring $R, \mathcal{P}(R)$ is the category of finitely generated projective $R$-modules and $B Q \mathcal{P}(-)$ denotes the $Q$-construction mentioned in Remark 3.16: in particular, its loop space fulfills the homotopy equivalence $\Omega B Q \mathcal{P}(R) \simeq B G L(R)^{+} \times K_{0}(R)$. By looping the base space and the total space of the above fibration and by taking the 0 -connected covers of the the three spaces, we get the fibration

$$
B G L\left(O_{F}\right)^{+} \longrightarrow B G L(F)^{+} \longrightarrow \prod_{\mathfrak{m}} \Omega^{-1} B G L\left(O_{F} / \mathfrak{m}\right)^{+} .
$$

The homotopy exact sequence of that fibration provides the following long exact sequence.

Theorem 8.2. For any number field $F$, there is a long exact sequence (called the localization sequence in algebraic $K$-theory)

$$
\begin{aligned}
& \cdots \longrightarrow K_{i}\left(O_{F}\right) \longrightarrow K_{i}(F) \longrightarrow \bigoplus_{\mathfrak{m}} K_{i-1}\left(O_{F} / \mathfrak{m}\right) \longrightarrow K_{i-1}\left(O_{F}\right) \\
& \longrightarrow K_{i-1}(F) \longrightarrow \cdots \longrightarrow K_{1}\left(O_{F}\right) \longrightarrow K_{1}(F) \\
& \longrightarrow \bigoplus_{\mathfrak{m}} K_{0}\left(O_{F} / \mathfrak{m}\right) \longrightarrow K_{0}\left(O_{F}\right) \longrightarrow K_{0}(F)
\end{aligned}
$$

where $\mathfrak{m}$ runs over the set of all maximal ideals of $O_{F}$.

Moreover, C. Soulé could improve this result by showing that this long exact sequence breaks into short exact sequences for all positive integers $i$ (see [86, Théorème 1$]$ ):

$$
0 \longrightarrow K_{i}\left(O_{F}\right) \longrightarrow K_{i}(F) \longrightarrow \bigoplus_{\mathfrak{m}} K_{i-1}\left(O_{F} / \mathfrak{m}\right) \longrightarrow 0 .
$$

Since $O_{F} / \mathfrak{m}$ is a finite field, the vanishing of $K_{j}\left(O_{F} / \mathfrak{m}\right)$ whenever $j$ is even $\geq 2$ (see Corollary 5.9) then implies the following result.

Theorem 8.3. Let $F$ be any number field.

(a) For any odd integer $i \geq 3$, the inclusion $O_{F} \hookrightarrow F$ induces an isomorphism

$$
K_{i}\left(O_{F}\right) \stackrel{\cong}{\longrightarrow} K_{i}(F) .
$$


(b) For any even integer $i \geq 2$, there is a short exact sequence

$$
0 \longrightarrow K_{i}\left(O_{F}\right) \longrightarrow K_{i}(F) \longrightarrow \bigoplus_{\mathfrak{m}} K_{i-1}\left(O_{F} / \mathfrak{m}\right) \longrightarrow 0
$$

where $\mathfrak{m}$ runs over the set of all maximal ideals of $O_{F}$ and where $K_{i-1}\left(O_{F} / \mathfrak{m}\right)$ can be determined by Corollary 5.9.

Remark 8.4. Similar results hold for rings of $\mathcal{S}$-integers in $F$, where $\mathcal{S}$ is any set of places of $F$ (see [86, Théorème 1]).

The following finiteness result follows immediately from Theorems 8.1 and 8.3 .

Corollary 8.5. For any number field $F$ and any odd integer $i \geq 3$, the group $K_{i}(F)$ is finitely generated.

The next important information on the structure of the $K$-groups of number fields and rings of integers was obtained by A. Borel as a consequence of his study of the real cohomology of linear groups (see [31] or [32, Section 11]).

Theorem 8.6 (Borel). Let $F$ be a number field and let us write $[F: \mathbb{Q}]=r_{1}+2 r_{2}$, where $r_{1}$ is the number of distinct embeddings of $F$ into $\mathbb{R}$ and $r_{2}$ the number of distinct conjugate pairs of embeddings of $F$ into $\mathbb{C}$ with image not contained in $\mathbb{R}$.

(a) If $R$ denotes either the number field $F$ or its ring of algebraic integers $O_{F}$, then the rational cohomology of the special linear group $S L(R)$ is given by

$$
H^{*}(S L(R) ; \mathbb{Q}) \cong\left(\bigotimes_{1 \leq j \leq r_{1}} A_{j}\right) \otimes\left(\bigotimes_{1 \leq k \leq r_{2}} B_{k}\right)
$$

where $j$ runs over all distinct embeddings of $F$ into $\mathbb{R}, k$ over all distinct conjugate pairs of embeddings of $F$ into $\mathbb{C}$ with image not contained in $\mathbb{R}$, and where $A_{j}$ and $B_{k}$ are the following exterior algebras:

$$
\begin{aligned}
& A_{j}=\Lambda_{\mathbb{Q}}\left(x_{5}, x_{9}, x_{13}, \ldots, x_{4 l+1}, \ldots\right) \quad \text { and } \\
& B_{k}=\Lambda_{\mathbb{Q}}\left(x_{3}, x_{5}, x_{7}, \ldots, x_{2 l+1}, \ldots\right)
\end{aligned}
$$

with $\operatorname{deg}\left(x_{j}\right)=j$. 
(b) If $R$ denotes either the number field $F$ or its ring of algebraic integers $O_{F}$, then for any integer $i \geq 2$,

$$
K_{i}(R) \otimes \mathbb{Q} \cong \begin{cases}0, & \text { if } i \text { is even, } \\ \mathbb{Q}^{r_{1}+r_{2}}, & \text { if } i \equiv 1 \bmod 4, \\ \mathbb{Q}^{r_{2}}, & \text { if } i \equiv 3 \bmod 4 .\end{cases}
$$

As a consequence, we observe:

Corollary 8.7. If $R$ denotes a number field $F$ or its ring of integers $O_{F}$, then $K_{i}(R)$ is a torsion group for all even integers $i \geq 2$.

In order to summarize Theorem 8.1, Corollaries 8.5 and 8.7, we can formulate the following statement.

Corollary 8.8. Let $F$ be any number field, $O_{F}$ its ring of integers and $i$ a positive integer.

(a) $K_{i}(F)$ is finitely generated if $i$ is odd and $K_{i}(F)$ is a torsion group if $i$ is even.

(b) $K_{i}(F) /$ torsion is a free abelian group of finite rank (which is known by Theorem $8.6(b))$ for all positive integers $i$.

(c) $K_{i}\left(O_{F}\right)$ is finitely generated if $i$ is odd and $K_{i}\left(O_{F}\right)$ is finite if $i$ is even.

However, the structure of the groups $K_{i}(F)$ is quite complicated. In order to illustrate this, let us consider the subgroup $D_{i}(F)$ of $K_{i}(F)$ consisisting of all (infinitely) divisible elements in $K_{i}(F)$ (notice that $D_{i}(F)$ is not necessarily a divisible subgroup of $\left.K_{i}(F)\right)$ and prove the following surprising assertion.

Theorem 8.9. For any number field $F, D_{i}(F)=0$ if $i$ is an odd integer $\geq 1$ and $D_{i}(F)$ is a finite abelian group if $i$ is an even integer $\geq 2$.

Proof: Since $K_{i}(F)$ is a finitely generated abelian group when $i$ is odd according to Corollary 8.5 , it does not contain any non-trivial divisible element and $D_{i}(F)=0$. When $i$ is even, consider again the localization exact sequence

$$
0 \longrightarrow K_{i}\left(O_{F}\right) \longrightarrow K_{i}(F) \longrightarrow \bigoplus_{\mathfrak{m}} K_{i-1}\left(O_{F} / \mathfrak{m}\right) \longrightarrow 0 .
$$

By Corollary $5.9, K_{i-1}\left(O_{F} / \mathfrak{m}\right)$ is a finite cyclic group and contains therefore no non-trivial divisible elements. Consequently, the same is true for the direct sum $\bigoplus_{\mathfrak{m}} K_{i-1}\left(O_{F} / \mathfrak{m}\right)$. It then follows that all divisible elements in $K_{i}(F)$ actually belong to the image of the homomorphism $K_{i}\left(O_{F}\right) \rightarrow K_{i}(F)$ induced by the inclusion $O_{F} \hookrightarrow F$. Finally, the 
fact that $K_{i}\left(O_{F}\right)$ is finite by Corollary 8.8 (c) shows that $D_{i}(F)$ is finite. (The elements of $D_{i}(F)$ can be viewed as elements of $K_{i}\left(O_{F}\right)$, even if they are divisible only in $K_{i}(F)$.)

Remark 8.10. The situation is indeed quite strange because the group $D_{i}(F)$ does not vanish in general. This was proved in a very precise way by G. Banaszak in [23, Section VIII], and [24, Section II]. For any odd prime $p$, let us write $D_{i}(F)_{p}$ for the subgroup of $p$-torsion divisible elements in $K_{i}(F)$ (in other words, $D_{i}(F)_{p}$ is the $p$-component of $D_{i}(F)$ ). If $F$ is a totally real number field, $i=2 m$ an even integer with $m$ odd, G. Banaszak, together with M. Kolster, determined the order of the subgroup $D_{2 m}(F)_{p}$ (see [24, Theorem 3]): the order of $D_{2 m}(F)_{p}$ is exactly the $p$-adic absolute value of

$$
\frac{w_{m+1}(F) \zeta_{F}(-m)}{\prod_{v \mid p} w_{m}\left(F_{v}\right)}
$$

where $\zeta_{F}(-)$ is the Dedekind zeta function of $F, w_{m}(k)$ the biggest integer $s$ such that the exponent of the Galois group $\operatorname{Gal}\left(k\left(\xi_{s}\right) / k\right)$ divides $m$ for any field $k$ (here $\xi_{s}$ is an $s$-th primitive root of unity), and $F_{v}$ the completion of $F$ at $v$. For the case where $F$ is the field of rationals $\mathbb{Q}$, look at Remark 9.15 for a more explicit description of the order of the groups $D_{2 m}(\mathbb{Q})_{p}$.

Notice that the knowledge of $D_{2 m}(F)$ is of particular interest since it is related to the Lichtenbaum-Quillen conjecture in algebraic $K$-theory (see Remark 9.16 and [24, Section II.2]) and to étale $K$-theory (see [25, Section 3]).

In order to have an almost complete picture of the complexity of the structure of the algebraic $K$-groups of number fields, let us try to get analogous results for integral homology. Of course, the Hurewicz theorem modulo the Serre class of finitely generated abelian groups (see [82, Sections III.1 and III.2]) for the space $B S L\left(O_{F}\right)^{+}$enables us to deduce from Theorem 8.1 the following structure theorem for the integral homology of the special linear group over a ring of integers.

Corollary 8.11. For any number field $F$ and any integer $i \geq 0$, $H_{i}\left(S L\left(O_{F}\right) ; \mathbb{Z}\right)$ is a finitely generated abelian group.

The situation is more complicated for the special linear group $S L(F)$ over the number field $F$ itself: in fact, the structure of the groups $H_{i}(S L(F) ; \mathbb{Z})$ turns out to be similar to the structure of $K_{i}(F)$ described in Corollary $8.8(\mathrm{~b})$. 
Theorem 8.12. For any number field $F$ and any integer $i \geq 0$, the group $H_{i}(S L(F) ; \mathbb{Z})$ is the direct sum of a torsion group and a free abelian group of finite rank (which can be calculated by Theorem 8.6 (a)).

Proof: (See also [5, Section 2].) The proof is based on the results on the Postnikov invariants described in Section 7 . Let $\mathcal{C}$ denote the Serre class of all abelian torsion groups. Because $B S L(F)^{+}$is a simply connected infinite loop space, Corollary 7.16 implies that all Postnikov $k$-invariants of $B S L(F)^{+}$are cohomology classes of finite order. Therefore, Proposition 7.10 (b) provides a map

$$
f: B S L(F)^{+} \longrightarrow \prod_{j=2}^{\infty} K\left(K_{j}(F), j\right)
$$

which induces multiplication by the (finite) order of the corresponding $k$-invariant $k^{i+1}\left(B S L(F)^{+}\right)$on each homotopy group $\pi_{i}\left(B S L(F)^{+}\right) \cong$ $K_{i}(F), i \geq 2$. In particular, $f$ induces a $\mathcal{C}$-isomorphism on each homotopy group. Now, let us compose $f$ with the natural map

$$
\prod_{j=2}^{\infty} K\left(K_{j}(F), j\right) \longrightarrow \prod_{j=2}^{\infty} K\left(K_{j}(F) / \text { torsion, } j\right)
$$

which induces the quotient map (and thus a $\mathcal{C}$-isomorphism) on each homotopy group. If we denote by $Y$ this later space, this composition is a map

$$
\psi: B S L(F)^{+} \longrightarrow Y=\prod_{j=2}^{\infty} K\left(K_{j}(F) / \text { torsion, } j\right)
$$

inducing a $\mathcal{C}$-isomorphism on all homotopy groups and therefore also a $\mathcal{C}$-isomorphism

$$
\psi_{*}: H_{i}\left(B S L(F)^{+} ; \mathbb{Z}\right) \rightarrow H_{i}(Y ; \mathbb{Z})
$$

on all integral homology groups because of the $\bmod \mathcal{C}$ Whitehead theorem (see [82, Section III.4]). On the other hand, since $\pi_{i}(Y) \cong$ $K_{i}(F)$ torsion is finitely generated for all integers $i \geq 1$ by Corollary 8.8 (b), the homology groups $H_{i}(Y ; \mathbb{Z})$ of $Y$ are also finitely generated. Consequently, the group $H_{i}\left(B S L(F)^{+} ; \mathbb{Z}\right) / \operatorname{ker} \psi_{*} \cong$ image $\psi_{*}$ is also finitely generated and ker $\psi_{*}$ belongs to $\mathcal{C}$. If $T_{i}$ is written for the torsion subgroup of $H_{i}\left(B S L(F)^{+} ; \mathbb{Z}\right)$, it follows that $H_{i}\left(B S L(F)^{+} ; \mathbb{Z}\right) / T_{i}$ is finitely generated, i.e., free abelian of finite rank, since it is a quotient of $H_{i}\left(B S L(F)^{+} ; \mathbb{Z}\right) / \operatorname{ker} \psi_{*}$. Finally, this implies the vanishing of $\operatorname{Ext}\left(H_{i}\left(B S L(F)^{+} ; \mathbb{Z}\right) / T_{i}, T_{i}\right)$ and the splitting of the extension

$$
0 \longrightarrow T_{i} \longrightarrow H_{i}\left(B S L(F)^{+} ; \mathbb{Z}\right) \longrightarrow H_{i}\left(B S L(F)^{+} ; \mathbb{Z}\right) / T_{i} \longrightarrow 0 \text {. }
$$


The assertion then follows from the isomorphism

$$
H_{i}(S L(F) ; \mathbb{Z}) \cong H_{i}\left(B S L(F)^{+} ; \mathbb{Z}\right) .
$$

Because of Theorem 8.3 (b), the groups $K_{i}(F)$ are in general not finitely generated. By Serre class theory (see [82, Chapitre I]), this implies that the homology groups $H_{i}(S L(F) ; \mathbb{Z})$ are in general not finitely generated. However, this only happens because of their torsion subgroups. The next step would be to investigate the structure of the torsion subgroups of the groups $H_{i}(S L(F) ; \mathbb{Z})$. In particular, let us look at the subgroup $\bar{D}_{i}(F)$ of divisible elements in $H_{i}(S L(F) ; \mathbb{Z})$. Here again, an argument similar to the proof of Theorem 8.12 shows that this subgroup is relatively small in the following sense.

Theorem 8.13. For any number field $F$ and any integer $i \geq 0$, the abelian group $\bar{D}_{i}(F)$ is of finite exponent.

Proof: See [17, Theorem 1.1].

Remark 8.14. Observe that for any number field $F$, the homomorphism $K_{i}\left(O_{F}\right) \rightarrow K_{i}(F)$ induced by the inclusion $O_{F} \hookrightarrow F$ is always injective according to Theorem 8.3. The analogous assertion for the induced homomorphism $H_{i}\left(S L\left(O_{F}\right) ; \mathbb{Z}\right) \rightarrow H_{i}(S L(F) ; \mathbb{Z})$ is not true (see Remark 2.7 of [18]). However, one can prove (see Theorem 1.4 of [18]) the injectivity of the induced homomorphism $H_{i}\left(S L\left(O_{F}\right) ; \mathbb{Z}_{(p)}\right) \rightarrow$ $H_{i}\left(S L(F) ; \mathbb{Z}_{(p)}\right)$ in small dimensions, more precisely for $2 \leq i \leq$ $\min \left(2 p-2, d_{p}(F)+1\right)$, where $d_{p}(F)$ denotes the smallest positive integer $j$ for which $K_{j}(F)$ contains non-trivial $p$-torsion divisible elements $\left(d_{p}(F)\right.$ is an even integer according to Theorem 8.9 and we say that $d_{p}(F)=\infty$ if there are no $p$-torsion divisible elements in $K_{j}(F)$ for all $j \geq 1$ ).

\section{The algebraic $K$-theory of the ring of integers $\mathbb{Z}$}

If we apply the results of the previous sections to the special case of the ring of integers $\mathbb{Z}$, we first know that $E(\mathbb{Z})=S L(\mathbb{Z})$ by Theorem 2.10 and we may deduce from Section 8 the following result on the structure of the abelian groups $K_{i}(\mathbb{Z})$.

Theorem 9.1. For any positive integer $i$,

$$
K_{i}(\mathbb{Z})= \begin{cases}\mathbb{Z} \oplus \text { finite group, } & \text { if } i \equiv 1 \quad \bmod 4, i \geq 5, \\ \text { finite group, } & \text { otherwise } .\end{cases}
$$


Proof: Theorem 8.1 asserts that the groups $K_{i}(\mathbb{Z})$ are finitely generated abelian groups for all $i \geq 0$. Moreover, the rank of the free abelian group $K_{i}(\mathbb{Z}) /$ torsion is given by Theorem $8.6(\mathrm{~b})$, with $r_{1}=1$ and $r_{2}=0$.

In low dimensions, the $K$-groups of $\mathbb{Z}$ have been computed for $i \leq 4$ and partially determined for $i=5$.

Theorem 9.2. $K_{0}(\mathbb{Z}) \cong \mathbb{Z}, K_{1}(\mathbb{Z}) \cong \mathbb{Z} / 2, K_{2}(\mathbb{Z}) \cong \mathbb{Z} / 2, K_{3}(\mathbb{Z}) \cong$ $\mathbb{Z} / 48, K_{4}(\mathbb{Z})=0$ and $K_{5}(\mathbb{Z}) \cong \mathbb{Z} \oplus$ (3-torsion finite group).

Proof: See Theorem 1.2, Theorem 2.10 and Example 2.29 for the calculation of $K_{0}(\mathbb{Z}), K_{1}(\mathbb{Z})$ and $K_{2}(\mathbb{Z}),[\mathbf{5 5}]$ for the determination of $K_{3}(\mathbb{Z})$, [76], [88], [77], [99] and [95] for the vanishing of $K_{4}(\mathbb{Z})$, and [56] and [84] for the description of $K_{5}(\mathbb{Z})$.

Remark 9.3. The cyclic groups of order 2 in $K_{i}(\mathbb{Z})$ for $i=1$ and $i=$ 2 occur actually in all dimensions $i \equiv 1$ or $2 \bmod 8$, as observed by D. Quillen in [75].

Let us also look at the unstable Hurewicz homomorphisms

$$
\begin{aligned}
& h_{i}: K_{i}(\mathbb{Z}) \cong \pi_{i}\left(B S L(\mathbb{Z})^{+}\right) \\
& \quad \longrightarrow H_{i}\left(B S L(\mathbb{Z})^{+} ; \mathbb{Z}\right) \cong H_{i}(S L(\mathbb{Z}) ; \mathbb{Z}) \quad \text { for } i \geq 2
\end{aligned}
$$

and

$$
\begin{aligned}
& h_{i}: K_{i}(\mathbb{Z}) \cong \pi_{i}\left(B S t(\mathbb{Z})^{+}\right) \\
& \quad \longrightarrow H_{i}\left(B S t(\mathbb{Z})^{+} ; \mathbb{Z}\right) \cong H_{i}(\operatorname{St}(\mathbb{Z}) ; \mathbb{Z}) \quad \text { for } i \geq 3 .
\end{aligned}
$$

Theorem 9.4. The following sequences are exact:

(a) $\cdots \longrightarrow K_{4}(\mathbb{Z}) \stackrel{h_{4}}{\longrightarrow} H_{4}(S L(\mathbb{Z}) ; \mathbb{Z}) \longrightarrow \mathbb{Z} / 4 \longrightarrow K_{3}(\mathbb{Z}) \stackrel{h_{3}}{\longrightarrow}$ $H_{3}(S L(\mathbb{Z}) ; \mathbb{Z}) \longrightarrow 0$, where $\operatorname{ker} h_{3} \cong K_{2}(\mathbb{Z}) \star K_{1}(\mathbb{Z}) \cong \mathbb{Z} / 2$,

(b) $\cdots \longrightarrow K_{5}(\mathbb{Z}) \stackrel{h_{5}}{\longrightarrow} H_{5}(S t(\mathbb{Z}) ; \mathbb{Z}) \longrightarrow \underbrace{K_{3}(\mathbb{Z}) \otimes K_{1}(\mathbb{Z})}_{\cong \mathbb{Z} / 2} \stackrel{\star}{\longrightarrow}$

$$
K_{4}(\mathbb{Z}) \stackrel{h_{4}}{\longrightarrow} H_{4}(S t(\mathbb{Z}) ; \mathbb{Z}) \longrightarrow 0 .
$$

Proof: The unstable Whitehead exact sequence (see [102]) of the simply connected space $B S L(\mathbb{Z})^{+}$is

$$
\begin{aligned}
\cdots \longrightarrow K_{4}(\mathbb{Z}) \stackrel{h_{4}}{\longrightarrow} H_{4}\left(B S L(\mathbb{Z})^{+} ; \mathbb{Z}\right) \longrightarrow \Gamma_{3}\left(B S L(\mathbb{Z})^{+}\right) \\
\longrightarrow K_{3}(\mathbb{Z}) \stackrel{h_{3}}{\longrightarrow} H_{3}\left(B S L(\mathbb{Z})^{+} ; \mathbb{Z}\right) \longrightarrow 0
\end{aligned}
$$


and

$$
\Gamma_{3}\left(B S L(\mathbb{Z})^{+}\right) \cong \Gamma\left(\pi_{2}\left(B S L(\mathbb{Z})^{+}\right)\right) \cong \Gamma\left(K_{2}(\mathbb{Z})\right) \cong \Gamma(\mathbb{Z} / 2) \cong \mathbb{Z} / 4,
$$

where $\Gamma(-)$ is the quadratic functor defined on abelian groups by J. H. C. Whitehead in Section 5 of [102] (see also [4, Satz 1.5]). This gives the exact sequence (a) and Theorem 6.15 shows that the kernel of $h_{3}$ is exactly the image of the product $K_{2}(\mathbb{Z}) \otimes K_{1}(\mathbb{Z}) \stackrel{\star}{\longrightarrow} K_{3}(\mathbb{Z})$. Assertion (b) follows directly from Theorem 6.16 .

Of course, this also produces (co)homological results. According to Remark 7.9 (or Lemma 1.2 of [4]), we have the homotopy equivalence

$$
B G L(\mathbb{Z})^{+} \simeq B S L(\mathbb{Z})^{+} \times B \mathbb{Z} / 2
$$

and it is therefore sufficient to investigate the homology of the universal cover $B S L(\mathbb{Z})^{+}$of $B G L(\mathbb{Z})^{+}$. Let us first recall Theorem 8.6 (a) on the rational cohomology of $S L(\mathbb{Z})$.

Theorem 9.5. $H^{*}(S L(\mathbb{Z}) ; \mathbb{Q}) \cong \Lambda_{\mathbb{Q}}\left(x_{5}, x_{9}, x_{13}, \ldots, x_{4 l+1}, \ldots\right)$, where $\operatorname{deg}\left(x_{4 l+1}\right)=4 l+1$.

We may determine the homology of $S L(\mathbb{Z})$ and $S t(\mathbb{Z})$ in small dimensions from Theorems 9.2 and 9.3 (see also [7] for the relations between $H_{*}(S L(\mathbb{Z}) ; \mathbb{Z})$ and $\left.H_{*}(S t(\mathbb{Z}) ; \mathbb{Z})\right)$.

Theorem 9.6. $H_{2}(S L(\mathbb{Z}) ; \mathbb{Z}) \cong \mathbb{Z} / 2, H_{3}(S L(\mathbb{Z}) ; \mathbb{Z}) \cong \mathbb{Z} / 24$, $H_{4}(S L(\mathbb{Z}) ; \mathbb{Z}) \cong \mathbb{Z} / 2, H_{3}(S t(\mathbb{Z}) ; \mathbb{Z}) \cong \mathbb{Z} / 48, H_{4}(S t(\mathbb{Z}) ; \mathbb{Z})=0$, $H_{5}(S t(\mathbb{Z}) ; \mathbb{Z}) \cong \mathbb{Z} \oplus(3$-torsion finite group $)$ and there is a short exact sequence

$$
0 \longrightarrow K_{5}(\mathbb{Z}) \stackrel{h_{5}}{\longrightarrow} H_{5}(S t(\mathbb{Z}) ; \mathbb{Z}) \longrightarrow \mathbb{Z} / 2 \longrightarrow 0
$$

in which $h_{5}$ is an isomorphism on the torsion subgroup of $K_{5}(\mathbb{Z})$ and multiplication by 2 on the infinite cyclic summand of $K_{5}(\mathbb{Z})$.

Proof: Theorems 6.1 and 9.2 imply that $H_{2}(S L(\mathbb{Z}) ; \mathbb{Z}) \cong K_{2}(\mathbb{Z}) \cong \mathbb{Z} / 2$ and that $H_{3}(S t(\mathbb{Z}) ; \mathbb{Z}) \cong K_{3}(\mathbb{Z}) \cong \mathbb{Z} / 48$. It follows from the vanishing of $K_{4}(\mathbb{Z})$ and Theorem 9.4 that $H_{3}(S L(\mathbb{Z}) ; \mathbb{Z}) \cong \mathbb{Z} / 24$ (see also [4, Satz $1.5]), H_{4}(S L(\mathbb{Z}) ; \mathbb{Z}) \cong \mathbb{Z} / 2$ and $H_{4}(S t(\mathbb{Z}) ; \mathbb{Z})=0$. Finally, it is possible to show that the term $Q(\mathbb{Z})$ occuring in Theorem 6.16 is trivial (see $\left[\mathbf{1 3}\right.$, Theorem 3], or [14, Proposition 5.1]). Consequently, $h_{5}: K_{5}(\mathbb{Z}) \rightarrow$ $H_{5}(S t(\mathbb{Z}) ; \mathbb{Z})$ is injective because $K_{4}(\mathbb{Z})=0$ and we get the desired exact sequence. Finally, the effect of $h_{5}$ on the infinite cyclic summand of $K_{5}(\mathbb{Z})$ is explained by Theorem 1.5 of $[7]$. 
The first more general result on the torsion of the the algebraic $K$-groups of $\mathbb{Z}$ has been obtained by D. Quillen in 1976 (see [75]). Remember that he had already computed the $K$-theory of finite fields. By studying the map $K_{i}(\mathbb{Z}) \rightarrow K_{i}\left(\mathbb{F}_{p}\right)$ induced by the reduction $\bmod p: \mathbb{Z} \rightarrow$ $\mathbb{F}_{p}$ for various primes $p$, he could prove the following relationship between the order of the torsion subgroups of the $K$-groups of $\mathbb{Z}$ and the denominators of the Bernoulli numbers.

Definition 9.7. The Bernoulli numbers are the rational numbers $B_{m}$ occuring in the power series

$$
\frac{t}{e^{t}-1}=1+\sum_{m=1}^{\infty} \frac{B_{m}}{m !} t^{m}
$$

of the complex function $f(t)=\frac{t}{e^{t}-1}$. It is not hard to check that

$$
B_{1}=-\frac{1}{2} \quad \text { and } \quad B_{m}=0 \text { for } m \text { odd } \geq 3 .
$$

The first Bernoulli numbers are

$$
\begin{aligned}
& B_{2}=\frac{1}{6}, \quad B_{4}=-\frac{1}{30}, \quad B_{6}=\frac{1}{42}, \\
& B_{8}=-\frac{1}{30}, \quad B_{10}=\frac{5}{66}, \quad B_{12}=-\frac{691}{2730}, \\
& B_{14}=\frac{7}{6}, \quad B_{16}=-\frac{3617}{510}, \quad B_{18}=\frac{43867}{798} .
\end{aligned}
$$

Definition 9.8. For any positive even integer $m$, let

$$
E_{m}=\text { denominator }\left(\frac{B_{m}}{m}\right) \text {. }
$$

For instance,

$$
\begin{aligned}
& E_{2}=12, \quad E_{4}=120, \quad E_{6}=252, \\
& E_{8}=240, \quad E_{10}=132, \quad E_{12}=32760, \\
& E_{14}=12, \quad E_{16}=8160, \quad E_{18}=14364 \text {. }
\end{aligned}
$$

It turns out that the numbers $E_{m}$ are completely determined by the following property observed by K. von Staudt in 1845 .

Lemma 9.9. Let $p$ be a prime and $m$ a positive even integer. Then, for $s \geq 1, p^{s}$ divides $E_{m}$ if and only if $(p-1) p^{(s-1)}$ divides $m$.

Proof: See $[\mathbf{3 4}$, p. 410, Satz 4], [98, p. 56, Theorem 5.10], or [66, Appendix B, Theorem B.4]. 
D. Quillen exhibited the following torsion classes in the algebraic $K$-theory of $\mathbb{Z}$.

Theorem 9.10 (Quillen). For any positive even integer $j$, the group $K_{4 j-1}(\mathbb{Z})$ contains a cyclic subgroup $Q_{4 j-1}$ of order $2 E_{2 j}$. If $j$ is even, $Q_{4 j-1}$ is a direct summand of $K_{4 j-1}(\mathbb{Z})$. If $j$ is odd, the odd-torsion part of $Q_{4 j-1}$ is a direct summand of $K_{4 j-1}(\mathbb{Z})$ and the 2-torsion part of $Q_{4 j-1}$ (which is $\cong \mathbb{Z} / 8$ ) is contained in a cyclic direct summand of order 16 of $K_{4 j-1}(\mathbb{Z})$.

Proof: See [75] for the detection of the subgroup $Q_{4 j-1}$ of order $2 E_{2 j}$ in $K_{4 j-1}(\mathbb{Z})$ and the discussion of the case where $j$ is even, and Theorem 4.8 of $[\mathbf{3 6}]$ for the case $j$ odd.

We then may conclude the next consequence from Lemma 9.9 and Theorem 9.10.

Corollary 9.11. The group $K_{i}(\mathbb{Z})$ contains a cyclic subgroup of order 16 if $i \equiv 3 \bmod 8$ and of order $2(i+1)_{2}$ if $i \equiv 7 \bmod 8$, where $(i+1)_{2}$ denotes the 2-primary part of the integer $(i+1)$.

Another very surprising result was proved by C. Soulé in 1979 when he explained that in fact the numerators of the Bernoulli numbers also play a role in the investigation of the torsion in the groups $K_{i}(\mathbb{Z})$. Recall the following definition.

Definition 9.12. A prime number $p$ is called irregular if there exists a positive even integer $m$ such that $p$ divides the numerator of $\frac{B_{m}}{m}$ (see [34, p. 393-414], or [98, p. 6 and Section 5.3], for more details). For instance, 691 is an irregular prime since $\frac{B_{12}}{12}=-\frac{691}{32760}$. It turns out that $p$ is irregular if and only if $p$ divides the class number of the cyclotomic field $\mathbb{Q}\left(\xi_{p}\right)$, where $\xi_{p}$ is a $p$-th primitive root of unity; moreover, an irregular prime $p$ is called properly irregular if $p$ does not divide the class number of the maximal real subfield of $\mathbb{Q}\left(\xi_{p}\right)$ (see $[\mathbf{9 8}$, p. 39 and p. 165]). A regular prime is a prime number which is not irregular. Notice that there are infinitely many irregular primes but that it is still not known whether there are finitely or infinitely many regular primes.

Theorem 9.13 (Soulé). If $p$ is a properly irregular prime number and if $m$ is a positive even integer $<p$ such that $p$ divides the numerator of $\frac{B_{m+1}}{m+1}$, then the algebraic $K$-theory group $K_{2 m}(\mathbb{Z})$ contains an element whose order is equal to the p-primary part of $\frac{B_{m+1}}{m+1}$. 
Proof: See [85, Section IV.3, Théorème 6], where the argument is based on the investigation of the relationships between algebraic $K$-theory and étale cohomology.

Example 9.14. The group $K_{22}(\mathbb{Z})$ contains 691-torsion.

Remark 9.15. Consider again the short exact sequences

$$
0 \longrightarrow K_{2 m}(\mathbb{Z}) \longrightarrow K_{2 m}(\mathbb{Q}) \longrightarrow \bigoplus_{p \text { prime }} K_{2 m-1}(\mathbb{Z} / p) \longrightarrow 0
$$

given by Theorem 8.3 for all positive integers $m$. The torsion elements of $K_{2 m}(\mathbb{Z})$ detected by C. Soulé and presented in Theorem 9.13 also belong to the group $K_{2 m}(\mathbb{Q})$ and play a special role in that group with respect to the subgroup $D_{2 m}(\mathbb{Q})$ of divisible elements in $K_{2 m}(\mathbb{Q})$ (see Theorem 8.9 and Remark 8.10). In fact G. Banaszak determined the precise order of the $p$-primary component $D_{2 m}(\mathbb{Q})_{p}$ of $D_{2 m}(\mathbb{Q})$ as follows (see $[\mathbf{2 4}$, Theorem 3]): if $m$ is an odd integer and $p$ an odd prime, then the order of $D_{2 m}(\mathbb{Q})_{p}$ is equal to the $p$-adic absolute value of the numerator of $\frac{B_{m+1}}{m+1}$. For example, $D_{22}(\mathbb{Q})$ is cyclic of order 691 .

Remark 9.16. The torsion in the groups $K_{i}(\mathbb{Z})$ is really mysterious and contains a lot of number theoretical information. It is the object of the following (still open) conjecture due to S. Lichtenbaum and D. Quillen: if $m$ is a positive even integer, then the quotient of the order of $K_{2 m-2}(\mathbb{Z})$ by the order of $K_{2 m-1}(\mathbb{Z})$ should be equal to the absolute value of $\frac{B_{m}}{m}$, up to a power of 2 (see [57], [85, Section I.1], or [58, p. 102-103]).

Thus, apart from some classes of order 2, the known torsion classes in the algebraic $K$-theory of $\mathbb{Z}$ which occur in odd degrees, respectively in even degrees, are related to the denominators, respectively to the numerators, of the Bernoulli numbers.

The next attempt to understand the $K$-theory of $\mathbb{Z}$ was made by M. Bökstedt in 1984 (see [30]): he tried to construct a model for the algebraic $K$-theory space $B G L(\mathbb{Z})^{+}$and proved that this model detects the known torsion classes at the prime 2 . His idea was simple and excellent: he considered the classifying space $B O$ of the orthogonal group, the classifying space $B U$ of the unitary group and, for a prime $p$, the $K$-theory space $B G L\left(\mathbb{F}_{p}\right)^{+}$. Then, he introduced a space $J(p)$ which is 
defined as the pull-back of the following diagram:

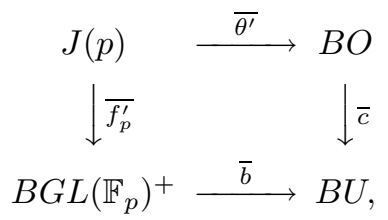

where $\bar{c}$ is the complexification and $\bar{b}$ the composition of the plus construction of the Brauer lift $B G L\left(\mathbb{F}_{p}\right) \rightarrow F \Psi^{p}$ with the inclusion $\varphi: F \Psi^{p} \hookrightarrow B U$ (see Definitions 5.1 and 5.7). Observe that the homotopy fiber of both horizontal maps in the above diagram is $\Omega B U \simeq U$. A direct calculation shows that $\pi_{1} J(p) \cong \mathbb{Z} \oplus \mathbb{Z} / 2$ and we know that $K_{1}(\mathbb{Z}) \cong \mathbb{Z} / 2$. Consequently, let us write $J K(\mathbb{Z}, p)$ for the covering space of $J(p)$ corresponding to the factor $\mathbb{Z} / 2$. It turns out that $J K(\mathbb{Z}, p)$ is the pull-back of the following diagram:

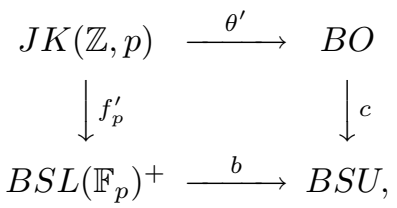

where the bottom arrow is the universal cover of the corresponding line in the previous diagram. In order to approximate the 2-torsion of the $K$-groups of $\mathbb{Z}$, M. Bökstedt chose a prime $p \equiv 3$ or $5 \bmod 8$, completed all spaces at 2 , and constructed a map $\psi:\left(B G L(\mathbb{Z})^{+}\right)_{2}^{\wedge} \rightarrow J K(\mathbb{Z}, p)_{2}^{\wedge}$ for which he was able to prove:

Theorem 9.17 (Bökstedt). The map $\Omega \psi: \Omega\left(B G L(\mathbb{Z})^{+}\right)_{2}^{\wedge} \rightarrow \Omega J K(\mathbb{Z}, p)_{2}^{\wedge}$ is a retraction. In particular, the map $\psi:\left(B G L(\mathbb{Z})^{+}\right)_{2}^{\wedge} \rightarrow J K(\mathbb{Z}, p)_{2}^{\wedge}$ induces a split surjection on all homotopy groups.

Proof: See [30, Theorem 2].

Observe at that point that it is easy to compute the homotopy groups of the space $J K(\mathbb{Z}, p)$ : consequently, Bökstedt's theorem provides actually a direct summand of each group $K_{i}(\mathbb{Z}) \otimes \mathbb{Z}_{2}^{\wedge}$, where $\mathbb{Z}_{2}^{\wedge}$ denotes the ring of 2-adic integers. Notice that $\psi$ induces a map

$$
\bar{\psi}:\left(B G L(\mathbb{Z})^{+} \times S^{1}\right)_{2}^{\wedge} \rightarrow J(p)_{2}^{\wedge}
$$

and that the localization exact sequence (see Theorem 8.3 and Remark 8.4) gives the following short exact sequence for all integers $i \geq 1$ :

$$
0 \longrightarrow K_{i}(\mathbb{Z}) \longrightarrow K_{i}\left(\mathbb{Z}\left[\frac{1}{2}\right]\right) \longrightarrow K_{i-1}\left(\mathbb{F}_{2}\right) \longrightarrow 0 \text {. }
$$


However, $K_{i-1}\left(\mathbb{F}_{2}\right) \otimes \mathbb{Z}_{2}^{\wedge}$ is always trivial according to Corollary 5.9 except if $i=1$, where $K_{0}\left(\mathbb{F}_{2}\right) \otimes \mathbb{Z}_{2}^{\wedge} \cong \mathbb{Z}_{2}^{\wedge}$. Therefore, $\left(B G L(\mathbb{Z})^{+} \times S^{1}\right)_{2}^{\wedge} \cong$ $\left(B G L\left(\mathbb{Z}\left[\frac{1}{2}\right]\right)^{+}\right)_{2}^{\wedge}$ and $\bar{\psi}$ is actually a map $\left(B G L\left(\mathbb{Z}\left[\frac{1}{2}\right]\right)^{+}\right)_{2}^{\wedge} \rightarrow J(p)_{2}^{\wedge}$ which induces also a split surjection on all homotopy groups.

A very significant step was made by V. Voevodsky in 1997, when he proved the Milnor conjecture [95] which asserts that if $F$ is a field of characteristic $\neq 2$, then $K_{i}^{M}(F) / 2 K_{i}^{M}(F) \cong H_{\text {êt }}^{i}(F ; \mathbb{Z} / 2)$ (see Definition 2.33). This fundamental theorem has many deep consequences. In particular, J. Rognes and C. Weibel were then able to use it in order to calculate the $E^{2}$-term of the Bloch-Lichtenbaum spectral sequence $E_{2}^{s, t} \Longrightarrow K_{-s-t}(\mathbb{Q} ; \mathbb{Z} / 2)$ and, after a very tricky study of its differentials, to determine the groups $K_{i}(\mathbb{Q} ; \mathbb{Z} / 2)$. They could then deduce from the localization exact sequence the calculation of $K_{i}(\mathbb{Z} ; \mathbb{Z} / 2)$ for all integers $i$. At that point, they were very lucky since all elements of the groups $K_{i}(\mathbb{Z} ; \mathbb{Z} / 2)$ were detected by the elements of $K_{i}(\mathbb{Z})$ which were already known by Theorem 9.2, Remark 9.3, Theorem 9.10 and Corollary 9.11. Consequently, they obtained the following complete calculation of the 2-torsion of the groups $K_{i}(\mathbb{Z})$.

Theorem 9.18 (Voevodsky, Rognes-Weibel). $K_{1}(\mathbb{Z}) \cong \mathbb{Z} / 2$ and for $i \geq 2$,

$K_{i}(\mathbb{Z}) \cong \begin{cases}\mathbb{Z} \oplus \mathbb{Z} / 2 \oplus \text { finite odd torsion group, } & \text { if } i \equiv 1 \bmod 8, \\ \mathbb{Z} / 2 \oplus \text { finite odd torsion group, } & \text { if } i \equiv 2 \bmod 8, \\ \mathbb{Z} / 16 \oplus \text { finite odd torsion group, } & \text { if } i \equiv 3 \bmod 8, \\ \mathbb{Z} \oplus \text { finite odd torsion group, } & \text { if } i \equiv 5 \bmod 8, \\ \mathbb{Z} /\left(2(i+1)_{2}\right) \oplus \text { finite odd torsion group, } & \text { if } i \equiv 7 \bmod 8, \\ \text { finite odd torsion group, } & \text { otherwise. }\end{cases}$

Proof: See [99, Table 1], and [77, Theorem 0.6].

Remark 9.19. W. Browder had already observed in [36, Theorem 4.8], that the cyclic factor of order 16 comes periodically in all groups $K_{i}(\mathbb{Z})$ with $i \equiv 3 \bmod 8$.

This theorem has an immediate crucial topological consequence. Consider any prime $p \equiv 3$ or $5 \bmod 8$ and the above map

$$
\psi:\left(B G L(\mathbb{Z})^{+}\right)_{2}^{\wedge} \rightarrow J K(\mathbb{Z}, p)_{2}^{\wedge}
$$

which induces a split surjection on all homotopy groups. It is easy to check that Theorem 9.18 shows that the homotopy groups of $\left(B G L(\mathbb{Z})^{+}\right)_{2}^{\wedge}$ and of $J K(\mathbb{Z}, p)_{2}^{\wedge}$ are the same. Therefore, Theorem 9.17 
implies that the induced homomorphism

$$
\psi_{*}: \pi_{i}\left(\left(B G L(\mathbb{Z})^{+}\right)_{2}^{\wedge}\right) \rightarrow \pi_{i}\left(J K(\mathbb{Z}, p)_{2}^{\wedge}\right)
$$

is an isomorphism for all positive integers $i$. This and a similar argument for the map

$$
\bar{\psi}:\left(B G L\left(\mathbb{Z}\left[\frac{1}{2}\right]\right)^{+}\right)_{2}^{\wedge} \rightarrow J(p)_{2}^{\wedge}
$$

imply the following result.

Corollary 9.20. There are homotopy equivalences

$$
\left(B G L(\mathbb{Z})^{+}\right)_{2}^{\wedge} \simeq J K(\mathbb{Z}, p)_{2}^{\wedge}
$$

and

$$
\left(B G L\left(\mathbb{Z}\left[\frac{1}{2}\right]\right)^{+}\right)_{2}^{\wedge} \simeq J(p)_{2}^{\wedge}
$$

Consequently, we may deduce the following theorem.

Theorem 9.21. For any prime $p \equiv 3$ or $5 \bmod 8$, one has the following commutative diagrams in which the rows are fibrations and where the right square is a pull-back square:

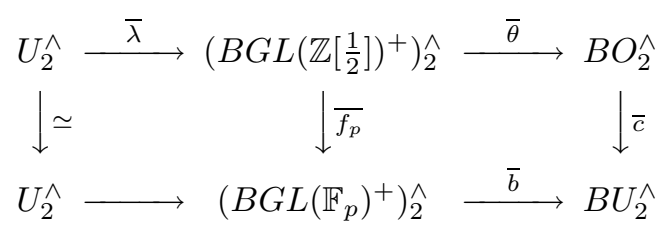

and

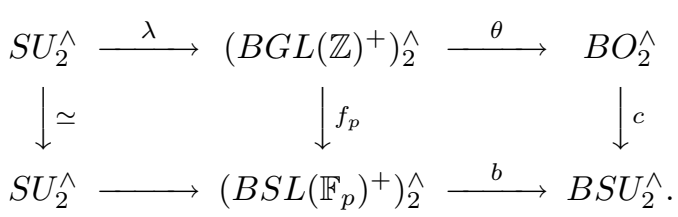

Here, the maps $\bar{\theta}, \overline{f_{p}}, \theta$ and $f_{p}$ are the compositions of the homotopy equi-

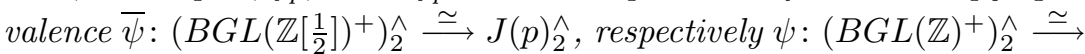
$J K(\mathbb{Z}, p)_{2}^{\wedge}$, with the maps $\bar{\theta}^{\prime}, \overline{f_{p}^{\prime}}, \theta^{\prime}$ and $f_{p}^{\prime}$. Moreover, $\bar{\theta}$ and $\theta$ are the maps induced by the inclusions $\mathbb{Z}\left[\frac{1}{2}\right] \hookrightarrow \mathbb{R}$ and $\mathbb{Z} \hookrightarrow \mathbb{R}$, and $\overline{f_{p}}$ and $f_{p}$ are induced by the reduction $\bmod p$.

Proof: This follows from the two diagrams introduced above and from Corollary 9.20. A careful study of Bökstedt's construction implies the identification of the maps $\bar{\theta}, \theta, \overline{f_{p}}, f_{p}$. 
This result provides a very complete knowledge of the homotopy type of the $K$-theory space $B G L(\mathbb{Z})^{+}$at the prime 2 . First of all, the homotopy groups $K_{i}(\mathbb{Z})=\pi_{i}\left(B G L(\mathbb{Z})^{+}\right)$of this space are known (after 2 -completion) by Theorem 9.18 . Then, it is also possible to determine at the prime 2 the Hurewicz homomorphism $h_{i}: K_{i}(\mathbb{Z}) \rightarrow H_{i}(G L(\mathbb{Z}) ; \mathbb{Z})$. If $i \equiv 1 \bmod 4$ and $i \geq 5$, then $K_{i}(\mathbb{Z}) \cong \mathbb{Z} \oplus$ (finite group) by Theorem 9.1. Let us call $b_{i}$ a generator of the infinite cyclic summand of $K_{i}(\mathbb{Z})$. On the other hand, it follows from Theorem 9.5 that $H_{*}(G L(\mathbb{Z}) ; \mathbb{Q}) \cong$ $\Lambda_{\mathbb{Q}}\left(x_{5}, x_{9}, x_{13}, \ldots, x_{4 l+1}, \ldots\right)$, where the elements $x_{4 l+1}$ are primitive generators of degree $4 l+1$. Thus, for any integer $i \equiv 1 \bmod 4($ with $i \geq 5)$ there exists a generator $a_{i}$ of an infinite cyclic summand of $H_{i}(G L(\mathbb{Z}) ; \mathbb{Z})$ with the property that

$$
h_{i}\left(b_{i}\right)= \pm \mu_{i} a_{i}+(\text { torsion element }),
$$

where $\mu_{i}$ is a positive integer. By using the fact that the Hurewicz homomorphism $\pi_{i}(S U) \rightarrow H_{i}(S U ; \mathbb{Z})$ acts in some sense as multiplication by $\pm\left(\frac{i-1}{2}\right)$ ! (see [40, Théorème 6]) and the map $\lambda: S U_{2}^{\wedge} \rightarrow\left(B G L(\mathbb{Z})^{+}\right)_{2}^{\wedge}$ provided by Theorem 9.21, it is not difficult to compute the 2-primary part $\left(\mu_{i}\right)_{2}$ of these integers $\mu_{i}$.

Corollary 9.22. For all integers $i \equiv 1 \bmod 4$ with $i \geq 5,\left(\mu_{i}\right)_{2}=$ $\left(\left(\frac{i-1}{2}\right) !\right)_{2}$.

Proof: See [21, Théorème 4.17].

Remark 9.23. In a similar way, one can compute the effect of the Hurewicz homomorphism on the 2-torsion classes of $K_{*}(\mathbb{Z})$ (see $[\mathbf{2 1}$, Théorème 4.22]).

In order to understand the homotopy type of $B G L(\mathbb{Z})^{+}$, one also needs to know its Postnikov $k$-invariants

$$
k^{i+1}\left(B G L(\mathbb{Z})^{+}\right) \in H^{i+1}\left(B G L(\mathbb{Z})^{+}[i-1] ; K_{i}(\mathbb{Z})\right)
$$

(see Section 7). We know from Theorem 7.17 that all $k$-invariants $k^{i+1}\left(B G L(\mathbb{Z})^{+}\right)$are cohomology classes of finite order.

Definition 9.24. For any $i \geq 2$, let $\rho_{i}$ denote the order of the $k$-invariant $k^{i+1}\left(B G L(\mathbb{Z})^{+}\right)$in $H^{i+1}\left(B G L(\mathbb{Z})^{+}[i-1] ; K_{i}(\mathbb{Z})\right)$. 
Corollary 9.25. For all integers $i \geq 2$, the 2-primary part $\left(\rho_{i}\right)_{2}$ of the integer $\rho_{i}$ is given by

$$
\left(\rho_{i}\right)_{2}= \begin{cases}\left(\left(\frac{i-1}{2}\right) !\right)_{2}, & \text { if } i \equiv 1 \bmod 4, \\ 2, & \text { if } i \equiv 2 \bmod 8 \text { and } i \geq 10, \text { or if } i=3 \text { or } 7, \\ 16, & \text { if } i \equiv 3 \bmod 8 \text { and } i \geq 11, \text { or if } i=15, \\ 2(i+1)_{2}, & \text { if } i \equiv 7 \bmod 8 \text { and } i \geq 23, \\ 1, & \text { otherwise. }\end{cases}
$$

Proof: This follows directly from Proposition 7.10 and the description of the Hurewicz homomorphism at the prime 2 provided by Corollary 9.22 and Remark 9.23 (see [21, Théorème 5.15] for more details).

The fibration

$$
S U_{2}^{\wedge} \stackrel{\lambda}{\longrightarrow}\left(B G L(\mathbb{Z})^{+}\right)_{2}^{\wedge} \stackrel{\theta}{\longrightarrow} B O_{2}^{\wedge}
$$

given by Theorem 9.21 enables us to deduce two other important properties of the algebraic $K$-theory space of $\mathbb{Z}$. Let us first completely compute the 2 -adic product structure of $K_{*}(\mathbb{Z})$.

Definition 9.26. The 2-adic product map in $K_{*}(\mathbb{Z})$ is the composition

$$
\star: K_{i}(\mathbb{Z}) \otimes K_{j}(\mathbb{Z}) \longrightarrow K_{i+j}(\mathbb{Z}) \longrightarrow K_{i+j}(\mathbb{Z}) \otimes \mathbb{Z}_{2}^{\wedge},
$$

where the first arrow is the usual $K$-theoretical product defined in Definition 4.5 and the second the tensor product of $K_{i+j}(\mathbb{Z})$ with the inclusion of $\mathbb{Z}$ into the ring of 2 -adic integers $\mathbb{Z}_{2}^{\wedge}(i, j \geq 1)$. We continue to denote this product by the symbol $\star$.

Theorem 9.27. The 2-adic product

$$
\star: K_{i}(\mathbb{Z}) \otimes K_{j}(\mathbb{Z}) \longrightarrow K_{i+j}(\mathbb{Z}) \otimes \mathbb{Z}_{2}^{\wedge}
$$

is trivial for all positive integers $i$ and $j$, except if $i \equiv j \equiv 1 \bmod 8$ or $i \equiv 1 \bmod 8$ and $j \equiv 2 \bmod 8($ or $i \equiv 2 \bmod 8$ and $j \equiv 1 \bmod 8)$, where its image is cyclic of order 2. In both exceptional cases the non-trivial element in the image of the 2-adic product map is the product of two elements of order 2 . 
Proof: The 2-adic product $\star: K_{i}(\mathbb{Z}) \otimes K_{j}(\mathbb{Z}) \longrightarrow K_{i+j}(\mathbb{Z}) \otimes \mathbb{Z}_{2}^{\wedge}$ is clearly trivial for dimension reasons (see Theorem 9.18) whenever $i$ and $j$ do not belong to one of the following six cases:

$$
\begin{array}{lllll}
i \equiv 1 & \bmod 8 & \text { and } & j \equiv 1 & \bmod 8, \\
i \equiv 1 & \bmod 8 & \text { and } & j \equiv 2 & \bmod 8, \\
i \equiv 2 & \bmod 8 & \text { and } & j \equiv 5 & \bmod 8 \\
i \equiv 2 & \bmod 8 & \text { and } & j \equiv 7 & \bmod 8 \\
i \equiv 3 & \bmod 8 & \text { and } & j \equiv 7 & \bmod 8 \\
i \equiv 5 & \bmod 8 & \text { and } & j \equiv 5 & \bmod 8 .
\end{array}
$$

In order to compute these six products, let us consider the following commutative diagram induced by the inclusion $\mathbb{Z} \hookrightarrow \mathbb{R}$ :

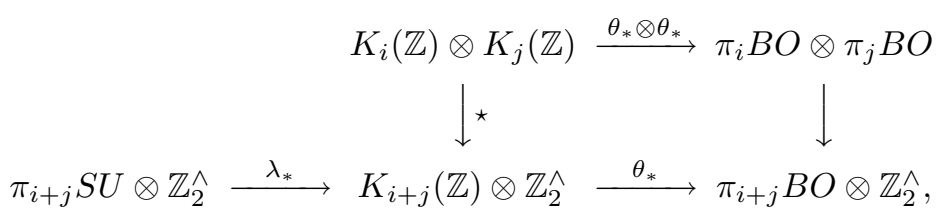

where the right vertical arrow is the composition of the product map in $\pi_{*} B O$ with the tensor product with $\mathbb{Z}_{2}^{\wedge}$ and where the bottom sequence is the homotopy exact sequence of the top fibration of the second diagram in Theorem 9.21. If $i+j$ is even, $\theta_{*}$ is injective since $\pi_{i+j} S U=0$. Consequently, this diagram detects the 2 -adic product $K_{i}(\mathbb{Z}) \star K_{j}(\mathbb{Z})$ when $i+j$ is even. This produces the calculation of the product in three of the above six cases. The 2-adic product turns out to be trivial in the last two cases. For the case $i \equiv j \equiv 1 \bmod 8$, recall that $K_{i}(\mathbb{Z}) \cong \mathbb{Z} \oplus \mathbb{Z} / 2 \oplus$ (finite odd torsion group) if $i \equiv 1 \bmod 8($ and $i \geq 9)$ and that $K_{i}(\mathbb{Z}) \cong$ $\mathbb{Z} / 2 \oplus$ (finite odd torsion group) if $i \equiv 2 \bmod 8$ (see Theorem 9.18). Let us denote by $y_{i}$, respectively by $z_{i}$, the element of order 2 in $K_{i}(\mathbb{Z})$ when $i \equiv 1 \bmod 8$, respectively when $i \equiv 2 \bmod 8$. Our argument shows that if $i \equiv j \equiv 1 \bmod 8$, the 2 -adic product $\star: K_{i}(\mathbb{Z}) \otimes K_{j}(\mathbb{Z}) \rightarrow K_{i+j}(\mathbb{Z}) \otimes \mathbb{Z}_{2}^{\wedge}$ satisfies

$$
y_{i} \star y_{j}=z_{i+j}
$$

(where $z_{i+j}$ is also written for the image of the element $z_{i+j}$ of $K_{i+j}(\mathbb{Z})$ under the homomorphism $\left.K_{i+j}(\mathbb{Z}) \rightarrow K_{i+j}(\mathbb{Z}) \otimes \mathbb{Z}_{2}^{\wedge}\right)$ and vanishes on other elements. In particular, we get the isomorphism

$$
K_{i}(\mathbb{Z}) \otimes \mathbb{Z}_{2}^{\wedge} \cong\left(K_{1}(\mathbb{Z}) \star K_{i-1}(\mathbb{Z})\right) \otimes \mathbb{Z}_{2}^{\wedge},
$$


for $i \equiv 2 \bmod 8$, which is useful in order to calculate the 2 -adic product in the three remaining cases of the above list (see [14, Theorems 5.6, 5.7, 5.8 and 5.9] for the details of all these computations).

Finally, the fibration

$$
S U_{2}^{\wedge} \stackrel{\lambda}{\longrightarrow}\left(B G L(\mathbb{Z})^{+}\right)_{2}^{\wedge} \stackrel{\theta}{\longrightarrow} B O_{2}^{\wedge}
$$

also provides the determination of the Hopf algebra structure of the $\bmod 2$ cohomology of the infinite general linear group $G L(\mathbb{Z})$ as a module over the Steenrod algebra.

Theorem 9.28. There is an isomorphism of Hopf algebras and of modules over the Steenrod algebra

$$
\begin{aligned}
H^{*}(G L(\mathbb{Z}) ; \mathbb{Z} / 2) & \cong H^{*}\left(B G L(\mathbb{Z})^{+} ; \mathbb{Z} / 2\right) \\
& \cong H^{*}(B O ; \mathbb{Z} / 2) \otimes H^{*}(S U ; \mathbb{Z} / 2) \\
& \cong \mathbb{Z} / 2\left[w_{1}, w_{2}, w_{3}, \ldots\right] \otimes \Lambda_{\mathbb{Z} / 2}\left(u_{3}, u_{5}, u_{7}, \ldots\right),
\end{aligned}
$$

where the $w_{j}$ 's are the Stiefel-Whitney classes of degree $j(j \geq 1)$ and the classes $u_{2 k-1}$ are exterior classes of degree $2 k-1(k \geq 2)$.

Proof: The recent paper [15] contains the proof of this assertion (see Theorem 1 of [15]) and an explicit definition of the exterior classes $u_{2 k-1} \in$ $H^{2 k-1}\left(B G L(\mathbb{Z})^{+} ; \mathbb{Z} / 2\right)$ (see $[\mathbf{1 5}$, Definition 10 and Remark 14]). The classes $w_{j}$ are the images under $\theta^{*}: H^{*}(B O ; \mathbb{Z} / 2) \rightarrow H^{*}\left(B G L(\mathbb{Z})^{+} ; \mathbb{Z} / 2\right)$ of the universal Stiefel-Whitney classes in $H^{*}(B O ; \mathbb{Z} / 2)$ (see $[\mathbf{6 6}$, Chapter 7$])$. Notice that an additive version of the above isomorphism has been conjectured in [42, Corollary 4.3], and that the statement of the theorem can also be deduced from Theorem 4.3 and Remark 4.5 of [68] together with Corollary 9.20.

All these results provide a very deep knowledge of the homotopy type of the $K$-theory space $B G L(\mathbb{Z})^{+}$at the prime 2 . At odd primes, the situation is more difficult. If we would like to understand $B G L(\mathbb{Z})^{+}$at an odd prime $l$, we still have Bökstedt's space $J K(\mathbb{Z}, p)$ for all primes $p$. It is even possible to prove that if $p$ is well chosen, i.e., if $p$ generates the multiplicative group $\left(\mathbb{Z} / l^{2}\right)^{*}$, then the $l$-completion $J K(\mathbb{Z}, p)_{\hat{\ell}}$ of $J K(\mathbb{Z}, p)$ does not depend on $p$ (see [21, Proposition 3.24]): we shall denote it by $J K \mathbb{Z}_{\ell}^{\wedge}$. 
Definition 9.29. An odd prime $l$ is called a Vandiver prime if it does not divide the class number of the maximal real subfield of the cyclotomic field $\mathbb{Q}\left(\xi_{l}\right)$, where $\xi_{l}$ denotes an $l$-th primitive root of unity. It is known that all odd primes $\leq 4^{\prime} 000^{\prime} 000$ are Vandiver primes and it is a conjecture that all odd primes are Vandiver primes (see [98, Section 8.3]).

Theorem 9.30. For any Vandiver prime l, the space $J K \mathbb{Z}_{\hat{\ell}}^{\wedge}$ is a direct factor of $\left(B G L(\mathbb{Z})^{+}\right)_{\ell}$.

Proof: This theorem is due to C. Ausoni (see [21, Théorème 3.47]) and its proof is based on the result by W. G. Dwyer and S. A. Mitchell which asserts that $(U / O)_{\ell}^{\wedge}$ is a retract of $\left(B G L\left(\mathbb{Z}\left[\frac{1}{l}\right]\right)^{+}\right)_{\ell}^{\wedge}$ for Vandiver primes $l$ (see [43, Example 12.2]).

Again, it is easy to compute the homotopy groups of $J K \mathbb{Z}_{\ell}^{\wedge}$ : they contain the elements of infinite order given by Theorem 9.1 and the torsion classes detected by D. Quillen using the $K$-theory of finite fields (see Theorem 9.10).

Remark 9.31. If $l$ is a regular prime, the Lichtenbaum-Quillen conjecture (see Remark 9.16) is equivalent to the conjecture saying that there is a homotopy equivalence $\left(B G L(\mathbb{Z})^{+}\right)_{\ell} \simeq J K \mathbb{Z}_{\ell}$. However, at irregular primes, this cannot be true since the even dimensional homotopy groups

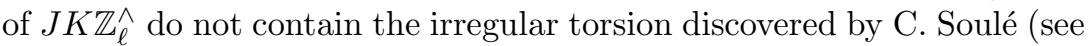
Theorem 9.13).

Nevertheless, Theorem 9.30 helps us to understand the homotopy type of $B G L(\mathbb{Z})^{+}$since it implies the three following results (see $[\mathbf{2 1}$, Théorèmes 4.17 and 5.14 and Proposition 5.18]).

Corollary 9.32. For any integer $i \equiv 1 \bmod 4(i \geq 5)$, the l-primary part $\left(\mu_{i}\right)_{l}$ of the integer $\mu_{i}$ (see Corollary 9.22) which describes the effect of the Hurewicz homomorphism on the elements of infinite order $b_{i} \in$ $K_{i}(\mathbb{Z})$ has the following property: if $l$ is a Vandiver prime, then $\left(\mu_{i}\right)_{l}=$ $\left(\left(\frac{i-1}{2}\right) !\right)_{l}$.

Corollary 9.33. For any integer $i \equiv 1 \bmod 4(i \geq 5)$, the l-primary part $\left(\rho_{i}\right)_{l}$ of the order $\rho_{i}$ of the $k$-invariant $k^{i+1}\left(B G L(\mathbb{Z})^{+}\right)$satisfies: if $l$ is a Vandiver prime, then $\left(\rho_{i}\right)_{l} \geq\left(\left(\frac{i-1}{2}\right) !\right)_{l}$. 
Corollary 9.34. If $l$ is a regular prime and if the Lichtenbaum-Quillen conjecture is true at $l$ (see Remark 9.31), then the integers $\left(\rho_{i}\right)_{l}$ can be exactly determined:

$$
\left(\rho_{i}\right)_{l}= \begin{cases}\left(\left(\frac{i-1}{2}\right) !\right)_{l}, & \text { if } i \equiv 1 \quad \bmod 4 \\ l(i+1)_{l}, & \text { if } 2(l-1) \text { is a proper divisor of }(i+1) \\ 1, & \text { otherwise }\end{cases}
$$

except for $\left(\rho_{11}\right)_{3}$ which is equal to 3 .

\section{Further developments}

The goal of this paper was to show how some topological methods can provide very general and deep results on the algebraic $K$-theory of rings. We especially emphasized the use of the infinite loop space structure of the $K$-theory space $B G L(R)^{+}$of any ring $R$, of cohomological calculations for linear groups, of the relationships between $K$-theory and linear group homology, and of the study of homotopical approximations. Of course, the arguments presented here do not represent all the topological considerations which can give interesting $K$-theoretical information.

It is not our purpose to describe these other ideas in details in this paper, but we just want to mention some of them. W. Browder applied in [36] the techniques of homotopy theory with finite coefficients (see $[69]$ ) in order to investigate the algebraic $K$-groups with coefficients in $\mathbb{F}_{p}$ for any prime $p$ and to deduce nice theorems on the ordinary algebraic $K$-theory: in particular, he could exhibit a periodicity result for the groups $K_{i}(\mathbb{Z})$ (see Theorem 9.10, Theorem 9.18 and Remark 9.19). F. Waldhausen introduced the $S$-construction which enabled him to present $K$-theoretical notions and results in a very general way over suitable categories (see for instance [97, Section 1.3], and [62]). W. G. Dwyer and E. M. Friedlander constructed in [41] another spectrum associated with a ring $R$, the étale $K$-theory spectrum of $R$, whose homotopy groups are called the étale $K$-groups of $R$ : they are in principle easier to calculate and some strong results are known on their relationships with the ordinary algebraic $K$-groups of $R$; however, the homomorphism relating these two $K$-theories is still the object of several difficult conjectures (see $[\mathbf{4 1}]$ and $[\mathbf{4 2}]$ for example). Some other impressive progress has been made by using techniques from stable homotopy theory (see for instance the works by M. Bökstedt, W. G. Dwyer, R. McCarthy, S. A. Mitchell, J. Rognes and C. Weibel). 
Let us finally recall that the definition of the algebraic $K$-theory of a $\operatorname{ring} R$ is based on the $Q$-construction over the category $\mathcal{P}(R)$ of finitely generated projective $R$-modules (see Remark 3.16): of course, this can also be done if we replace the category of finitely generated projective modules over a ring by another nice category. This shows that the main specificity of algebraic $K$-theory comes from its interaction with other areas of mathematics. Therefore, many $K$-theoretical results are consequences of methods from algebraic geometry, arithmetic, number theory, algebra, cyclic homology, operator theory, and so on. This explains also why almost all problems in algebraic $K$-theory are quite complicated and why many conjectures are still unsolved. However, this is obviously a sign of the great role that algebraic $K$-theory will play in the future.

\section{References}

[1] J. F. Adams, Vector fields on spheres, Ann. of Math. (2) 75 (1962), 603-632.

[2] J. F. AdAms, "Stable homotopy and generalised homology", Chicago Lectures in Mathematics, University of Chicago Press, Chicago, Ill., 1995, Reprint of the 1974 original.

[3] J. F. Adams, "Infinite loop spaces", Annals of Mathematics Studies 90, Princeton University Press, Princeton, N.J., 1978.

[4] D. Arlettaz, Chern-Klassen von ganzzahligen und rationalen Darstellungen diskreter Gruppen, Math. Z. 187(1) (1984), 49-60.

[5] D. Arlettaz, On the homology of the special linear group over a number field, Comment. Math. Helv. 61(4) (1986), 556-564.

[6] D. Arlettaz, On the $k$-invariants of iterated loop spaces, Proc. Roy. Soc. Edinburgh Sect. A 110(3-4) (1988), 343-350.

[7] D. Arlettaz, On the algebraic $K$-theory of $\mathbb{Z}, J$. Pure Appl. Algebra 51(1-2) (1988), 53-64.

[8] D. Arlettaz, The first $k$-invariant of a double loop space is trivial, Arch. Math. (Basel) 54(1) (1990), 84-92.

[9] D. Arlettaz, The Hurewicz homomorphism in algebraic $K$-theory, J. Pure Appl. Algebra 71(1) (1991), 1-12.

[10] D. Arlettaz, Universal bounds for the exponent of stable homotopy groups, Topology Appl. 38(3) (1991), 255-261.

[11] D. Arlettaz, A splitting result for the second homology group of the general linear group, in: "Adams Memorial Symposium on Algebraic Topology", volume 1 (Manchester, 1990), London Math. Soc. Lecture Note Ser. 175, Cambridge Univ. Press, Cambridge, 1992, pp. 83-88. 
[12] D. Arlettaz, The exponent of the homotopy groups of Moore spectra and the stable Hurewicz homomorphism, Canad. J. Math. 48(3) (1996), 483-495.

[13] D. Arlettaz and G. Banaszak, On the non-torsion elements in the algebraic $K$-theory of rings of integers, J. Reine Angew. Math. 461 (1995), 63-79.

[14] D. Arlettaz, G. Banaszak and W. Gajda, On products in algebraic K-theory, Comment. Math. Helv. 74(3) (1999), 476-506.

[15] D. Arlettaz, M. Mimura, K. Nakahata and N. Yagita, The mod 2 cohomology of the linear groups over the ring of integers, Proc. Amer. Math. Soc. 127(8) (1999), 2199-2212.

[16] D. Arlettaz and N. Pointet-Tischler, Postnikov invariants of $H$-spaces, Fund. Math. 161(1-2) (1999), 17-35.

[17] D. Arlettaz And P. Zelewski, Divisible homology classes in the special linear group of a number field, J. Pure Appl. Algebra 109(3) (1996), 221-229.

[18] D. Arlettaz And P. Zelewski, Linear group homology properties of the inclusion of a ring of integers into a number field, in: "Algebraic topology: new trends in localization and periodicity" (Sant Feliu de Guíxols, 1994), Progr. Math. 136, Birkhäuser, Basel, 1996, pp. 23-31.

[19] M. F. Aтіуан, "K-theory", second ed., Advanced Book Classics, Addison-Wesley Publishing Company Advanced Book Program, Redwood City, CA, 1989, Notes by D. W. Anderson.

[20] M. F. ATIYAh And F. HiRzebruch, Riemann-Roch theorems for differentiable manifolds, Bull. Amer. Math. Soc. 65 (1959), 276-281.

[21] C. Ausoni, Propriétés homotopiques de la $K$-théorie algébrique des entiers, Ph.D. thesis, Université de Lausanne (1998).

[22] A. BAK, Editorial, K-Theory 1(1) (1987), 1-4.

[23] G. Banaszak, Algebraic $K$-theory of number fields and rings of integers and the Stickelberger ideal, Ann. of Math. (2) 135(2) (1992), 325-360.

[24] G. Banaszak, Generalization of the Moore exact sequence and the wild kernel for higher $K$-groups, Compositio Math. 86(3) (1993), 281-305.

[25] G. Banaszak and P. Zelewski, Continuous $K$-theory, $K$-Theory 9(4) (1995), 379-393.

[26] M. BarratT And S. Priddy, On the homology of non-connected monoids and their associated groups, Comment. Math. Helv. 47 (1972), 1-14. 
[27] H. BAss, "Algebraic K-theory", W. A. Benjamin, Inc., New YorkAmsterdam, 1968.

[28] G. Baumslag, E. Dyer and A. Heller, The topology of discrete groups, J. Pure Appl. Algebra 16(1) (1980), 1-47.

[29] A. J. BeRRICK, "An approach to algebraic K-theory", Research Notes in Mathematics 56, Pitman (Advanced Publishing Program), Boston, Mass., 1982.

[30] M. BökstedT, The rational homotopy type of $\Omega \mathrm{Wh}^{\mathrm{Diff}}(*)$, in: "Algebraic topology" (Aarhus, 1982), Lecture Notes in Math. 1051, Springer, Berlin, 1984, pp. 25-37.

[31] A. Borel, Cohomologie réelle stable de groupes $S$-arithmétiques classiques, C. R. Acad. Sci. Paris Sér. A-B 274 (1972), A1700-A1702.

[32] A. Borel, Stable real cohomology of arithmetic groups, Ann. Sci. École Norm. Sup. (4) 7 (1974), 235-272.

[33] A. Borel AND J.-P. SERre, Le théorème de Riemann-Roch (d'après des résultats inédits d'A. Grothendieck), Bull. Soc. Math. France 86 (1958), 97-136.

[34] S. BorewiCz AND I. ŠAfArevič, "Zahlentheorie", Mathematische Reihe 32, Birkhäuser Verlag, Basel-Stuttgart, 1966.

[35] R. Bотт, The stable homotopy of the classical groups, Ann. of Math. (2) 70 (1959), 313-337.

[36] W. Browder, Algebraic $K$-theory with coefficients $\mathbb{Z} / p$, in: "Geometric applications of homotopy theory I" (Proc. Conf., Evanston, Ill., 1977), Lecture Notes in Math. 657, Springer, Berlin, 1978, pp. $40-84$.

[37] K. S. Brown, "Cohomology of groups", Graduate Texts in Mathematics 87, Springer-Verlag, New York, 1982.

[38] H. Cartan, Algèbres d'Eilenberg-Maclane et homotopie, Sém. H. Cartan Ecole Norm. Sup., exposé 11 (1954/55).

[39] A. Dold And R. Thom, Quasifaserungen und unendliche symmetrische Produkte, Ann. of Math. (2) 67 (1958), 239-281.

[40] A. Douady, Périodicié du groupe unitaire, Sém. H. Cartan Ecole Norm. Sup., exposé 11 (1959/60).

[41] W. G. Dwyer And E. M. Friedlander, Algebraic and étale K-theory, Trans. Amer. Math. Soc. 292(1) (1985), 247-280.

[42] W. G. DWyer And E. M. Friedlander, Conjectural calculations of general linear group homology, in: "Applications of algebraic K-theory to algebraic geometry and number theory", Part I, 
II (Boulder, Colo., 1983), Contemp. Math. 55, Amer. Math. Soc., Providence, R.I., 1986, pp. 135-147.

[43] W. G. Dwyer And S. A. Mitchell, On the $K$-theory spectrum of a ring of algebraic integers, K-Theory 14(3) (1998), 201-263.

[44] Z. Fiedorowicz AND S. Priddy, "Homology of classical groups over finite fields and their associated infinite loop spaces", Lecture Notes in Mathematics 674, Springer, Berlin, 1978.

[45] S. M. Gersten, On the spectrum of algebraic $K$-theory, Bull. Amer. Math. Soc. 78 (1972), 216-219.

[46] C. H. Giffen, Bott periodicity and the $Q$-construction, in: " $A l$ gebraic K-theory" (Poznań, 1995), Contemp. Math. 199, Amer. Math. Soc., Providence, RI, 1996, pp. 107-124.

[47] B. Gray, "Homotopy theory", An introduction to algebraic topology, Pure and Applied Mathematics 64, Academic Press [Harcourt Brace Jovanovich Publishers], New York, 1975.

[48] J.-C. Hausmann and D. Husemoller, Acyclic maps, Enseign. Math. (2) 25(1-2) (1979), 53-75.

[49] A. Heller, On the homotopy theory of topogenic groups and groupoids, Illinois J. Math. 24(4) (1980), 576-605.

[50] P. J. Hilton and U. Stammbach, "A course in homological algebra", second ed., Graduate Texts in Mathematics 4, SpringerVerlag, New York, 1997.

[51] D. Husemoller, "Fibre bundles", second ed., Graduate Texts in Mathematics 20, Springer-Verlag, New York, 1975.

[52] D. M. Kan And W. P. Thurston, Every connected space has the homology of a $K(\pi, 1)$, Topology 15(3) (1976), 253-258.

[53] M. Karoubi and O. Villamayor, $K$-théorie algébrique et K-théorie topologique, I, Math. Scand. 28 (1971), 265-307.

[54] M. A. Kervaire, Multiplicateurs de Schur et $K$-théorie, in: "Essays on Topology and Related Topics (Mémoires dédiés à Georges de Rham)", Springer, New York, 1970, pp. 212-225.

[55] R. Lee and R. H. Szczarba, The group $K_{3}(\mathbb{Z})$ is cyclic of order forty-eight, Ann. of Math. (2) 104(1) (1976), 31-60.

[56] R. Lee And R. H. Szczarba, On the torsion in $K_{4}(\mathbb{Z})$ and $K_{5}(\mathbb{Z})$, Duke Math. J. 45(1) (1978), 101-129.

[57] S. Lichtenbaum, Values of zeta-functions, étale cohomology, and algebraic $K$-theory, in: "Algebraic $K$-theory, II: "Classical" algebraic K-theory and connections with arithmetic" (Proc. Conf., Battelle Memorial Inst., Seattle, Wash., 1972), Lecture Notes in Math. 342, Springer, Berlin, 1973, pp. 489-501. 
[58] E. Lluis-Puebla, J.-L. Loday, H. Gillet, C. Soulé and V. Snaith, "Higher algebraic K-theory: an overview", Lecture Notes in Mathematics 1491, Springer-Verlag, Berlin, 1992.

[59] J.-L. LODAY, $K$-théorie algébrique et représentations de groupes, Ann. Sci. École Norm. Sup. (4) 9(3) (1976), 309-377.

[60] S. Mac Lane, Homologie des anneaux et des modules, in: "Colloque de topologie algébrique, Louvain, 1956", Georges Thone, Liège, 1957, pp. 55-80.

[61] C. R. F. MAUnder, A short proof of a theorem of Kan and Thurston, Bull. London Math. Soc. 13(4) (1981), 325-327.

[62] R. McCarthy, On fundamental theorems of algebraic $K$-theory, Topology 32(2) (1993), 325-328.

[63] R. MCCARTHY, A chain complex for the spectrum homology of the algebraic $K$-theory of an exact category, in: "Algebraic K-theory" (Toronto, ON, 1996), Fields Inst. Commun. 16, Amer. Math. Soc., Providence, RI, 1997, pp. 199-220.

[64] J. Milnor, Algebraic $K$-theory and quadratic forms, Invent. Math. 9 (1969/1970), 318-344.

[65] J. Milnor, "Introduction to algebraic K-theory", Annals of Mathematics Studies 72, Princeton University Press, Princeton, N.J., 1971.

[66] J. W. Milnor and J. D. Stasheff, "Characteristic classes", Annals of Mathematics Studies 76, Princeton University Press, Princeton, N. J., 1974.

[67] S. A. Mitchell, The Morava $K$-theory of algebraic $K$-theory spectra, $K$-Theory 3(6) (1990), 607-626.

[68] S. A. Mitchell, On the plus construction for $B G L \mathbb{Z}\left[\frac{1}{2}\right]$ at the prime 2, Math. Z. 209(2) (1992), 205-222.

[69] J. Neisendorfer, Primary homotopy theory, Mem. Amer. Math. Soc. 25(232) (1980).

[70] D. Quillen, Cohomology of groups, in: "Actes du Congrès International des Mathématiciens" (Nice, 1970), Tome 2, GauthierVillars, Paris, 1971, pp. 47-51.

[71] D. Quillen, On the cohomology and $K$-theory of the general linear groups over a finite field, Ann. of Math. (2) 96 (1972), 552-586.

[72] D. Quillen, Higher algebraic $K$-theory, I, in: "Algebraic $K$-theory, I: Higher K-theories" (Proc. Conf., Battelle Memorial Inst., Seattle, Wash., 1972), Lecture Notes in Math. 341, Springer, Berlin, 1973, pp. 85-147.

[73] D. Quillen, Finite generation of the groups $K_{i}$ of rings of algebraic integers, in: "Algebraic K-theory, I: Higher K-theories" 
(Proc. Conf., Battelle Memorial Inst., Seattle, Wash., 1972), Lecture Notes in Math. 341, Springer, Berlin, 1973, pp. 179-198.

[74] D. Quillen, Higher $K$-theory for categories with exact sequences, in: "New developments in topology" (Proc. Sympos. Algebraic Topology, Oxford, 1972), London Math. Soc. Lecture Note Ser. 11, Cambridge Univ. Press, London, 1974, pp. 95-103.

[75] D. Quillen, Letter from Quillen to Milnor on $\operatorname{Im}\left(\pi_{i} O \rightarrow \pi_{i}^{s} \rightarrow\right.$ $K_{i} \mathbb{Z}$ ), in: "Algebraic K-theory" (Proc. Conf., Northwestern Univ., Evanston, Ill., 1976), Lecture Notes in Math. 551, Springer, Berlin, 1976, pp. $182-188$.

[76] J. Rognes, $K_{4}(\mathbb{Z})$ is the trivial group, Topology 39(2) (2000), $267-281$.

[77] J. Rognes And C. Weibel, Two-primary algebraic $K$-theory of rings of integers in number fields, J. Amer. Math. Soc. 13(1) (2000), 1-54.

[78] J. RosenberG, "Algebraic K-theory and its applications", Graduate Texts in Mathematics 147, Springer-Verlag, New York, 1994.

[79] J. J. Rotman, "An introduction to algebraic topology", Graduate Texts in Mathematics 119, Springer-Verlag, New York, 1988.

[80] C.-H. SAH, Homology of classical Lie groups made discrete, III, J. Pure Appl. Algebra 56(3) (1989), 269-312.

[81] J. ScherER, Exponents for high-dimensional gamma groups, Exposition. Math. 13(5) (1995), 455-468.

[82] J.-P. SERRE, Groupes d'homotopie et classes de groupes abéliens, Ann. of Math. (2) 58 (1953), 258-294.

[83] J. R. Silvester, "Introduction to algebraic K-theory", Chapman and Hall Mathematics Series, Chapman \& Hall, London, 1981.

[84] C. Soulé, Addendum to the article: "On the torsion in $K_{*}(\mathbb{Z})$ ", Duke Math. J. 45(1) (1978), 131-132.

[85] C. Soulé, $K$-théorie des anneaux d'entiers de corps de nombres et cohomologie étale, Invent. Math. 55(3) (1979), 251-295.

[86] C. Soulé, Groupes de Chow et $K$-théorie de variétés sur un corps fini, Math. Ann. 268(3) (1984), 317-345.

[87] C. Soulé, Opérations en K-théorie algébrique, Canad. J. Math. 37(3) (1985), 488-550.

[88] C. Soulé, On the 3-torsion in $K_{4}(\mathbb{Z})$, Topology 39(2) (2000), 259-265.

[89] E. H. Spanier, "Algebraic topology", McGraw-Hill Book Co., New York, 1966.

[90] V. SRInivas, "Algebraic K-theory", second ed., Progress in Mathematics 90, Birkhäuser Boston Inc., Boston, MA, 1996. 
[91] A. A. SusLin, Homology of $G L_{n}$, characteristic classes and Milnor $K$-theory, in: "Algebraic K-theory, number theory, geometry and analysis" (Bielefeld, 1982), Lecture Notes in Math. 1046, Springer, Berlin, 1984, pp. 357-375.

[92] A. A. Suslin, Algebraic K-theory of fields, in. "Proceedings of the International Congress of Mathematicians", Vol. 1 (Berkeley, Calif., 1986) (Providence, RI), Amer. Math. Soc., 1987, pp. 222-244.

[93] R. G. SWAn, Vector bundles and projective modules, Trans. Amer. Math. Soc. 105 (1962), 264-277.

[94] R. M. Switzer, "Algebraic topology - homotopy and homology”, Die Grundlehren der mathematischen Wissenschaften 212, Springer-Verlag, New York, 1975.

[95] V. Voevodsky, The Milnor conjecture, Preprint (1996).

[96] J. B. WAGONER, Delooping classifying spaces in algebraic $K$-theory, Topology 11 (1972), 349-370.

[97] F. Waldhausen, Algebraic $K$-theory of spaces, in: "Algebraic and geometric topology" (New Brunswick, N.J., 1983), Lecture Notes in Math. 1126, Springer, Berlin, 1985, pp. 318-419.

[98] L. C. Washington, "Introduction to cyclotomic fields", second ed., Graduate Texts in Mathematics 83, Springer-Verlag, New York, 1997.

[99] C. Weibel, The 2-torsion in the $K$-theory of the integers, $C . R$. Acad. Sci. Paris Sér. I Math. 324(6) (1997), 615-620.

[100] G. W. Whitehead, "Elements of homotopy theory", Graduate Texts in Mathematics 61, Springer-Verlag, New York, 1978.

[101] J. H. C. Whitehead, The homotopy type of a special kind of polyhedron, Ann. Soc. Polon. Math. 21 (1948), 176-186.

[102] J. H. C. Whitehead, A certain exact sequence, Ann. of Math. (2) 52 (1950), 51-110.

Institut de Mathématiques

Université de Lausanne

CH-1015 Lausanne

Switzerland

E-mail address: dominique.arlettaz@ima.unil.ch

Primera versió rebuda el 30 d'abril de 1999, darrera versió rebuda el 6 de març de 2000. 